
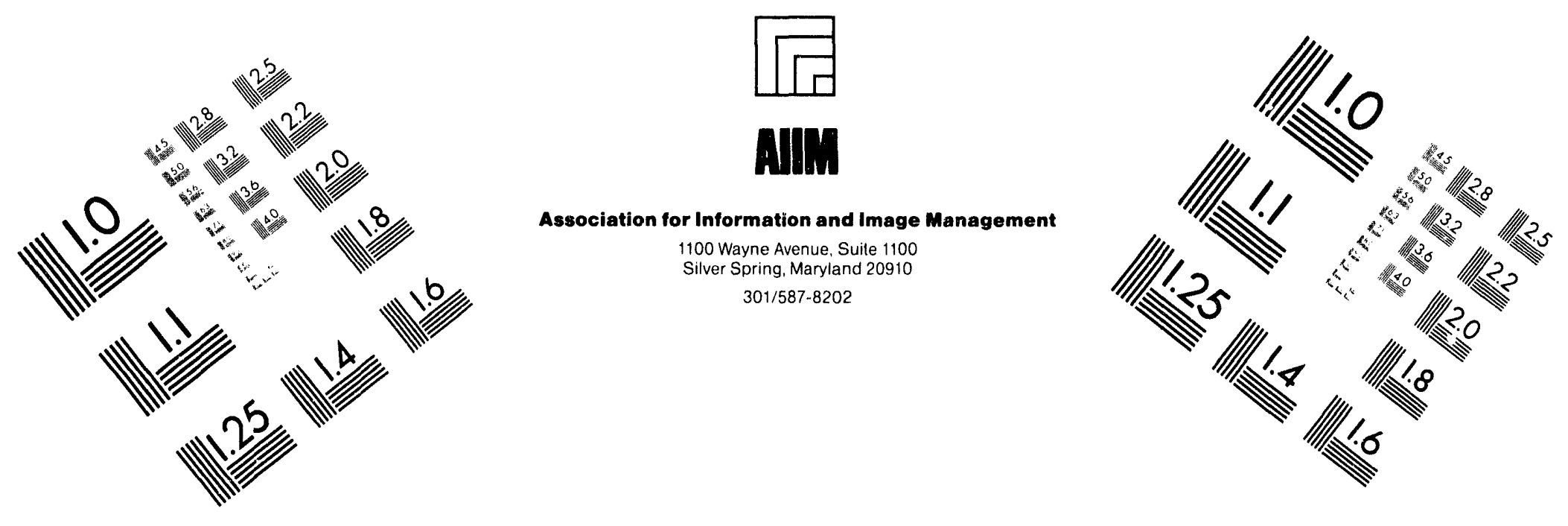

\title{
Centimeter
}

| Inches
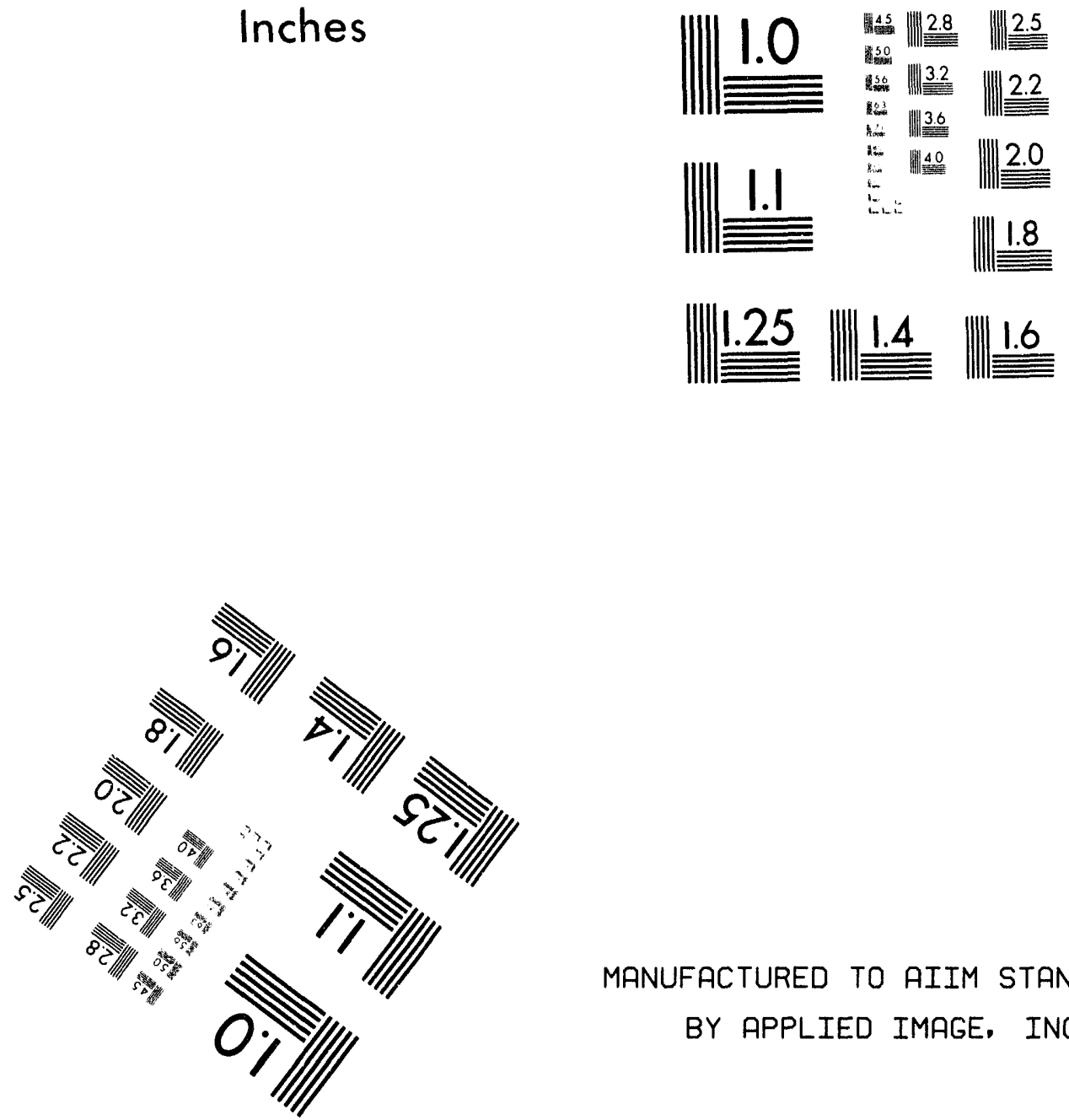

MANUFACTURED TO AIIM STANDARDS

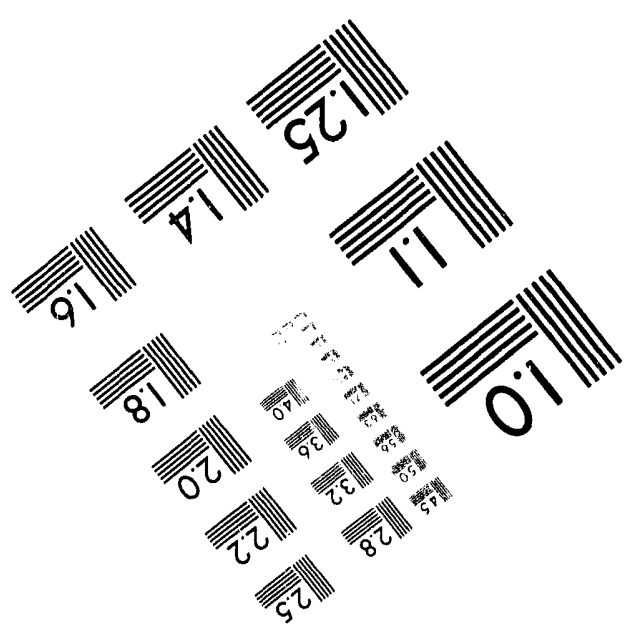



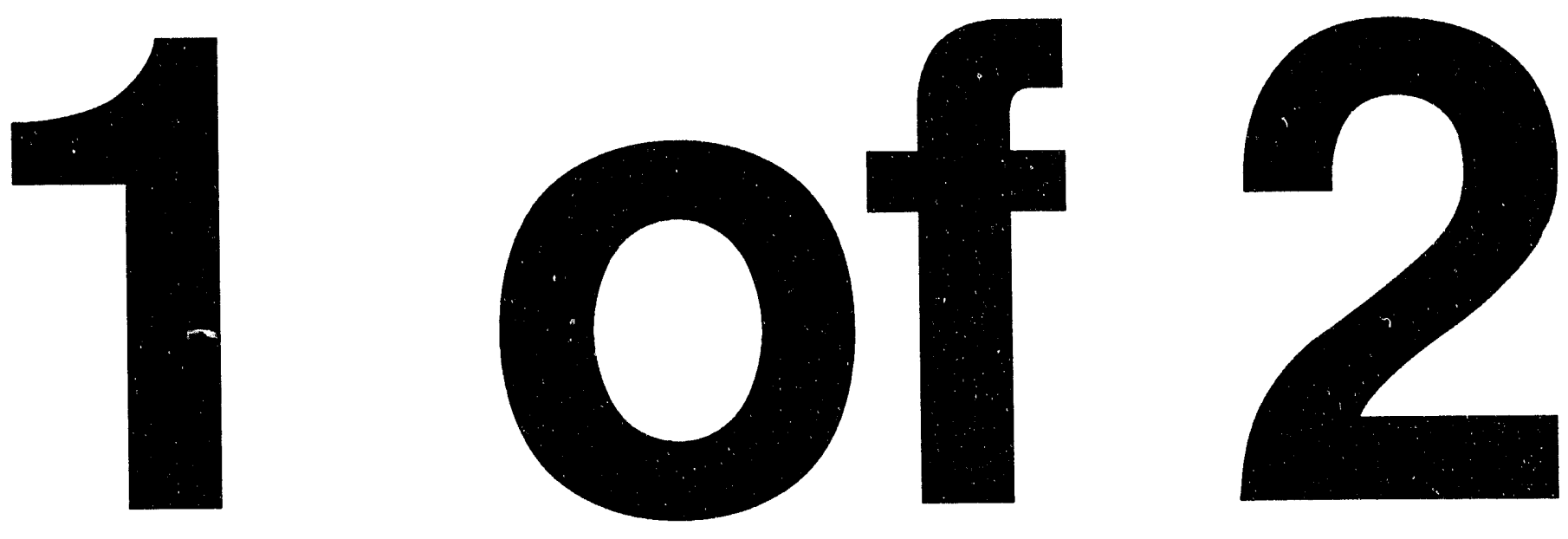


$$
\mid A-54 B B=94-81
$$

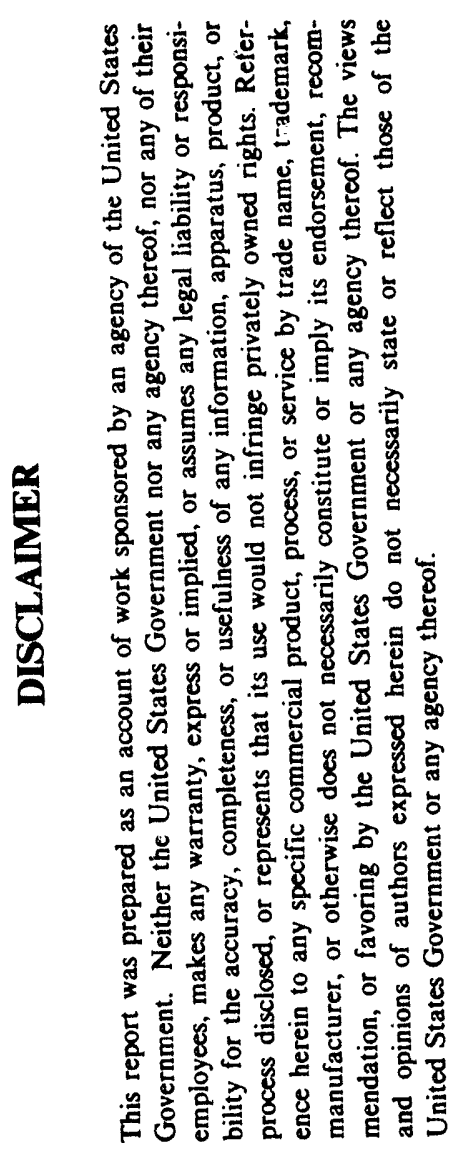

THE BEAR PROGRAM NL PLASMA PHYSICS INSTRUMENTATION MEASUREMENTS

NAVAL RESEARCH LABORATORY WASHINGTON, DC 20375-5000

15 NOVEMBER 1989

Said h Wall

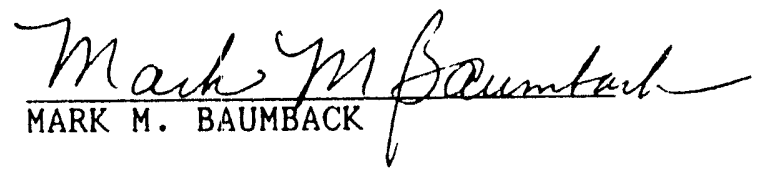

Daniel A. That
Paul Radius

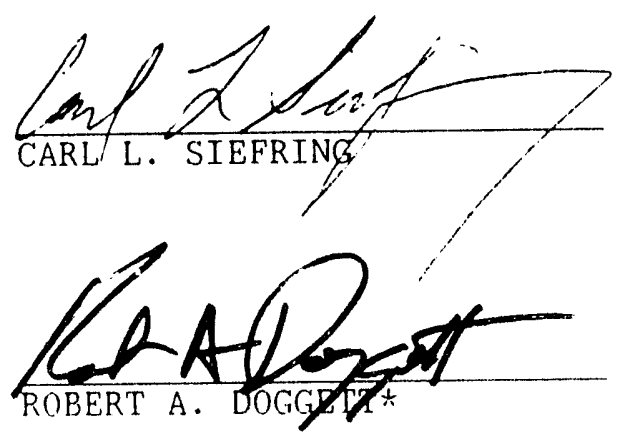

*SACHS FREEMAN

MASTER

DISTREYUTION OF THIS DOCUMENT IS UNLMMTED 
THE BEAR PROGRAM

NRL PLASMA PHYSICS INSTRUMENTATION MEASUREMENTS

TABLE OF CONTENTS

I. Introduction

II. Langmuir Probes

II.1 Design Considerations

II.2 Instrument Development

II.3 Bench Tests \& Calibration

II.4 Environmental Testing

II.5 Integration

II.6 Flight Performance Data

III. High Voltage Device

III.1 Design Consideratior:s

III.2 Instrument Development

III.3 Bench Tests \& Calibration

III.4 Environmental Testing

III.5 Integration

III.6 Flight Performance Data

IV. Plasma Wave Receiver

IV.1 Design Considerations

IV.2 Instrument Development

IV. 3 Bench Tests \& Calibration

IV.4 Environmental Testing

IV. 5 Integration

IV.6 Flight Performance Data

V. Appendix 
I. Introduction

This report summarizes U.S. Naval Research Laboratory (NRL), Plasma Physics Division, contributions to an instrument complement flown aboard an ARIES rocket as part of the Los Alamos (LANL) Beam Experiments Aboard a Rocket (BEAR) program.

The BEAR program was a joint effort to launch, and demonstrate the feasibility of operating, a $1 \mathrm{MeV} 10 \mathrm{ma}$ Neutral Particle Beam (NPB) accelerator from a space platform. The accelerator design and manufacture were the responsibility of Los Alamos National Lab (LANL); diagnostics associated with accelerator operation and beam-plasma effects were also to be undertaken by LANL and NRL. Payload Integration and Telemetry was provided by the Air Force Geophysical Lab (AFGL) and Northeastern University (NEU). Beam effects on the local plasma in addition to accelerator produced vehicle effects (e.g., charging) were the responsibility of NRL as outlined herein. The program was managed and funded by the SDIO office, Washington, D.C.

The BEAR rocket was launched successfully during the early morning hours of July 13 from White Sands Missile Range, White Sands, N.M. The NRL contribution to this effort included three instrument packages designed to diagnose beam-plasma and vehicle-plasma interactions. The instruments included:

(1) A Langmuir probe (LP) degign consisting of 4 separate sensors. The instrument measures electron density, temperature and vehicle charging as described in this report.

(2) A HIgh Voltage (HIV) Langmuir Probe designed to monitor vehicle charging through current polarity changes and,

(3) A Plasma Wave Receive (PWR) designed to characterize the plasma wave emissions covering a broad frequency range from near DC to $50 \mathrm{MHz}$.

This report is submitted in fulfillment of an agreement between LANL and NRL. It covers each instrument in detail in addition to data analysis to the present date. 
II.1 Langmuir Probes Design Considerations

\section{Instrument Description}

The Langmuir probe measures electron density, temperature, and power spectra derived from variations in electron density, which are associated with electrostatic oscillations in the local plasma. In addition, it measures vehicle potential within specified ranges according to design.

The sensors, which are typically employed in pairs, are cylindricul Langmuir probes separated from the vehicle by deployable booms. When the probes are swept several volts positive and negative with respect to the vehicle, a varying current is drawn from the plasma. From the current-voltage relationship, the plasma characteristics mentioned above are derived under the assumption that the plasma distribution function derives from MaxwellBoltzmann statistics. Vehicle potential can be measured if it falls within the range of probe sweep voltage.

Spatially separated sensors can also measure the phase velocity of propagating electrostatic waves using cross-correlation techniques if appropriate interferometric schemes are employed(1). The frequency range is primarily limited by the available telemetry space. In previous applications Nyquist frequencies have ranged from $0.25 \mathrm{KHz}$ to $4 \mathrm{KHz}$.

In the BEAR configuration four probes were deployed from the Beam Diagnostics Section as seen in Figure II.1. The probes were operated in pairs according to the sequencing scheme shown in Figure II.2. As configured each probe was deployed so that it had a free field of view to the beam axis. The four-probe sequencing allows simultaneous measurements at two separate radial positions in addition to alternately providing these measurements at fixed radial positions.

Each probe pair consists of a probe whose constant bias level is positive (E-probe) and one whose constant bias level is negative (I-probe). The E-probe then collects electron saturation current and the I-probe collects ion saturation current during application of the constant bias. Each probe is pulsed between an applied sweep and the constant bias level. Figure II. 3 shows the voltages applied to each probe.

Figure II. 4 shows logic cycling sequences of operation for each of the probes. Dotted lines in the probe outputs (horizontal) indicate that a particular probe is not being monitored. For example, during the first four segments (vertical sweep intervals separated by dotted lines) probe 1 is being monitored along with probe 2 (probe 1 operates as an E-probe, probe 2 as an I-probe). Probes 3 and 4 are not being monitored during this time.

\section{Operational Design Basis}

The basic operation characteristics of the pulsed Langmuir probes employed are well-documented in the literature (3) and will not be covered 
here. Of primary concern are the characteristics of the present configuration, as described above, and the basis for that particular design choice.

As is indicated in Figure II.2 probe operation is duty cycled among alternate probe pairs. Other than obtaining a more complete data set azimuthally, the primary reason was the possible occurrence of a beam-plasma instability which has been observed under similar conditions in both laboratory and space plasmas. $(1,2)$ The Beam Plasma Discharge(BPD) has been ignited during electron accelerator operition where beam energies are of the order of $\mathrm{keV}$ and currents tens of $\mathrm{Ma}$ or higher. Experience in laboratory environments and elsewhere indicates that during BPD a region of intense radiation emission and elevated density exists radially along the beam path. Inside this region which can be as wide as a meter or more, instrument saturation is likely. Hence, the positioning of probes (1) and (2) and probe sequencing, could avoid saturation in the event that BPD occurred. We desire, on the other hand, to sample the environment as close to the beam as possible; hence the probe positions (3) and (4). A related reason for placing probes at two different radial locations involves possible electrostatic wave generation which can occur as a result of instabilities generated during accelerator operation. Waves generated with a radial $k$ component could be detected crossing each probe location and characterized in phase velocity and wavelength under certain assumptions.

In addition to measurement of electrostatic wave phenomera, sampling of electron density both in the beam exit region and in the ambient medium was desirable. Since probes aligned axially would show large wake and magnetic field effects on the upleg before pitchover, and those at outer angled sites would not, this "two region" sampling was possible. The magnetic field aspect effect on the outer probes whose angle is near $30^{\circ}$ wrt $B$ is such that about $70 \%$ of maximum current is collected for these densities $\left(\mathrm{Ne} \sim 10^{3} \mathrm{~cm}^{-3}\right)$ according to theory (4). This was accomplished and the results are discussed in jection II.6.

\section{II.2 Instrument Development}

\section{Overview}

The basic NRL pulsed probe design was modified considerably for the BEAR program; another set of probes was added as noted abo\%e, a long pulsing interval with voltage levels varying from -30 to +30 volts iriven by gun firings was designed, and an outboard switching box (logic and circuitry) was completed during design modification. These changes are incorporated into the block diagram of Figure II.5 which shows the instrument at the board level. The relationship of these changes to the remainder of the instrument package is also discernible from this Figure. All probes and associated electronics hardware were mounted in the Beam Diagnostics (BD) section near beam exit as seen in Figure II.1. 


\section{Electronics-Microview}

The functioning of the E-probe electronics is based on two applied sweep periods (as seen in Figure II.3) followed by two periods at the fixed bias level (i.e., not pulsed). Therefore the time required for the sequence of two pulsed sweeps followed by the constant bias level is near $3.2 \mathrm{sec}$. Data was sampled at a $4 \mathrm{khz}$ rate in a sequence of three bias samples and one sweep sample during the pulsed sweep portion; during the period of constant bias the sampling rate remained at $4 \mathrm{khz}$ so that the Nyquist frequency is $2 \mathrm{khz}$ during this period. As mentioned, the addition of a second probe set required that an outboard switching box be added. Figures II. 4 and II.5 show the switching box timing, logic and relationship to downstream electronics. Circuit diagrams of the switching box are also included in the Appendix. From Figure II.4 it can be seen that there is no provision to synchronize the probe sweep either with a gun pulse or with the telemetry signal. The result of this is that sampling of probe currents occurs at different points in the gun period interval; chis allows a reconstruction of the pulse during data reduction as covered in Section II.6. Figures II.6 and II.7 show sweep and timing logic and their relationship to gun pulse timing.

Unlike the E-probe electronics, I-probe sweep voltage levels as seen in Figure II. 3 were driven by the gun pulse pre-fire signal. Figure II. 8 shows the logic sequence in which the synchronized stepped sweep alternates with a fixed baseline level. The switched Sample and Hold (S/H) network, which inserts data taken during a gun pulse into the normal $4 \mathrm{khz}$ data stream, is also included in this Figure.

The Gun Pulse Synchronized Sweep board of the I-probe can be broken down into several subsections for ease of discussion; the first of these subsections is the sweep generator. The sweep is triggered by the pre-fire pulse which precedes the $5 \mathrm{~Hz}$ gun pulse by $448 \mu \mathrm{s}$ (Figure II.9); each prefire pulse triggers a $100 \mathrm{mS}$ multivibrator and advances a binary up counter. The counter feeds a D/A converter which produces a 16 step signal (i.e., the sweep levels) which is sent to one pole of an SPDT switch; the other pole of the switch has a fixed voltage for the baseline (Figure II.8). The switch is controlled by the $100 \mathrm{mS}$ multivibrator and the $5 \mathrm{~Hz}$ rate of the pre-fire pulse. After the sweep amplifier the signal is converted to a lower voltage and used as a sweep monitor. It is important to note that without the prefire pulse there is no sweep.

The second subsection consists of the switched $S / H$ network and its associated timing multivibrators (See Figures II.5 and II.8). The I-probe samples once during the $50 \mathrm{\mu sec}$ pulse by design; this is differen, from the E-probe whose samples during gun pulse periods occur at different times due to a relative drift between the samples and the gun pulse The logical rule followed is that whenever the gun pulse occurs the data sampled will be sent to the telemeter during the next telemetry readout interval. Between gun pulses, the data from the amplifier board (where the range monitor latches have been tied open and the analog data sample and hold has been removed) is fed to another $\mathrm{S} / \mathrm{H}$ circuit that is gated by the $47 \mathrm{\mu s} 4 \mathrm{KHz}$ pulse from the Sweep and Logic board. This data is then sent through a switch and out to the telemeter. However, when a gun pulse occurs one input to an AND gate is set 
high. As the end of the next $4 \mathrm{KHz}$ sample occurs, the Sweep and Logic board generates an End of Sample pulse to the same AND gate. As the signal passes through the gate, it triggers a $200 \mu \mathrm{s}$ multivibrator the output of which switches the data stored in the gun S/H circuit to the telemeter. The falling edge of the $200 \mu$ s pulse triggers another multivibrator which resets the $R-S$ latch so that sweep data can now be fed through to the telemeter. Figure II. 8 shows the relationship between the gun pulse generated timing signals.

The third subsection generates the signals needed to control the electrometer switch position. Figure II.10 shows electrometer switching logic on the I-probe amplifier board that is needed to accommodate the signals generated by the Gun Pulse Synchronized Sweep board (See Figure II.8). At first, the pre-fire pulse triggers the $340 \mu \mathrm{s}$ multivibrator near the bottom of Figure II.8. The output then latches the electrometer switch not allowing it to change prior to a gun pulse. The falling edge of the $340 \mu \mathrm{s}$ pulse triggers a multivibrator that produces pulse $C$. This pulse gates the position stored in the gun pulse Schmitt circuit out to the type D latch, which sets the electrometer switch in its position before the last gun pulse; it also clocks the data through the latch. When the gun pulse occurs, pulse A gates the current electrometer switch position into the gun pulse Schmitt circuit. Pulse B is produced by the falling edge of the $47 \mu \mathrm{s}$ gun pulse S/H, triggering a multivibrator whose output generates a short pulse to an OR gate ; the falling edge also enables the AND gates to gate through pulses $B$ and $D$ for the non-gun pulse samples. Pulse B both gates the switch position stored in the non-gun pulse sample Schmitt circuit to the type D latch and also clocks it through the latch in a fashion similar to pulse C. Pulse D gates the current position of the electrometer switch to the Schmitt circuit during non-gun pulse samples.

\section{Flight Instrument Manufacture}

The PC board layouts were fabricated at NRL, boards were assembled and the instrument's electronics box was given a conductive coating. The probe booms were manufactured and gold plated at NRL facilities. Gold plating and constant cleansing of boom and sensor elements before launch assures a high conductivity and lessens the chance of charge buildup on dielectric surfaces which could distort the I-V characteristic response of the instrument.

\section{3 Bench Tests and Calibration}

Bench testing of the assembled instrument consists largely of adjustments made to differential amplifiers, switching rectifiers, amplifier stages and D/A converters. Connecting the probe sensor element through a series of resistors to ground and photographing oscilloscope outputs allows a determination of pre-calibration operation integrity with respect to range switching level matching and overlap,i.e., the instrument is configured to switch over 20 range levels as a function of the magnitude of collected current. Since these levels have an inhetent overlap, care must be taken to insure that the levels match to within strict tolerance levels. 
The probe calibration procedure takes place through a MICROVAX II based two stage A/D conversion routine which samples output currents (in the form of voltages) for given applied voltages and calculates calibration coefficients in the form,

$$
I=A_{i} V+B_{i}
$$

for each of 20 ranges $i$. The final stage of the calibration consists of plotting $I-V$ responses to applied voltages for each range $i$ for which $A_{1}, B_{1}$ have been determined. The coefficient calibrations for each of the probes is found in Tables II.1 and II.2 of the Appendix. A sample calibrated output is shown in Figure A.1 of the Appendix for a resistance of 10 megohms attached to the sensor element.

\section{II.4 Environmental Tests}

The instrument was subjected to the environmental tests required by the Bear Project office (BPO) in August of 1988.

\section{Shake and Vibration}

A $50 \mathrm{~g}$ peak half sine shock pulse lasting for $11 \mathrm{~ms}$ was applied to the instrument in each of three orthogonal axes for a total of six pulses. A random vibration spectrum was applied in each of the axes for one minute per axis. The specifications for the vibration spectrum were as follows:

$\begin{array}{ll}\text { Frequency }(\mathrm{hz}) & \text { Amplitude } \\ 20-100 & 6 \mathrm{db} / \text { octave } \\ 100-2000 & .042 \mathrm{~g} / \mathrm{hz} \\ \text { Overall } & 9 \mathrm{~g} \mathrm{rms}\end{array}$

\section{Thermal Cycling}

Testing was performed in a chamber in which the temperature was lowered to $-24 \mathrm{deg} C$ during a functional cycle. The chamber temperature is then recycled to $65 \mathrm{deg} \mathrm{C}$ again while the instrument operates. This procedure was repeated two more times for a total of three cycles.

\section{Vacuum Testing}


The electronics is finally placed in a vacuum chamber which operates near $.05 \mathrm{~mm} \mathrm{Hg}$. Operating at this level and during pump down confirms functioning in the low and intermediate pressure regimes.

Instrument stripouts during all of the functional testing outlined above is available upon request from NRL. Environmental testing sample waveforms of shock and random vibration applied to each instrument are shown in the Appendix along with thermal and vacuum specifications required by LANL.

\section{II.5 Integration}

The Langmuir probes were integrated a number of times into the telemetry stream during testing of the Telemetry Physics (TM Physics) payload at the Air Force Geophysical Laboratory (AFGL) beginning in March 1988 and extending through the year. Since the instrument (probes in addition to electronics) was not physically a part of this payload, it could only be tested as operational and non-interfering through the telemetry stream during the series of preliminary integration tests which were performed before mating to the accelerator and Beam Diagnostics Section (BD Section). Final

integration and environmental testing had to await this mechanical mating which took place at Los Alamos National Laboratory (LANL). Probe timed deployment, turn on sequences, and interfaces through the accelerator section to telemetry were all confirmed in a series of tests defined by LANL outlined elsewhere in this document. The pinouts and cable matings are listed in the Appendix.

\section{II.6 Flight Performance and Data}

The Langmuir probes performed as expected throughout the BEAR flight. Deployed as planned, beginning at $92 \mathrm{sec}$ (TALO), all four probes were deployed by $96 \mathrm{sec}$. There is a visible cinfirmation of this deployment by the television camera looking out the rear of the BD section. In addition to providing measurements of temperature and density for the entire flight, the probes are providing crucial information related to vehicle charging. By design, a number of probe samples were taken during the $50 \mu \mathrm{s}$ gun pulse and so we are able to unfold a time history of vehicle charging during this time. Thruster firings beginning near $176 \mathrm{sec}$ appear to have a major effect on ambient density as well as floating potential. The information provided by the probes in this area could well turn out to influence future design considerations in active space experimentation.

\section{Electron Density}


Figure II.12 and II.13 show upleg and downleg electron density profiles. Although affected at times by thruster firings, data reduction techniques associated with the probe pulsing procedure allow one to unfold electron density based upon normalized characteristics. The ionospheric plasma environment at about $103 \mathrm{~km}$ was characterized by the occurrence of Sporadic-E layers both upleg and downleg. These layers were seen to begin about 30 minutes before launch by the ionosonde operating from Los Alamos during the flight. The upleg Sporadic-E layer shows a density as high as $5 \mathrm{X}$ $10^{4} \mathrm{~cm}^{-3}$ whereas downleg this peak density is measured as $2 \times 10^{5} \mathrm{~cm}^{-3}$. If this is in fact the same layer both upleg and downleg, there appears to be a variability with a density difference near a factor of two.

The occurrence of Sporadic-E layers and the level of densities measured by the Langmuir Probes both upleg and downleg in the flight are factors which complicate the ionosonde measurement. Ionization levels near 1 $2 \times 10^{3} \mathrm{~cm}^{-3}$ are too small to be reliably measured by the ionosonde, whose lowest reliable transmission frequency is $1.1 \mathrm{MHz}\left(\mathrm{N} \mathrm{e}=1.5 \times 10^{4} \mathrm{~cm}^{-3}\right)$ (Paul Argo, private communication). For comparison a nighttime height profile of early morning (0545) ionization at white Sands is shown in Figure II.14. It appears from the ionization profiles that the BEAR payload never reached the ledge at about $195 \mathrm{~km}$ which is evident in the earlier profile. In terms of accelerator operation it is significant that during the majority of gun pulses, ambient electron density remained very near $1-1.5 \times 10^{3} \mathrm{~cm}^{-3}$. From a number of considerations related to vehicle charging which are covered below, it is almost certain that ionization levels were in this range (Compare, for example, the effect of high beam current emission and low beam current emission on the electron saturation current levels of Figures II.21 and II.22. Even at the 0.8 ma emission level there is evidence of charging. Ambient current levels to the vehicle at $\mathrm{N}_{e}=10^{3} \mathrm{~cm}^{-3}$ are about $.75 \mathrm{ma}$ which should indicate charging effects for the low beam current. This is in fact the case as seen in Figure II.21)

\section{Electron Temperature}

From Figure II.14 one can see that ambient temperature levels averaged about $500{ }^{\circ} \mathrm{K}$ in the lower altitudes $(110-120 \mathrm{~km})$ gradually increasing to near $1500-18000^{\circ}$ at the upper altitude range $(180-195 \mathrm{~km})$.

Electron temperatures were obtained by conventional analysis procedures used to unfold Langmuir probe characteristics.

\section{Vehicle Charging}

Detection of vehicle voltage levels was made possible by two separate Langmuir probe systems, one of these (the HIV) was specifically designed to assist in voltage determination. All instruments vere capable of sampling during the 50 usec gun pulse.

Two separate methods of voltage level determination were employed with the two instrument types. For the Langmuil: probes. saturation current level 
changes were measured, where bias levels ranged from $-30 \mathrm{~V}$ to $+30 \mathrm{~V}$. For the High Voltage Device (HIV), which is described in Section III, collected current polarity, as based on applied bias levels, is used as the primary indicator of the magnitude of the voltage.

Figure II.16 shows each of the two systems indicating voltage shift polarity during the gun pulse intervals. Looking at the first of the two highlighted regions beginning at 274.4 seconds, the top panel shows the offsetting potential applied to the HIV in order to force collected current to zero (opposite in sign to actual voltage with respect to the ambient plasma), the middle panel shows gun pulses and the final panel, E-probe saturation current. The three current samples near $6 \times 10^{-7} \mathrm{~A}$ in the bottom panel are samples taken within the pulse. They show increasing electron saturation current levels during the pulse; clear indication that the vehicle is charging more positively than the +4.7 volt bias level inside this interval. The top panel similarly shows vehicle potential stepping toward higher positive levels (the offsetting applied voltage steps toward higher negative levels) with each consecutive gun pulse. Since pulses were not regular, HIV data can only be used to show polarity during successive pulses when they occur; the Langmuir probe saturation level changes of the bottom panel are used to determine magnitude, as will be discussed below.

\section{Charging Theory Considerations}

It can be shown from the definition of collected currant, I = Aven,

that

(II.1) $\mathrm{n}=\frac{5.5 \times 10^{10}}{\mathrm{~V}^{\mathrm{k}}} \mathrm{I}_{\mathrm{B}}$

where,

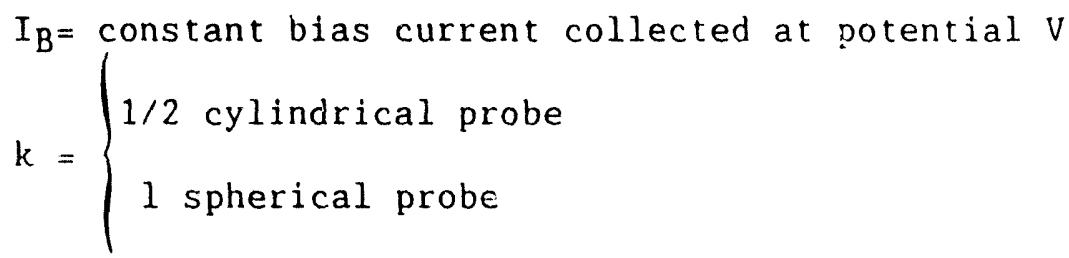

Therefore, if $\mathrm{V}_{0}$ represents our applied bias and we let $k=1$,

(II.2) $\mathrm{n}_{0}=\frac{5.5 \times 10^{10}}{\mathrm{~V}_{0}} \mathrm{I}_{\mathrm{BO}}$

where the subscript 0 refers to current and density levels associated with $V_{0}$. For any $V$, however, (11.1) still applies ot. 
(II.3) $V=\frac{5.5 \times 10^{10}}{n} I_{B}$

Therefore,

(11.4) $V=V\left(n, I_{B}\right), \quad d V=\frac{\partial V}{\partial n}\left|\begin{array}{l}d n+\frac{\partial V}{\partial I_{B}} \\ I_{B}=\text { const }\end{array}\right| \begin{aligned} & d I_{B} \\ & n=\text { const }\end{aligned}$

and.

(II.5) $\left.d V\right|_{n_{0}}=\left.\frac{\partial V}{\partial I_{B}}\right|_{n_{0}} d I_{B}=\frac{5.5 \times 10^{10}}{n_{0}} d I_{B}$

since we assume constant density, $n$, or,

(II.6) $\Delta \mathrm{V}=\frac{\mathrm{V}_{0}}{\bar{I}_{\mathrm{B} O}} \Delta \mathrm{I}_{\mathrm{B}}=\frac{\mathrm{V}_{0}}{\mathrm{I}_{\mathrm{B} O}}\left(\mathrm{I}_{\mathrm{B}}-\mathrm{I}_{\mathrm{B} O}\right)$

This expression may then be used to infer a potential change associated with a saturation current level change from an initial level $\mathrm{I}_{\mathrm{B} O}$.

\section{Observations}

Figure II.17 shows a plot of collected current levels versus pulse periods for the interval 270 to 274 seconds. During this period the beam current emitted was $-.8 \mathrm{~mA}$ and the ambient density was near $10^{3} \mathrm{~cm}^{-3}$. At this density even simple capacitance considerations would imply charging since the ambient plasma can supply about only $.75 \mathrm{~mA}$ to the surface of the accelerator.

Figure II.18 extends the time interval covered from 204 to $313 \mathrm{sec}$ so that all gun pulses sampled during the interval are included as a function of saturation current level (density) before gun firing. What is notable from the figure is that charging levels are as high as 300 volts and that the highest levels occur for the lowest ambient density (i.e., the maximum would occur in vacuum). From a comparison of the IBO levels here to the density profile of Figures II.1.2 and II.13 one concludes that densities over the range covered never exceed about $4 \times 10^{3} \mathrm{~cm}^{-3}$. At these densities one would expect charging to occur at virtually all gun output currents measured. This is in fact consistent with observation.

Moving to Figure II.19 we see a profile of vehicle potential, as derived above, versus time after gun pulse. This Figure tends to show almost 
two separate magnitudes or charging voltage levels with nearly the same rise and decay time i.e., curve l, associated with higher densities in Figure II.18 has a peak after about $10 \mathrm{usec}$ from the onset of the gun pulse emission and decays rather slowly throughout the remaining interval. Similar behavior is seen in curve 2. (The break in the data shown here in the two curves has not been fully analyzed and so at this stage must be considered a speculation.)

\section{Evidence of Ringing}

Figure II.20 shows what is perhaps the most intriguing aspect of the vehicle potential measurement. Although the maximum positive voltage excursion can only be said to be greater than 70 volts because of an electronics saturation problem, the subsequent negative going large excursion occurring after beam shut off would imply, based on the inferred potential, charging to a kilovolt level. These measurements were taken during the "Neutralizer-off" period when output current levels were near $-10 \mathrm{~mA}$ for all pulses. Essentially each pulse with measurable current (at least near - lmA) showed this ringing effect to negative potential after gun turn-off. The characteristic period for return to pre-saturation levels, however, was much larger than a pulse period. These times were more on the order of the ion gyrofrequency as seen in Figure II.21 where the relaxation time is estimated to be about $41 \mathrm{msec}$. ( $\mathrm{f}_{\text {not }} \sim 30 \mathrm{~Hz} \Rightarrow \tau_{\mathrm{fnot}} \sim 30 \mathrm{msec}$ ). This ringing effect has been predicted by a number of theoretical codes (5) and work is proceeding in this area.

Comparisons of the effect of low and high beam current on saturation levels is shown in Figure II.22 and II.23. The most obvious effect which differentiates the two visually is the high level of ion saturation current seen in the high beam current data. This is part of the ringing effect described above.

\section{The Effect of Thruster Firings}

The effects of thruster firing has variable and at times unpredictable effects on instrument function and ambient density levels. These effects are clearly seen in Figures II.24 through II.26. In Figure II.24 electron saturation current is plotted against voltage indicators of pitch/yaw nozzle firing activity. Based purely on this plot one would conclude that the primary effect of the thruster firings was a reduced density in the payload vicinity (recall that electron saturation current is approximately proportional to density through Equation II.1). However, the plot of Figure II. 25 shows that there is more than a simple density effect present. Electron saturation current levels are affected not only by ambient density, but as seen in Equation II.1 by the applied voltage (i.e., in this case the floating potential). The two sweep segments beginning at about 242.6 secs show net electron saturation current collection when the applied sweep voltage is - 3 volts. This implies a vehicle potential shift positively of maybe $4-5$ volts if the potential shifts are associated with the firings (this is not completely clear at this stage of analysis). These two observations (of apparently positive increasing bias with respect to the plasma and, 
simultaneously, decreasing density) are inconsistent since a more positive voltage should imply larger levels of electron saturation current collection. In all probability, the explanation does not lie with the simple relationship expressed in Equation II.1. To further complicate the matter, Figure II.26 shows HIV response to thruster firings at a particularly active thruster firing stage. The response indicates the vehicle charging negatively with respect to the ambient medium. As this instrument is separated from the payload by nearly 2 meters, it is not obvious what implication this has for the region closer to the body.

\section{Over-Neutralization}

As described elsewhere in the report, the BEAR accelerator neutralizer was programmed beginning at about 386 seconds to over-neutralize the $\mathrm{H}$ - beam. This produced a net positive current from the vehicle since $\mathrm{H}$ - ions are doubly stripped. Our indications are that the net current emerging from the vehicle after that time was primarily negative. That this is the case can be seen in Figure III.11 beginning about $380 \mathrm{sec}$. From Figure II.27 this produced a net density increase about the vehicle. This conclusion arises from the observation that both electron and ion saturation currents increase in phase during these pulses. This is a clear indication that higher levels of ionization are somehow being produced. We have not analyzed this data in any detail at the present stage of analysis.

\section{Beam Diagnostic Upleg Ionization Wake}

Figure II.28 shows the combined effect of wake and magnetic field aspect during the ascent of the vehicle. The saturation current levels collected by the probes designed to measure closest to the beam exit environment indeed show the expected effects. There is generally almost an order of magnitude difference discernible in the two traces. As mentioned earlier, once the vehicle assumes an orientation approximately perpendicular to both its velocity vector and the ambient magnetic field, both probes (which are now always in the ram direction and should show neither wake nor magnetic aspect) read identically; they assume initially the same electron saturation current levels as the outer (non-affected) probes show on the upleg portinn immediately before pitchover.

\section{Summary of Vehicle Charging Measurements}

The primary conclusions related to vehicle charging which we can draw from the data at this time are the following:

(i) Derived vehicle charging is as high at 300 volts during accelerator operation, the highest levels occurring at very low densities.

(ii) During the neutralizer-off period vehicle charging (which is a saturated response) is at least +70 volts based only on the saturated level. 
(iii) During low current emissions charging is more apparent at very low density $\left(<1 \mathrm{e} n 3 \mathrm{~cm}^{-3}\right)$. At higher densities, there is still charging but much lower.

(iv) There is ample evidence of ringing after the pulse period on all NRL instrumentation. Inferred voltages based on ion current collection would imply charging to the kilovolt level. This problem is currently under study. 
1. Walker, D.N., and E.P. Szuszczewicz, "Electrostatic Wave Observation During a Space Simulation Beam-Plasma Discharge", J. Geophys. Res., 90, 1691,1985 .

2. Bernstein, W.H., H. Leinback, P. Kellogg, S. Monson, T. Hallinan, D.K. Garriot t, A. Konradi, J. McCoy, P. Daly, B. Baker, and H.R. Anderson, "Electron Beam Experiments: The Beam Plasma Discharge at Low Pressures and Magnetic Field Strengths", Geophys. Res. Lett., 5, 127, 1978.

3. Holmes, J.C., and E.P. Szuszczewicz, "A Versatile Plasma Probe", Rev. Sci. Instrum., 46, 592, 1975.

4. Laframboise, J.G., "Theory of Spherical and Cylindrical Langmuir Probes in a Collisionless, Maxwellian Plasma at Rest", Rep. 100 Inst. of Aerospace Studies, Univ. of Toronto, Toronto, Ont., 1966.

5. Winglee, R.M., "Spacecraft Charging during Electron Beam Injection and Turn-off", Talk presented to Spacecraft Charging Conference, Naval Postgraduate School, R.C. Olsen (Chairman),1-3 November, 1989 (Also submitted JGR Nov 1989) 


\section{BEAM DIAGNOSTICS} (BD) SECTION BEAR PAYLOAD

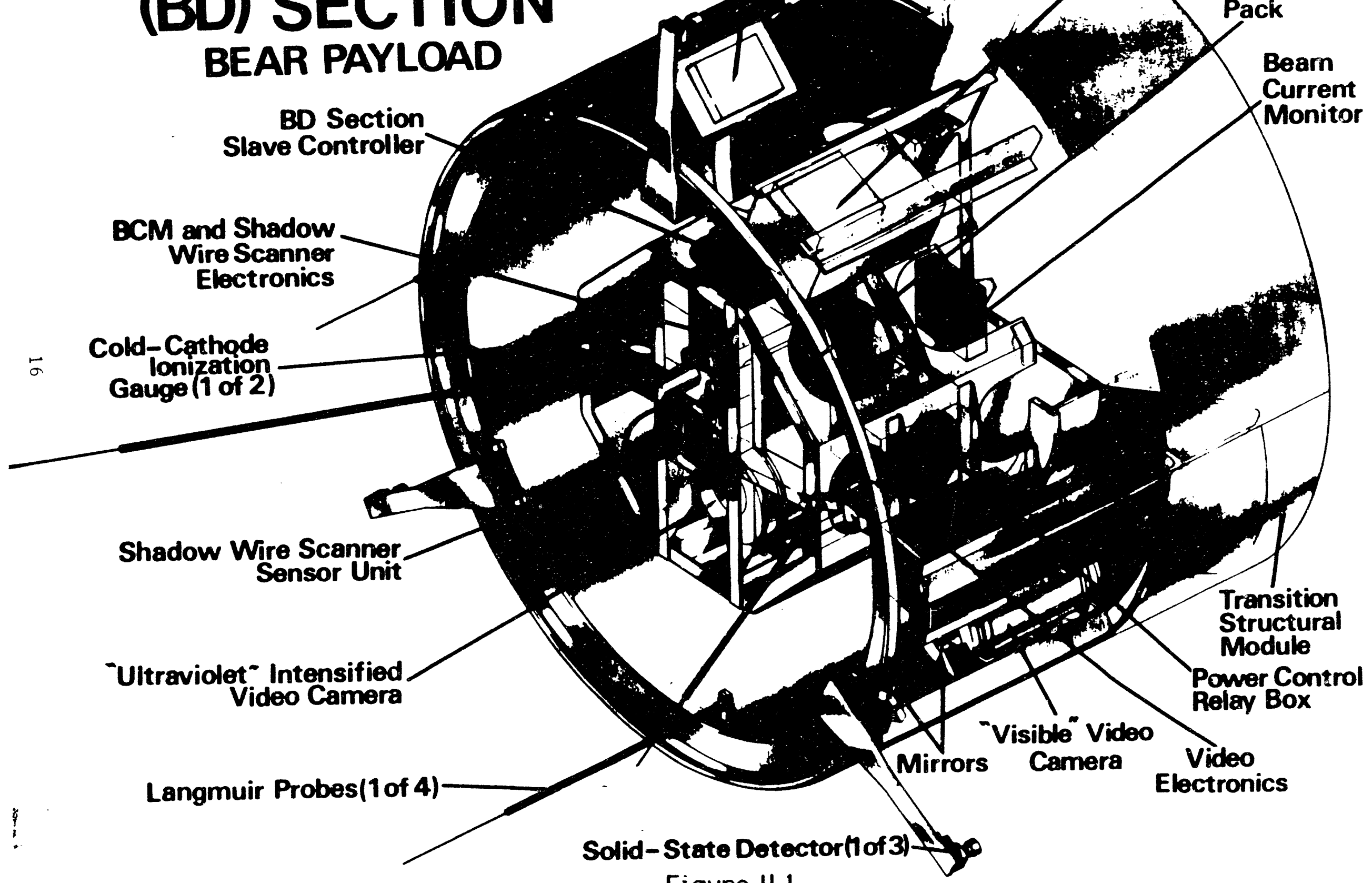

Figure II.1 


\section{OPERATIONAL MODES}

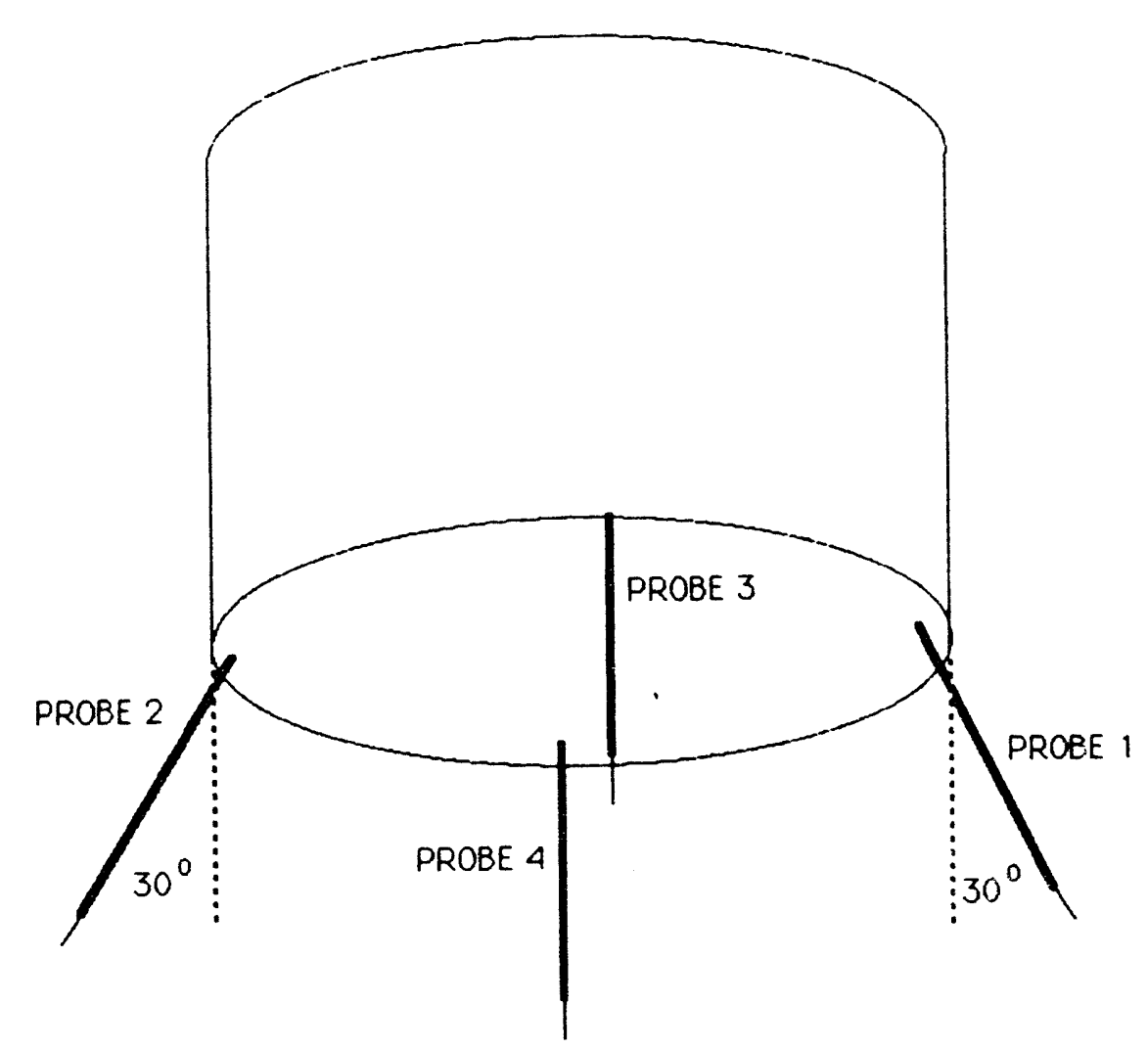

\begin{tabular}{cc} 
SEQUENCING & TIME INTERVAL \\
\hline $1 \& 2$ & 3.2 SEC (16 GUN PULSES) \\
$3 \& 2$ & 3.2 SEC ( 16 GUN PULSES) \\
$1 \& 4$ & 3.2 SEC (16 GUN PULSES) \\
$3 \& 4$ & 3.2 SEC (16 GUN PULSES)
\end{tabular}

Figure 11.2 
VOLTAGE APPLIED TO PROBES

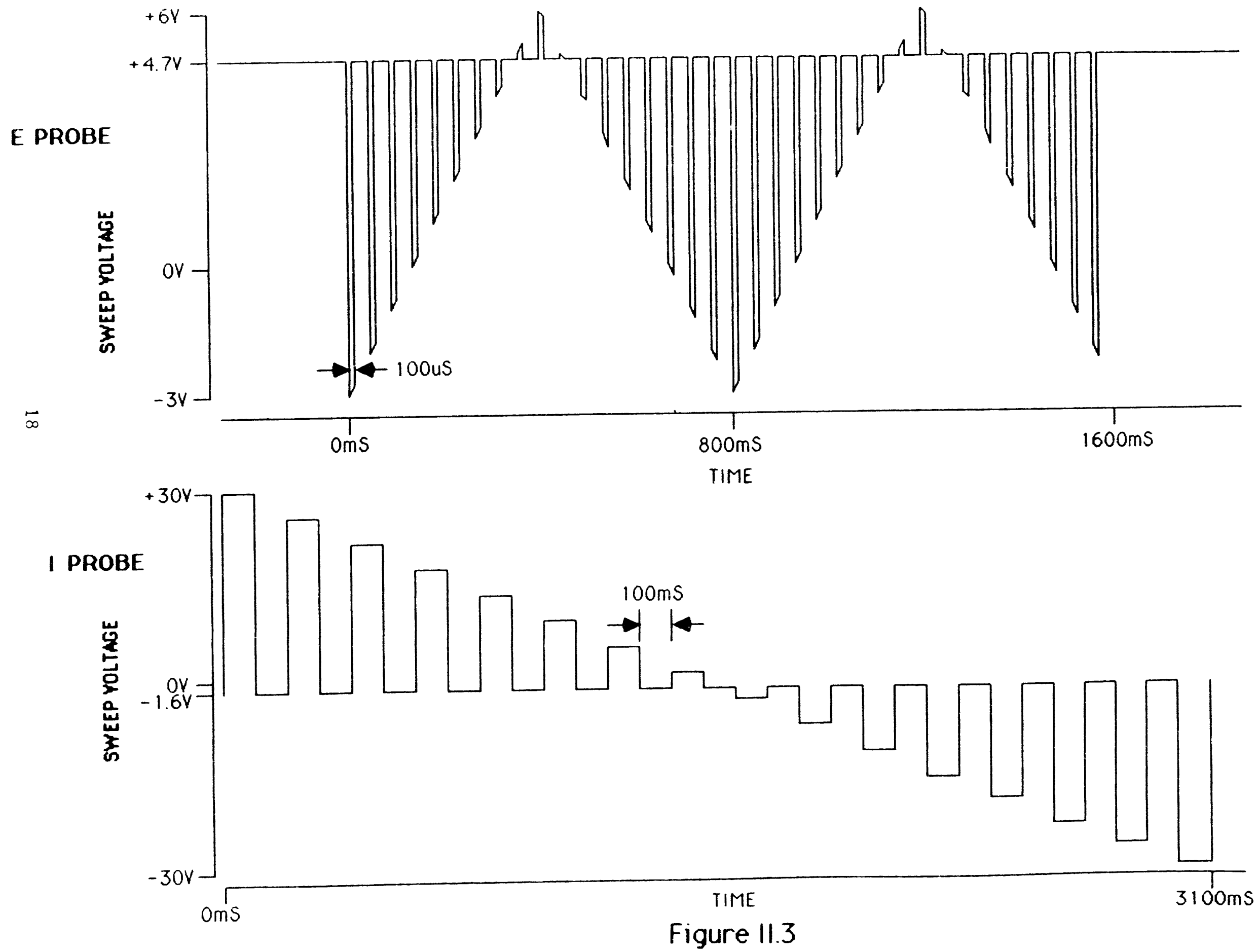


Ŷ. SWEEP GENERATOR SAWTOOTH

SCHMITT CIRCUIT OUTPUT

BEAR OUTBOARD SWITCHING BOX TIMING AND LOGIC

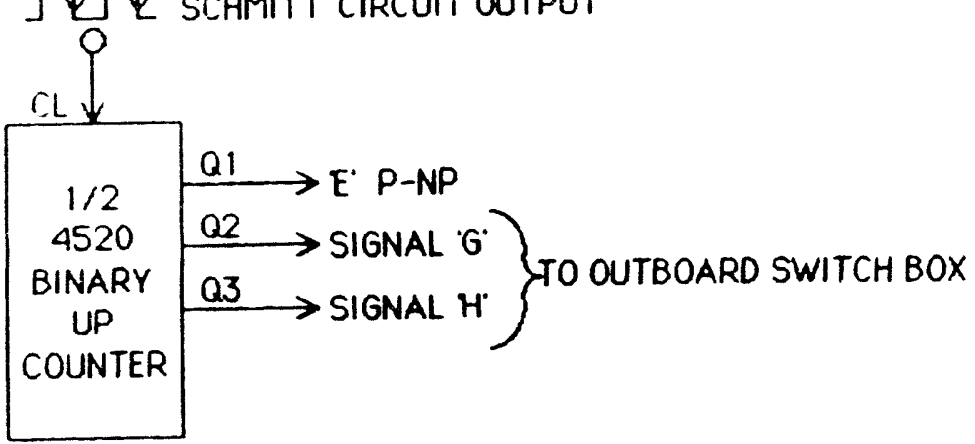

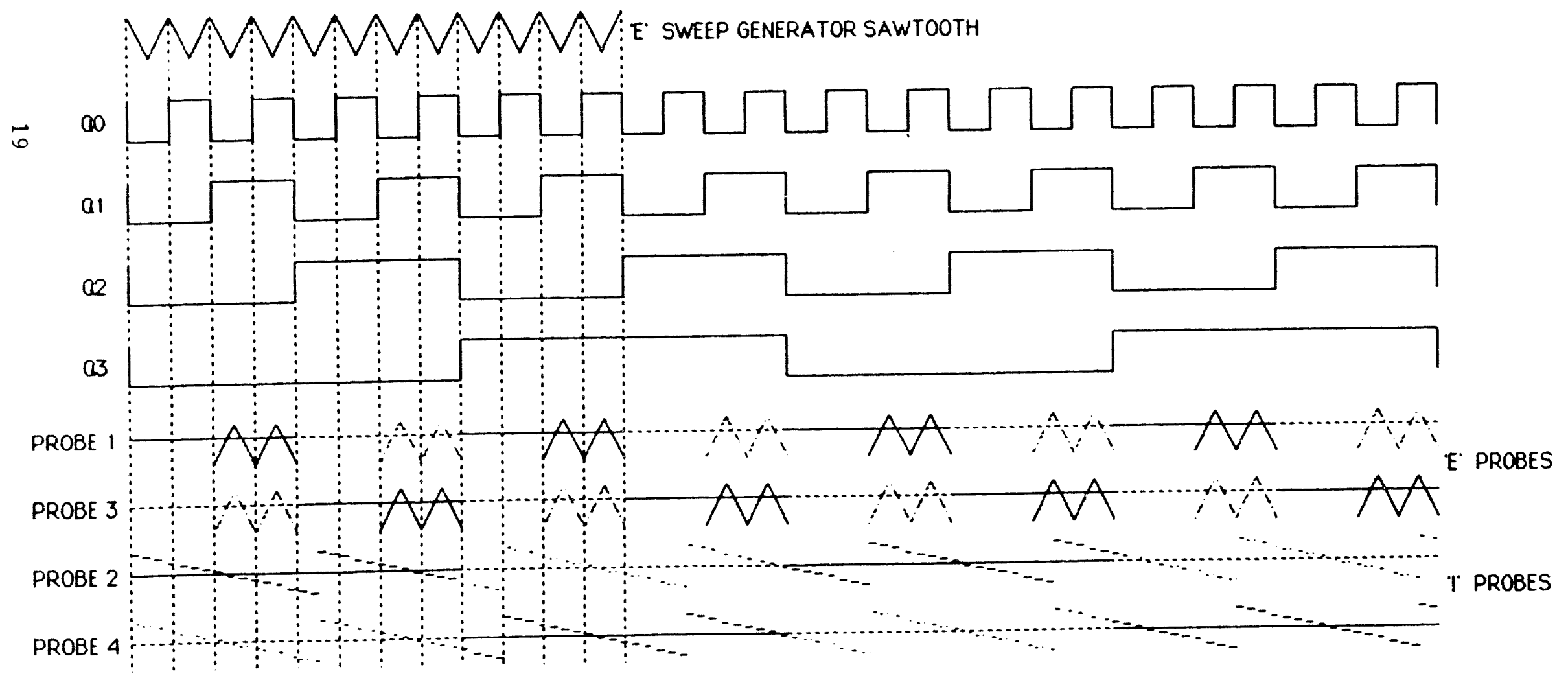

DOTTED LINES MEAN THAT PROBE IS CONNECTED TO SWEEP BUT NOT MONITORED

Figure 11.4 


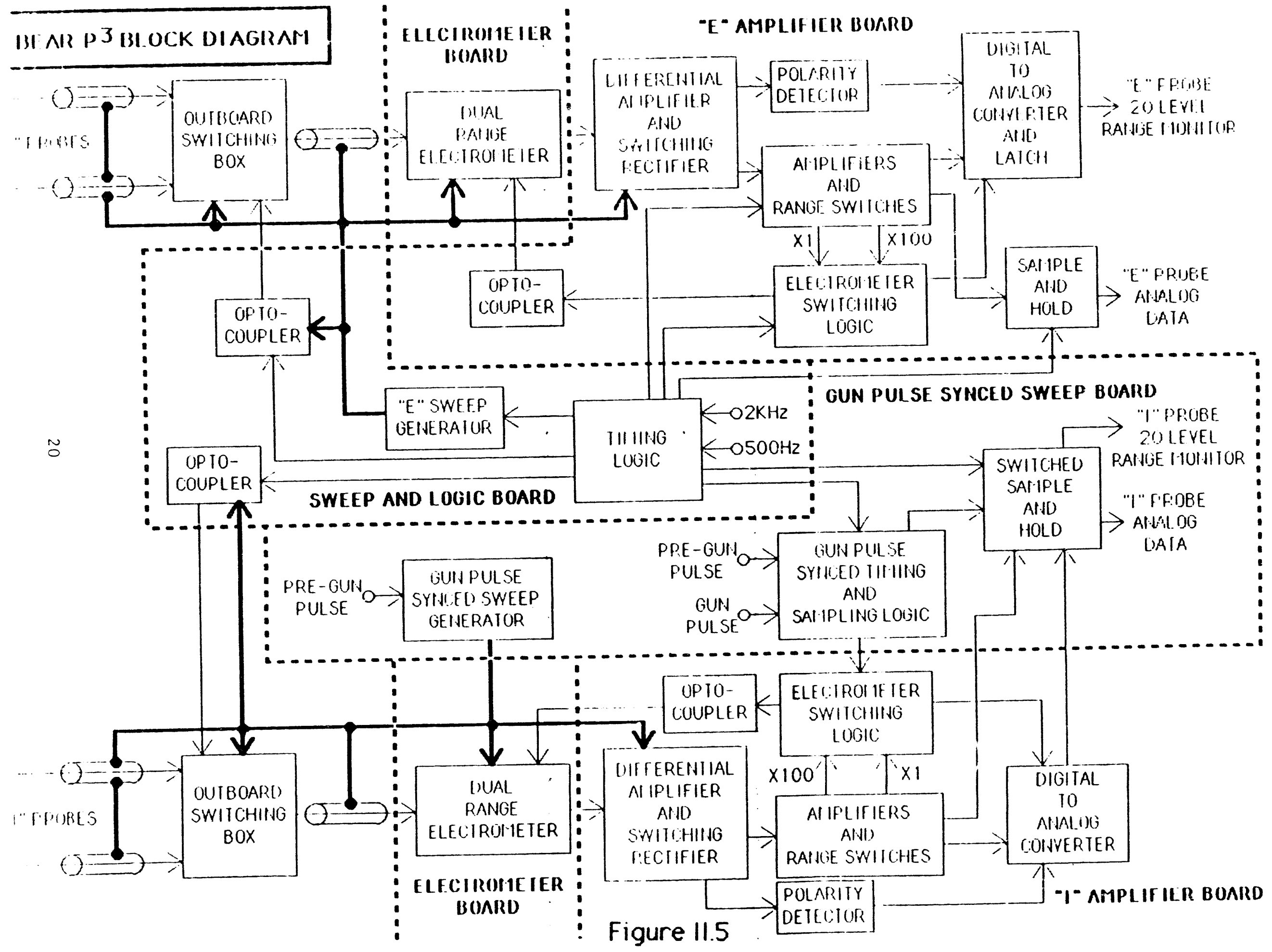




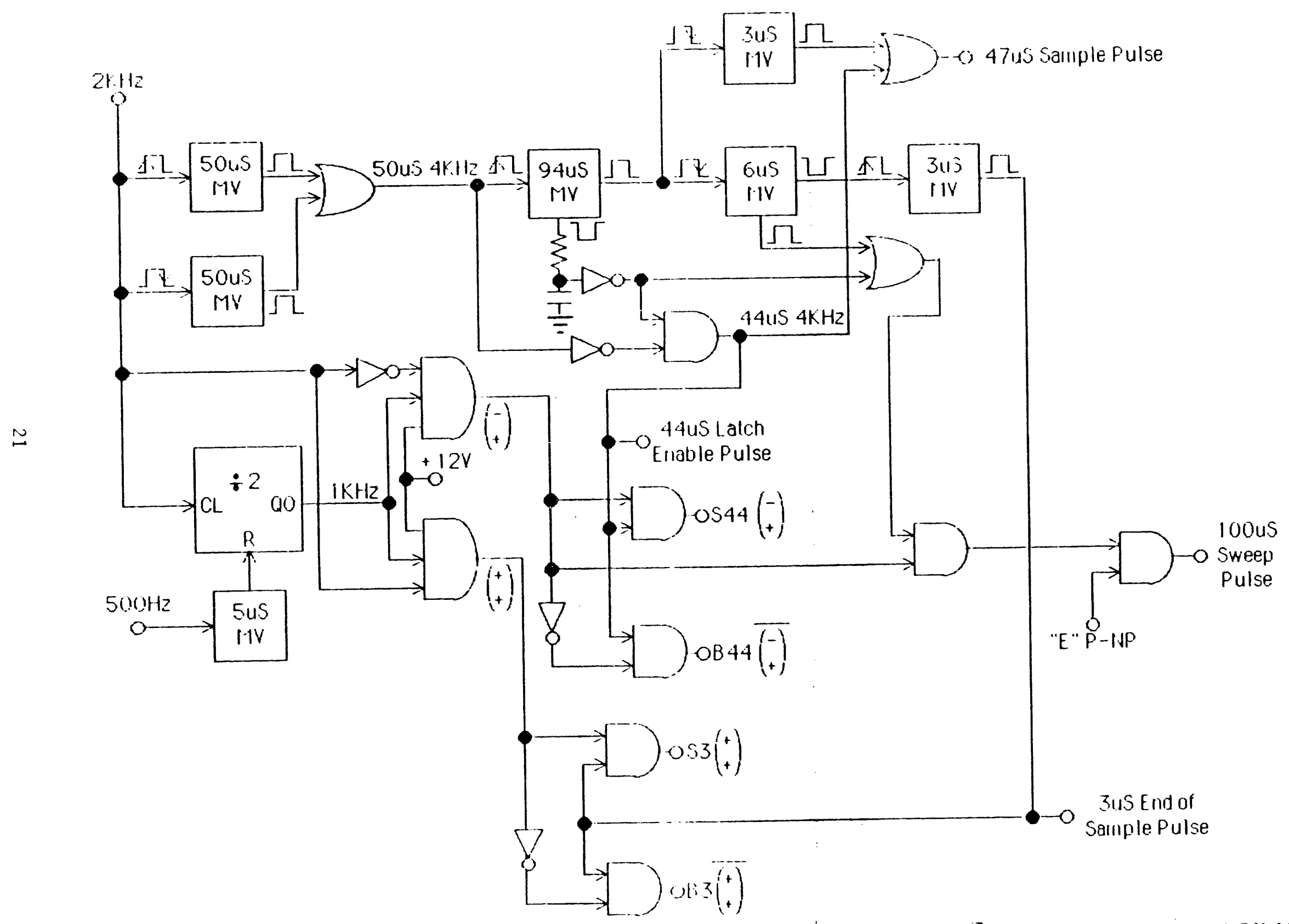

BLAR P 3 I IMING LOGIC DIAGRAM

Figure 11.6 


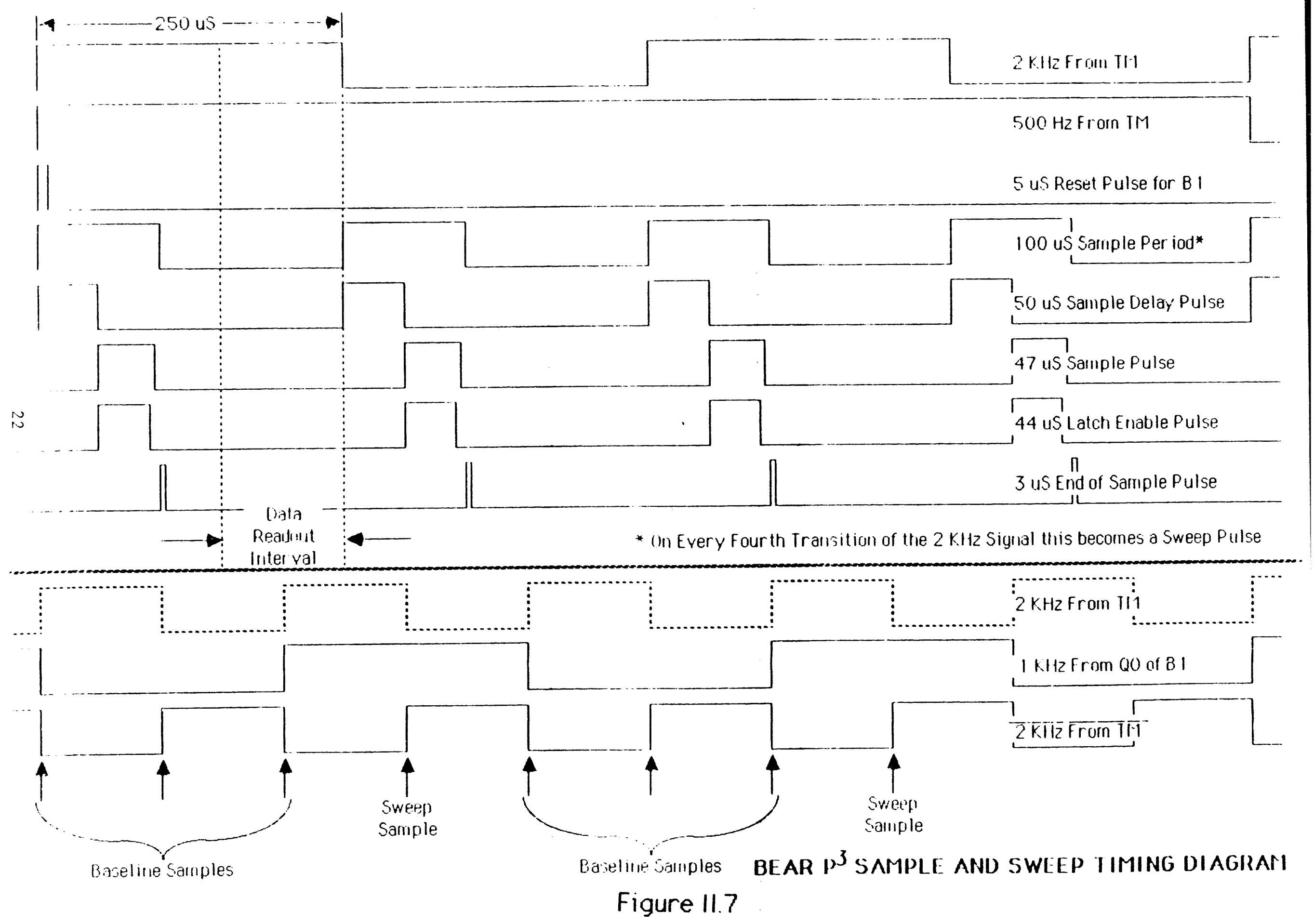




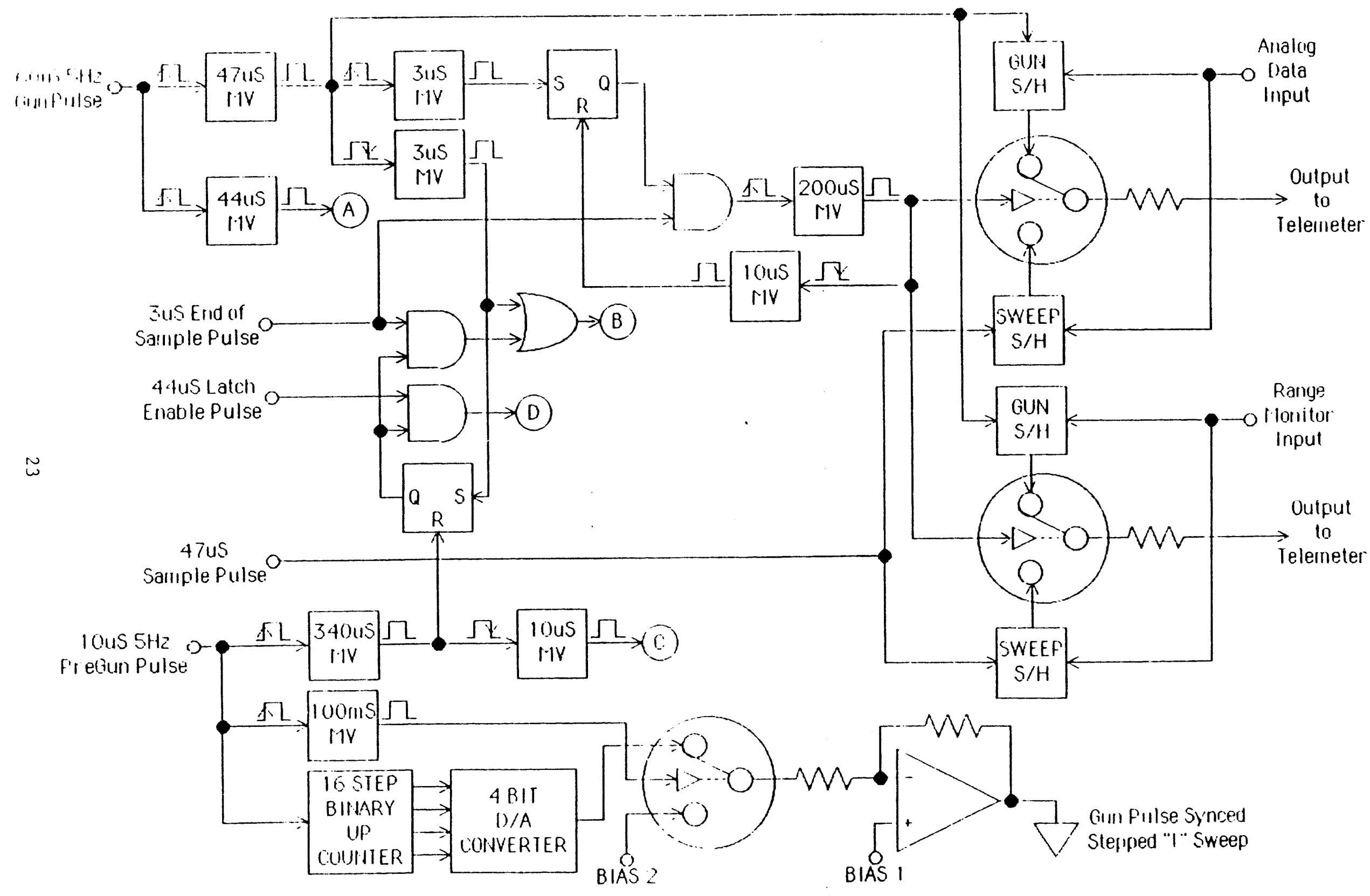

BEAR P3 GUH PULSE SYNCED SWLIP AND IIMING LOGIC DIAGIRAM

Figure 11.8 

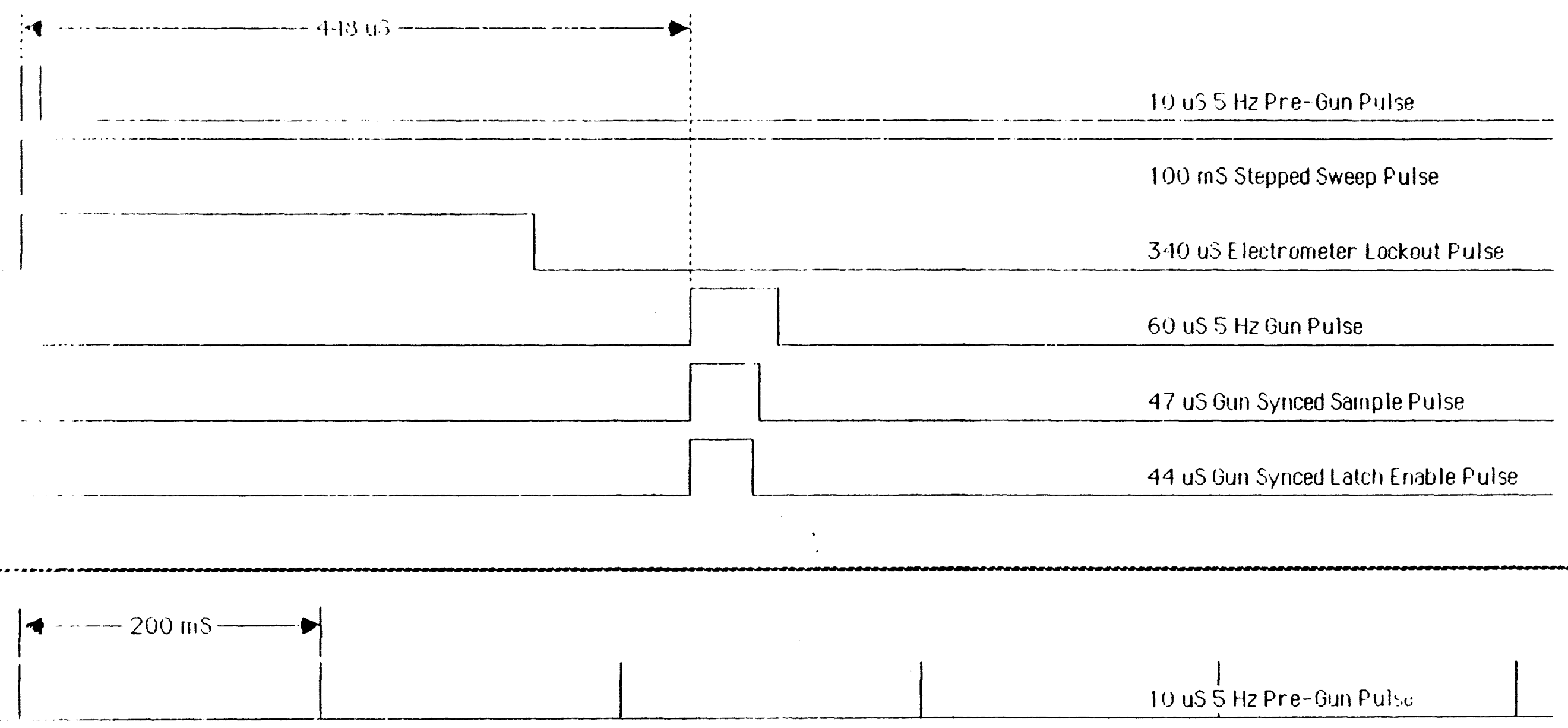

BEAR P ${ }^{3}$ GUN SYNCED SAMPLE AND SWLEP IIMING DIAGRAM

Figure 11.9 


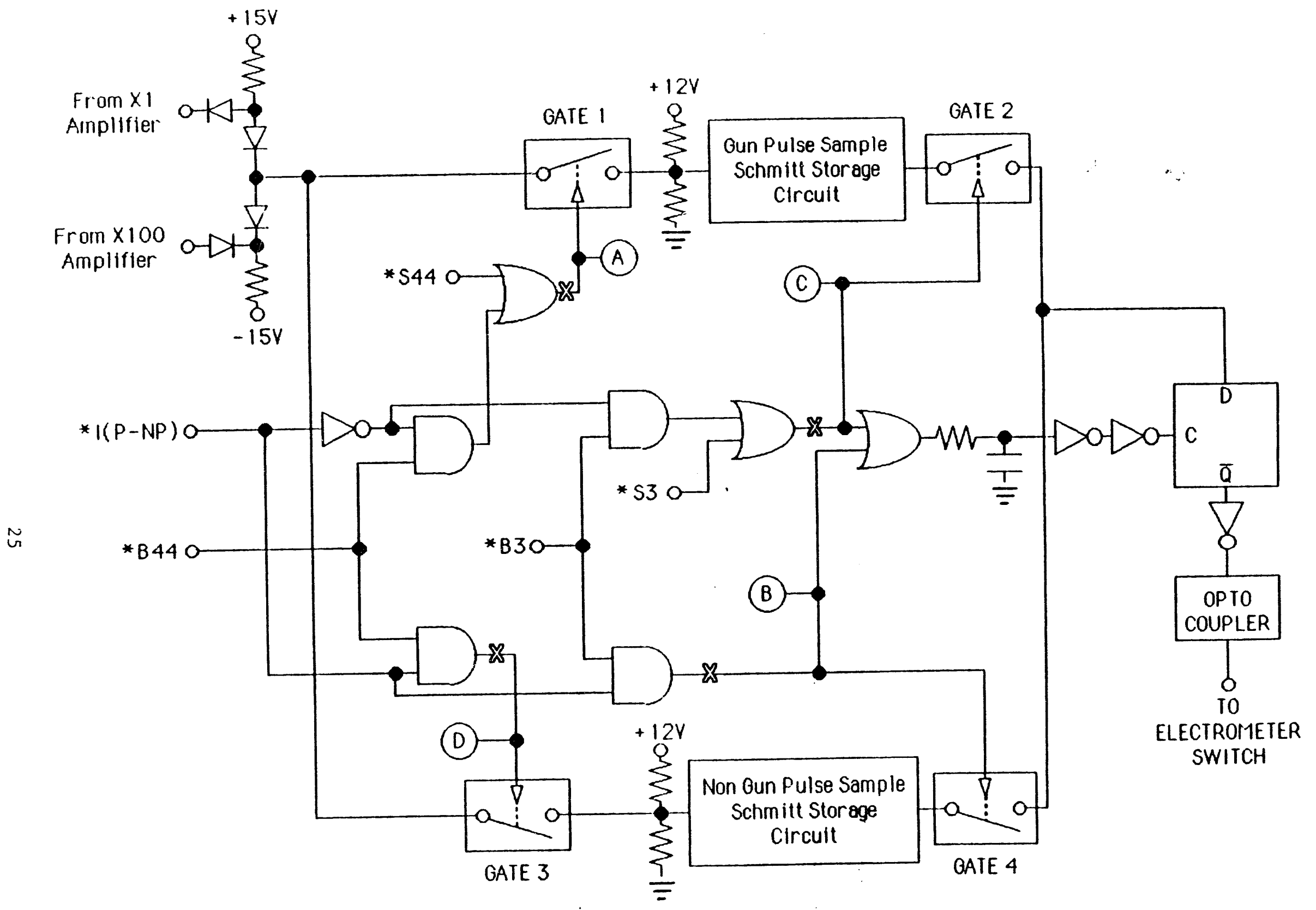

* NO SIGNAL PRESENT

$\because$ ELECTROMETER SWITCHING LOGIC DIAGRAM

Figure 11.10 


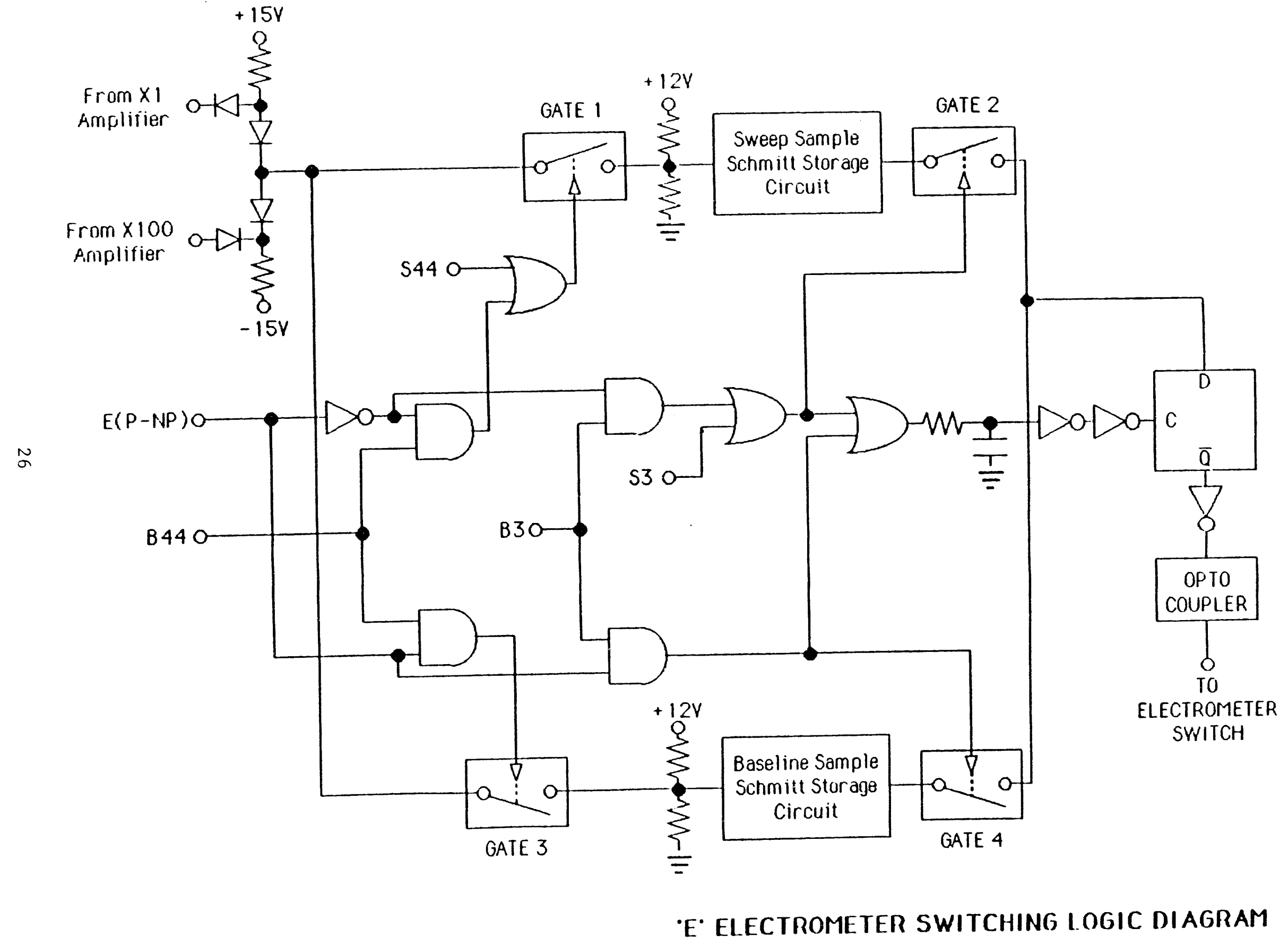

Figure 11.11 


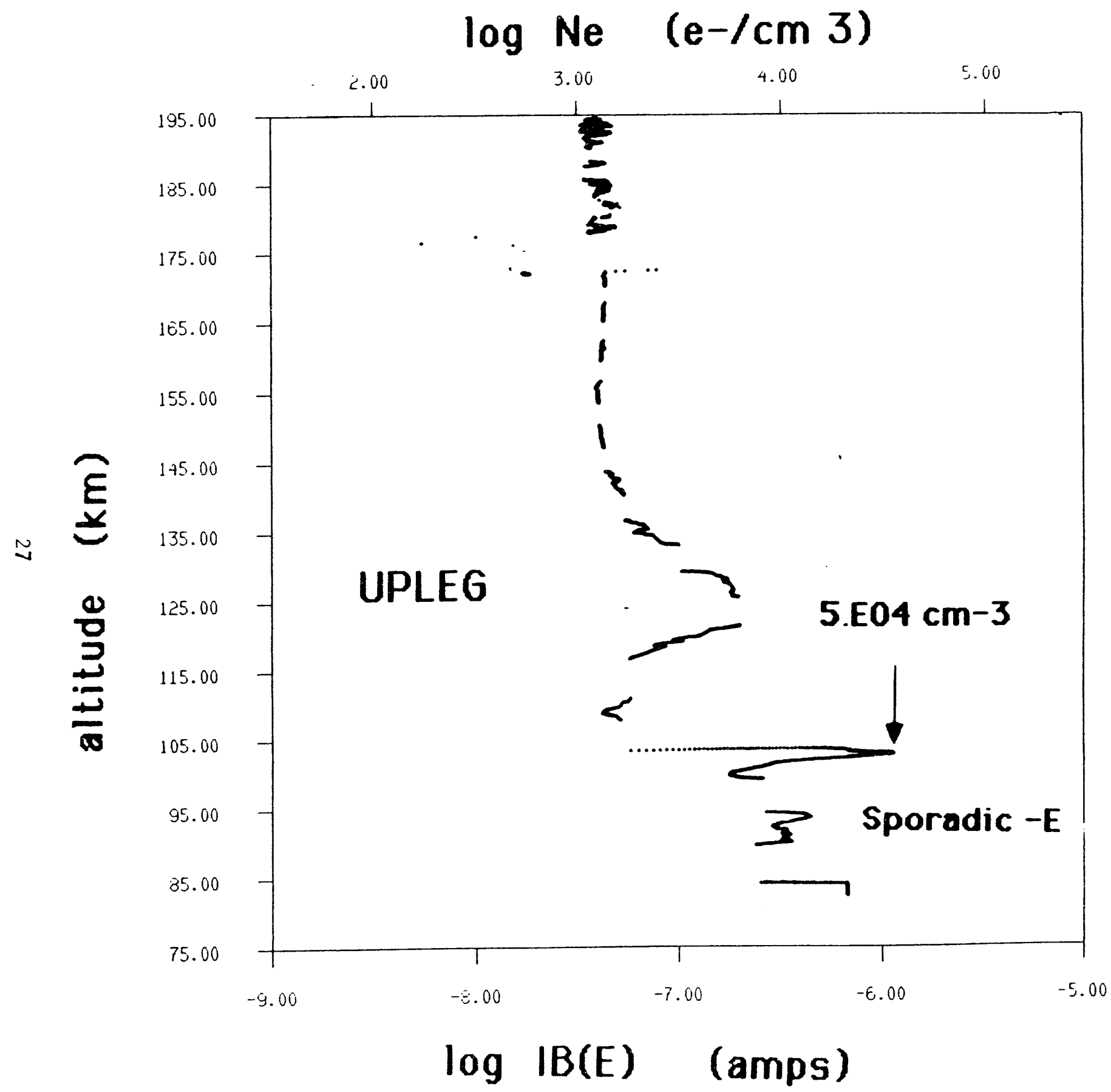

DECIMATION 1:10

SLIDE TO 0.4 SEC

Figure 11.12 


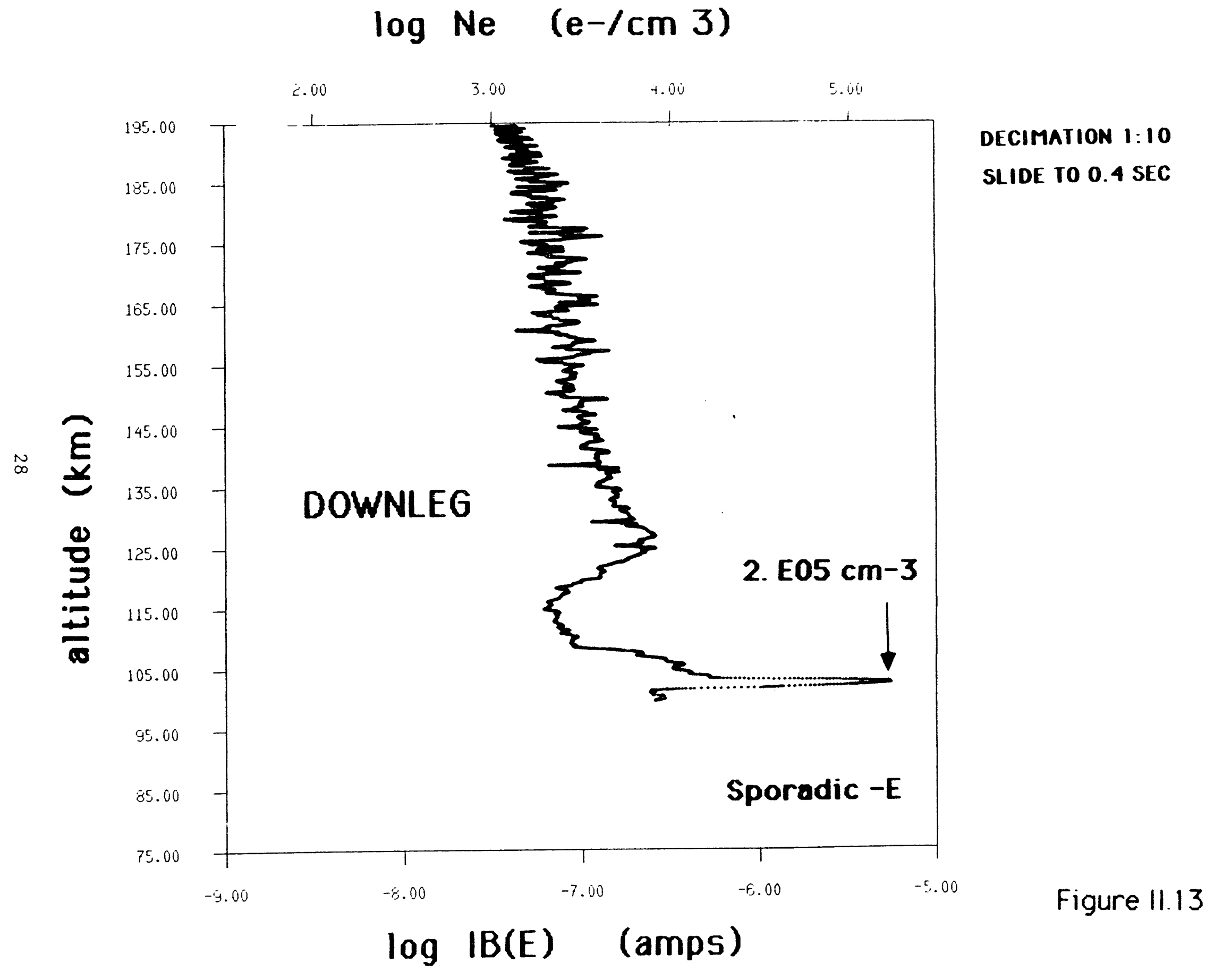




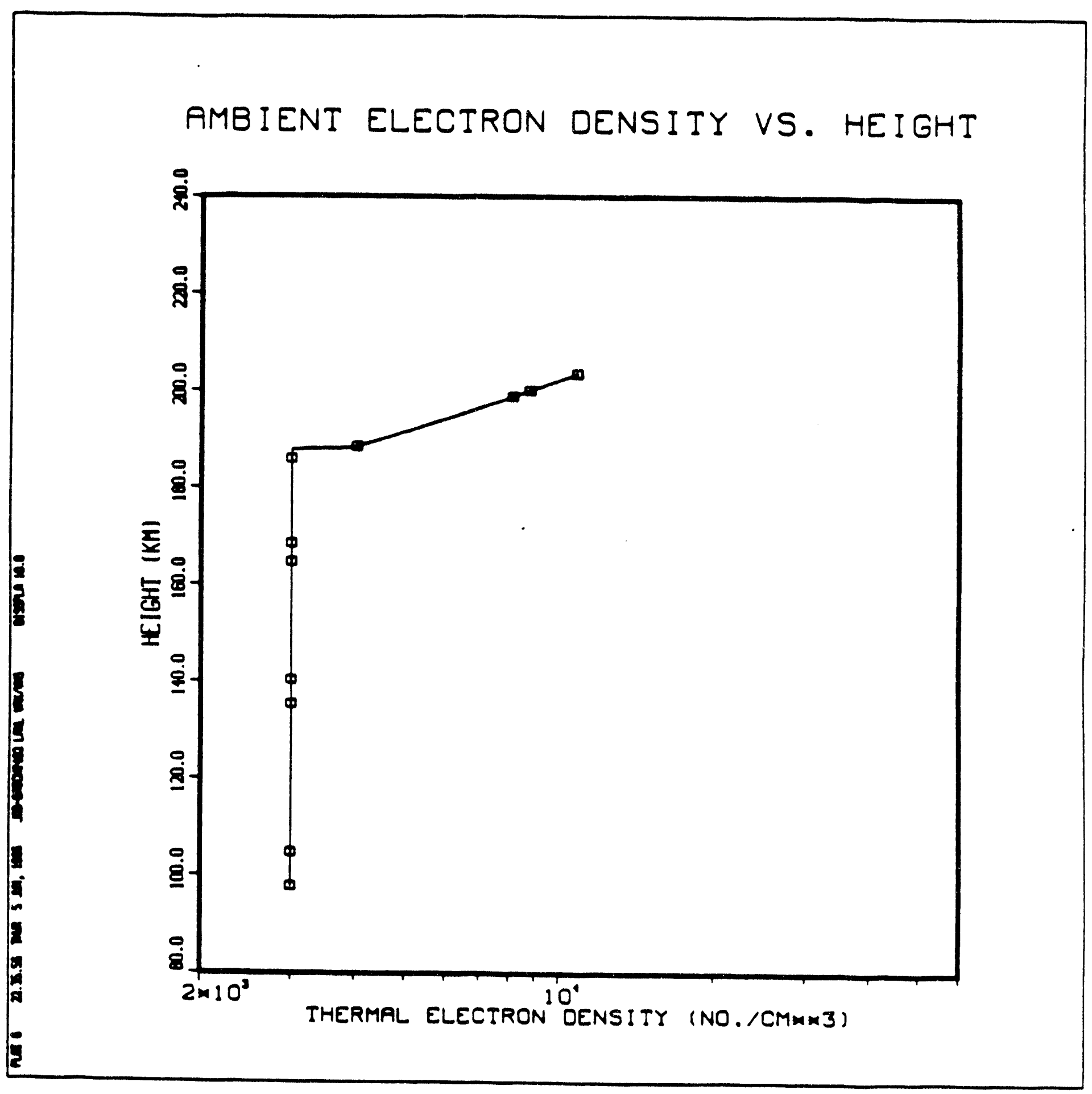

Fig 3.12-1. Nighttime (0545 UT, June 14. 1985) thermel electron density versus height at WSMR (from Rickel, private communication. 1985)

Figure 11.14 
Te vs Altitude....BEAR

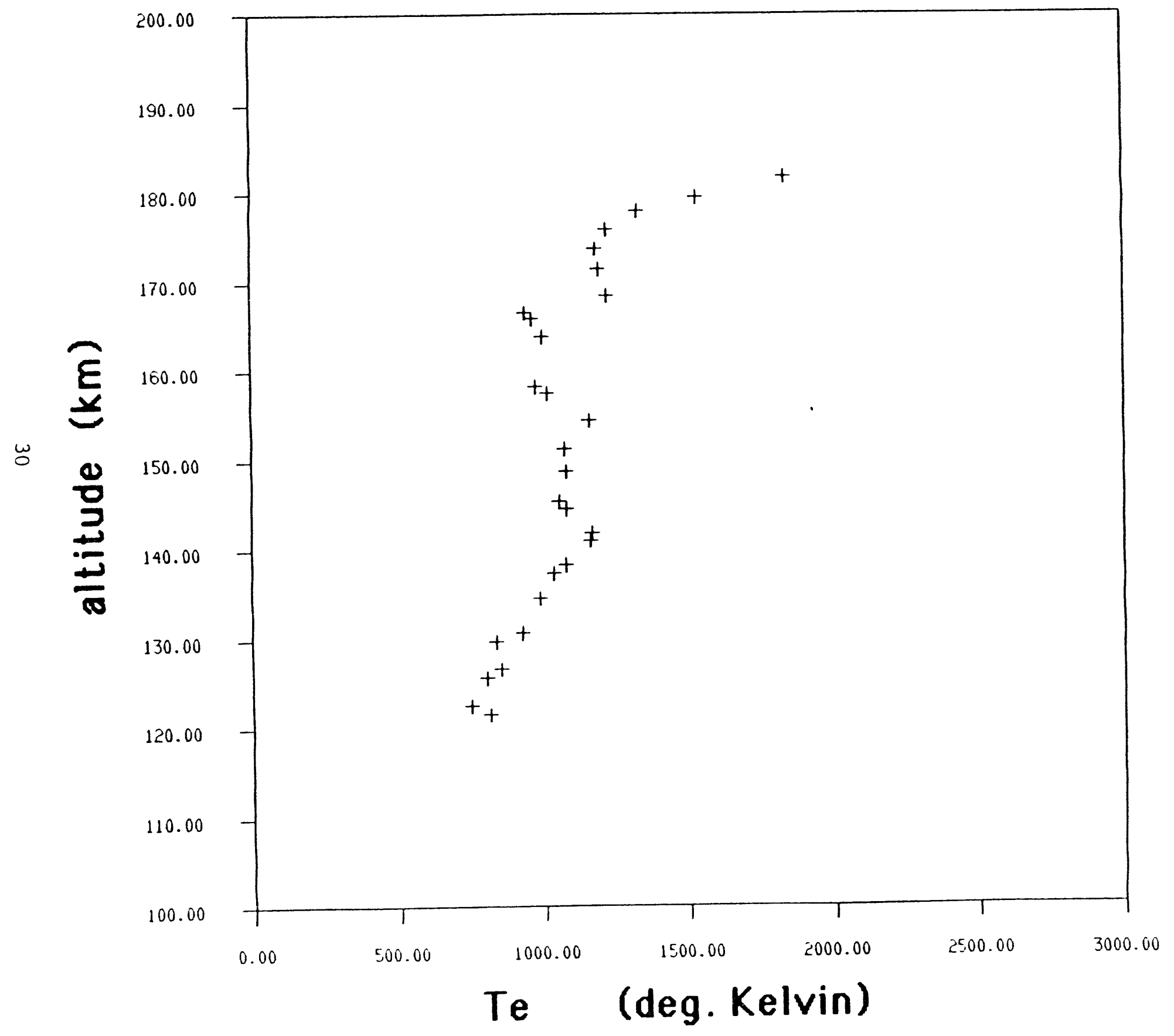

Figure II.15 


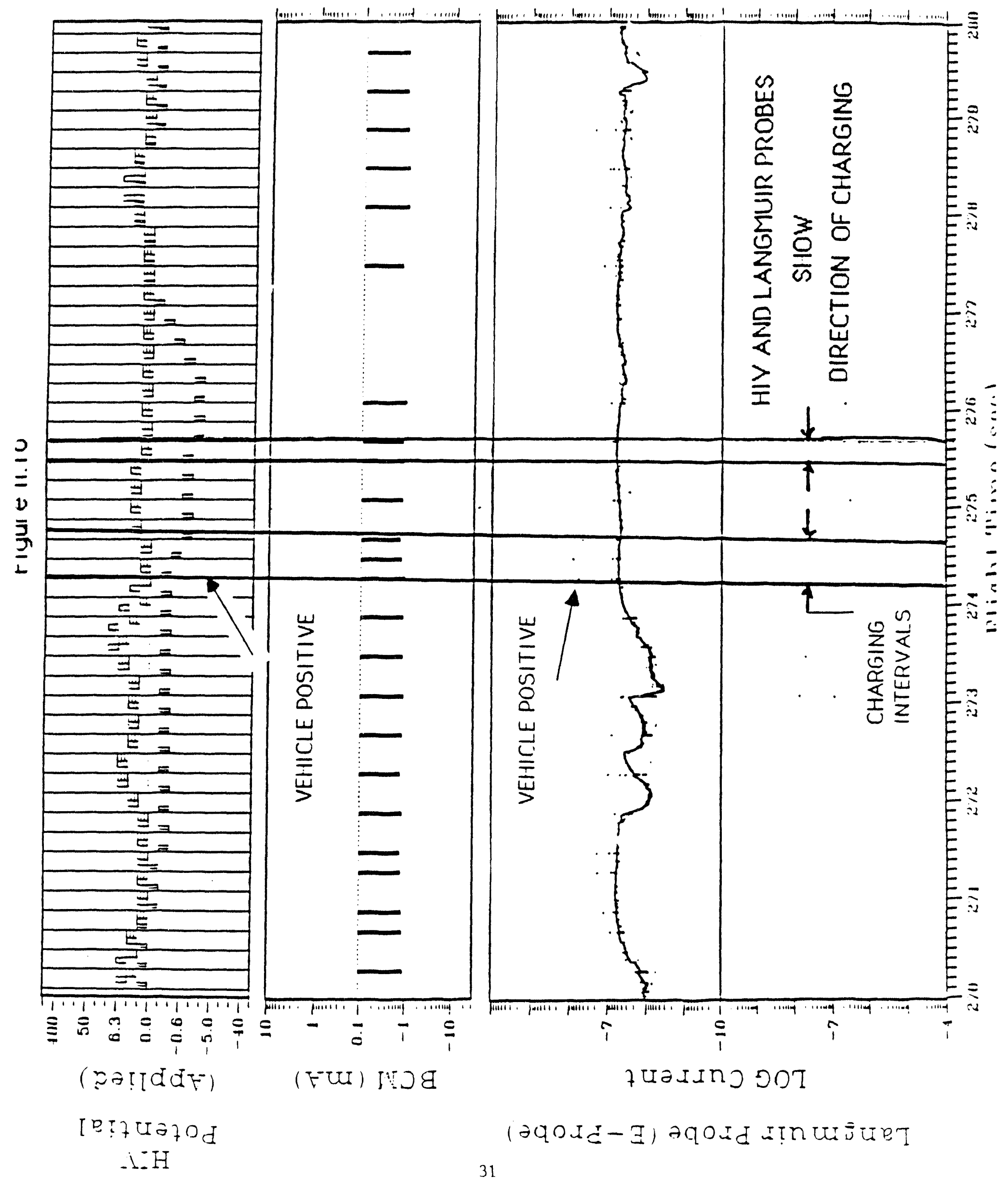


Figure 11.17

\section{NRL}

BEAR...VEHICLE CHARGING...I(BEAM) $=-.8 \mathrm{ma} \mathrm{Ne} \sim 1 . e 03 \mathrm{c}_{1} n-3$

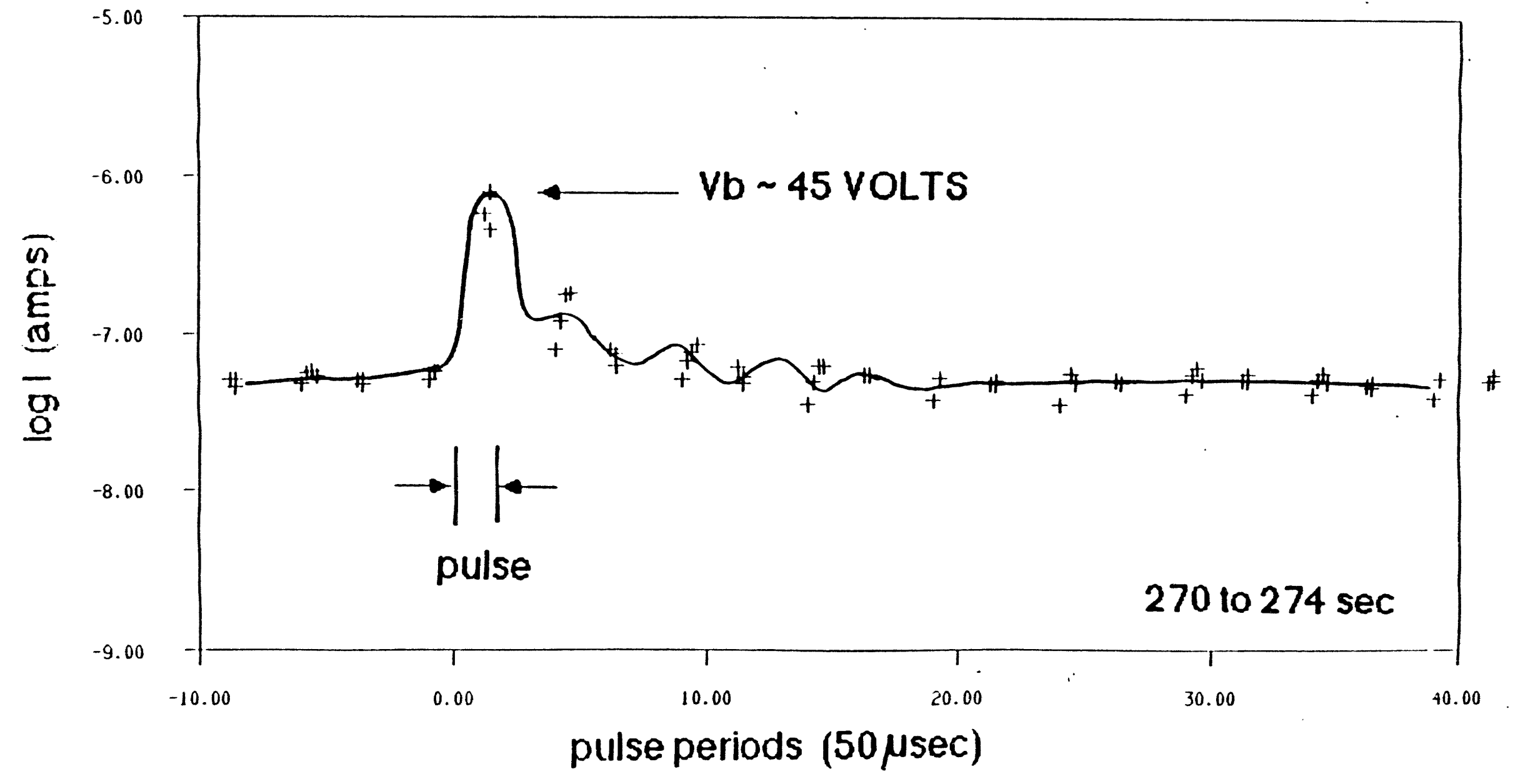




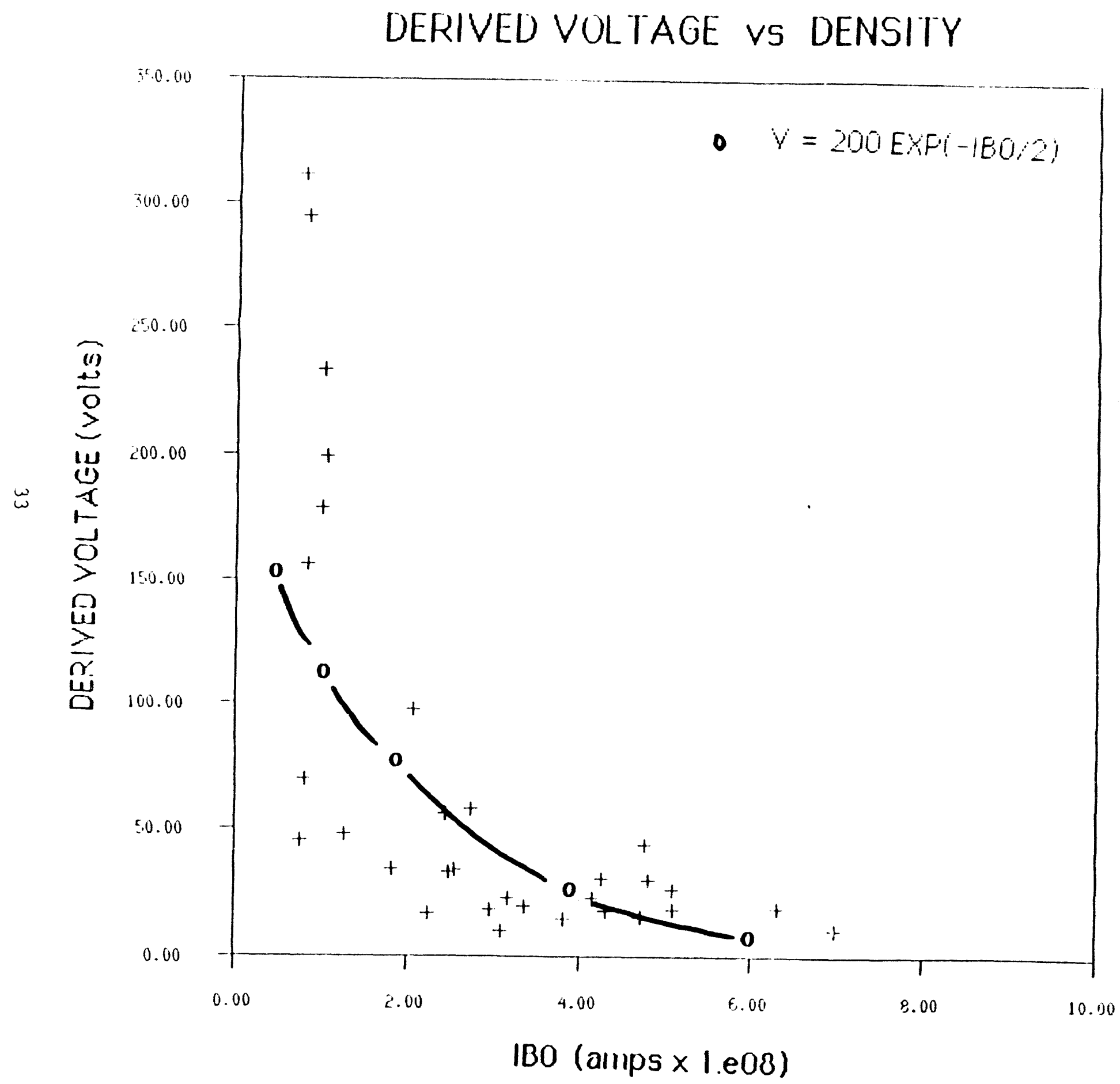

Figure 11.18 


\section{DERIVED VOLTAGE VS TIME}

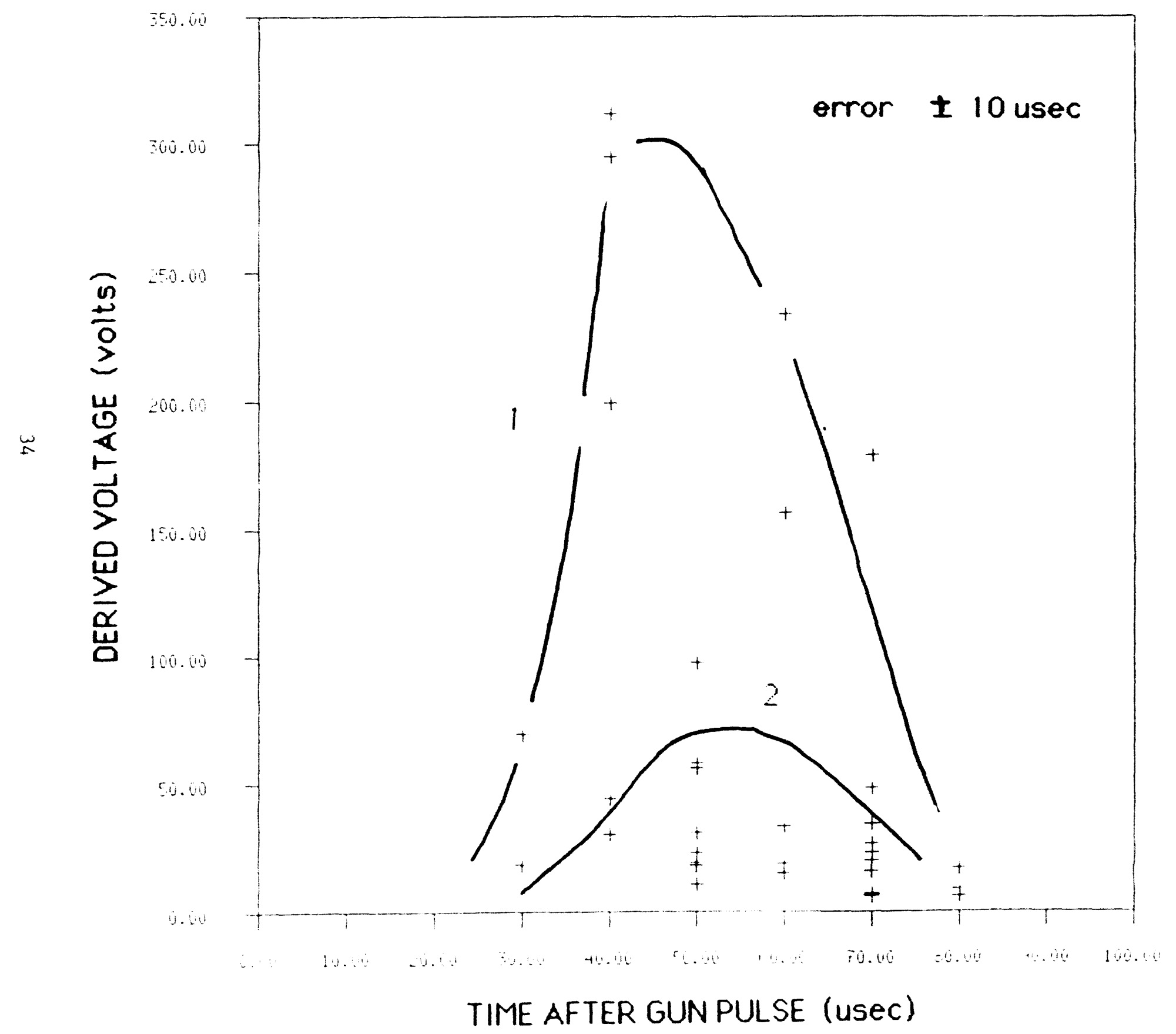

Figure 11.19 


\section{NRL}

BEAR...VEHICLE CHARGING...I(BEAM)=-10 ma $\mathrm{Ne} \sim 1 . e 03 \mathrm{~cm}-3$

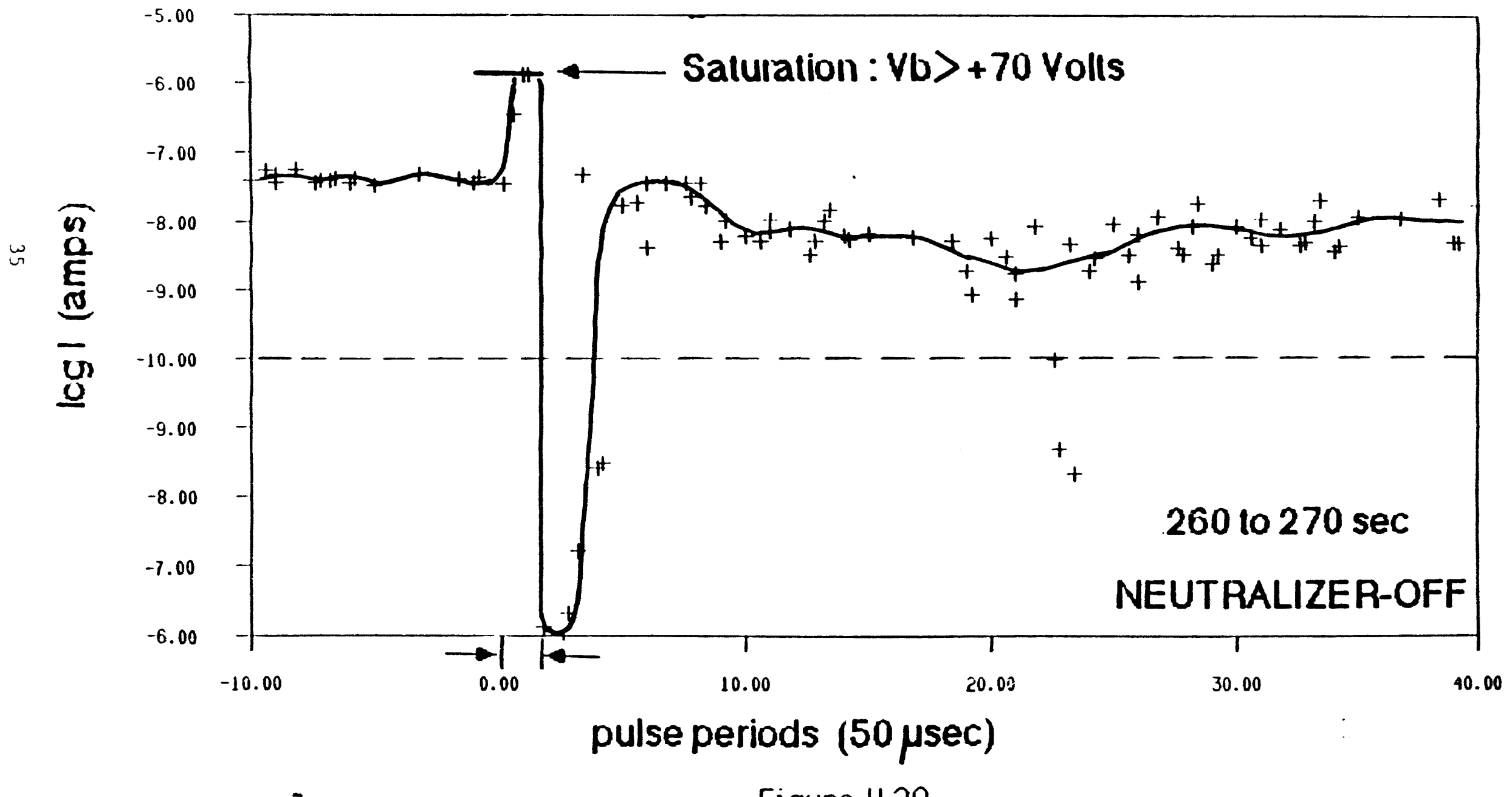




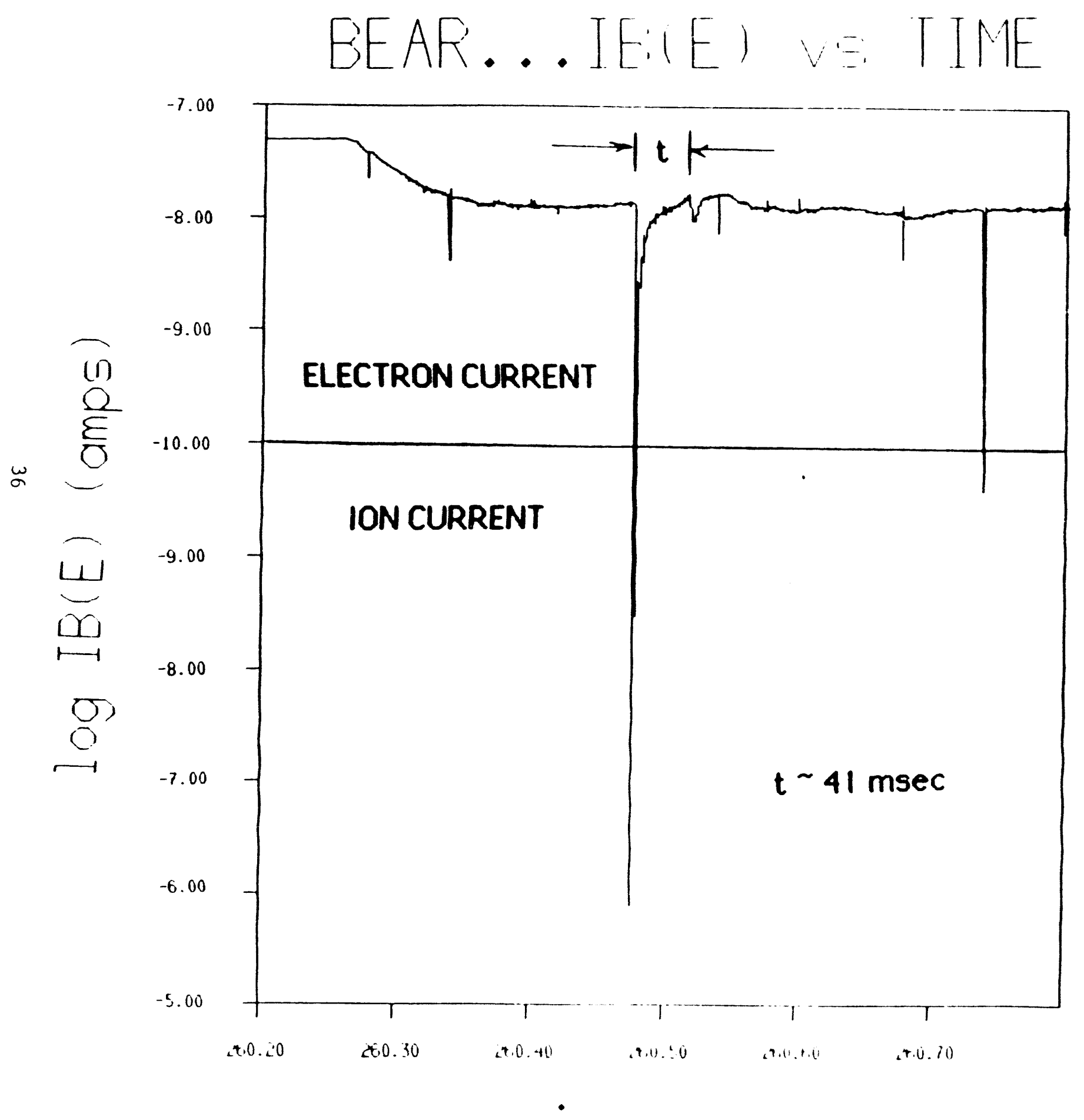




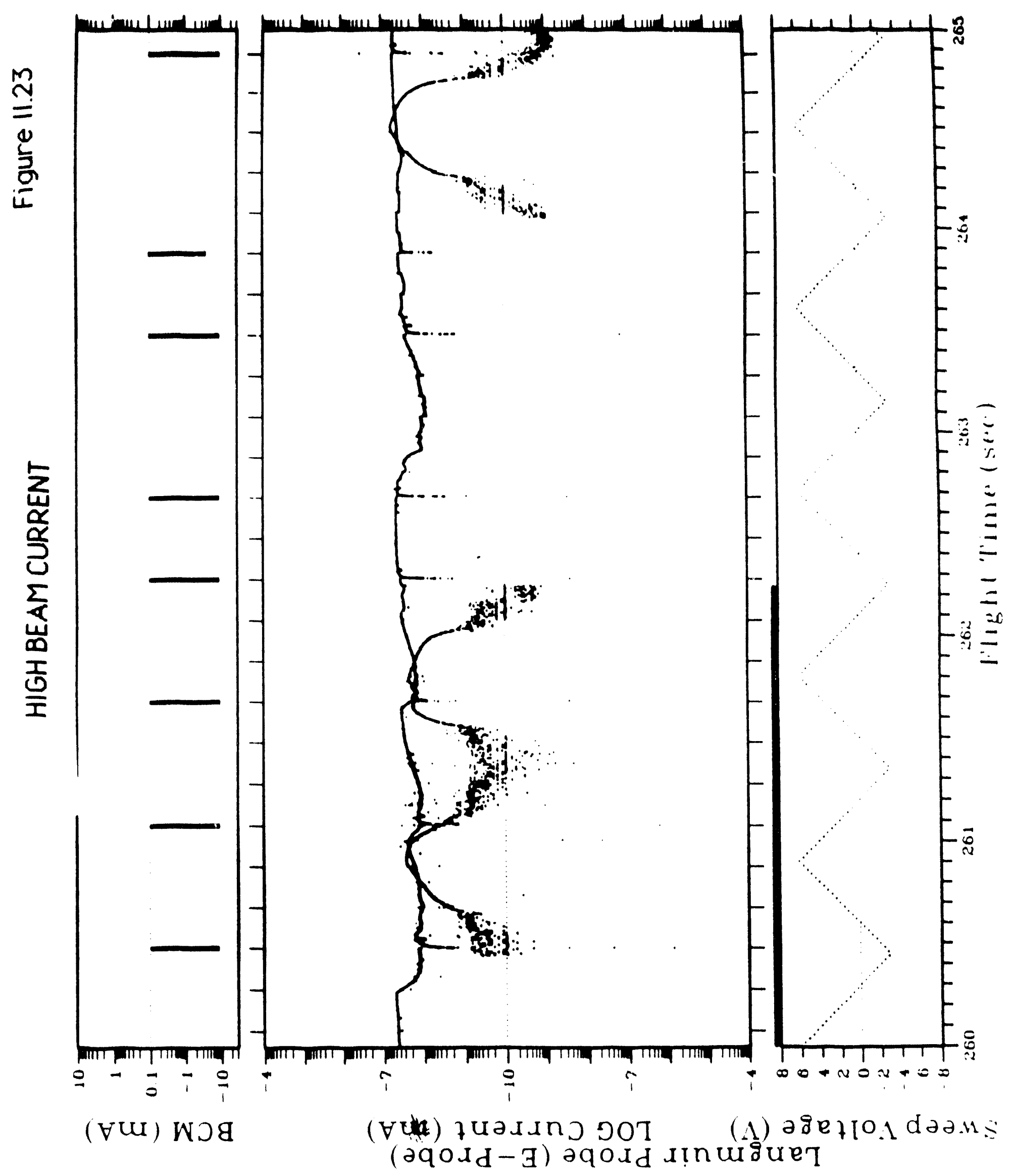


LOW BEAM CURRENT

Figure 11.22
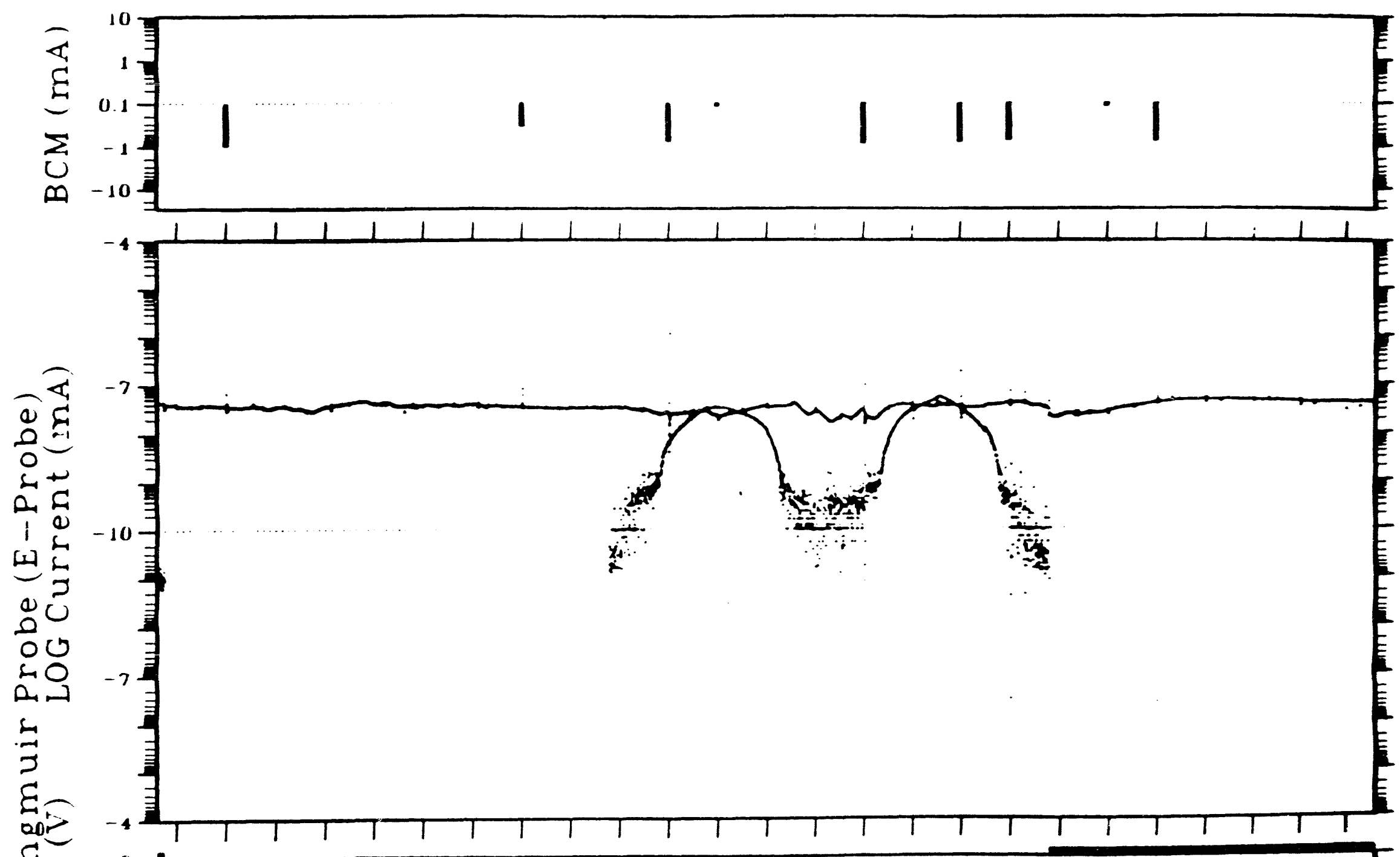

$\begin{array}{cc}0 & 0 \\ 0 & 0 \\ 0 & 1 \\ \omega & 2 \\ \overrightarrow{0} & 0 \\ \overrightarrow{7} & -2 \\ 0 & -1 \\ 0 & -6 \\ 0 & -8-1 \\ \overrightarrow{2} & \end{array}$

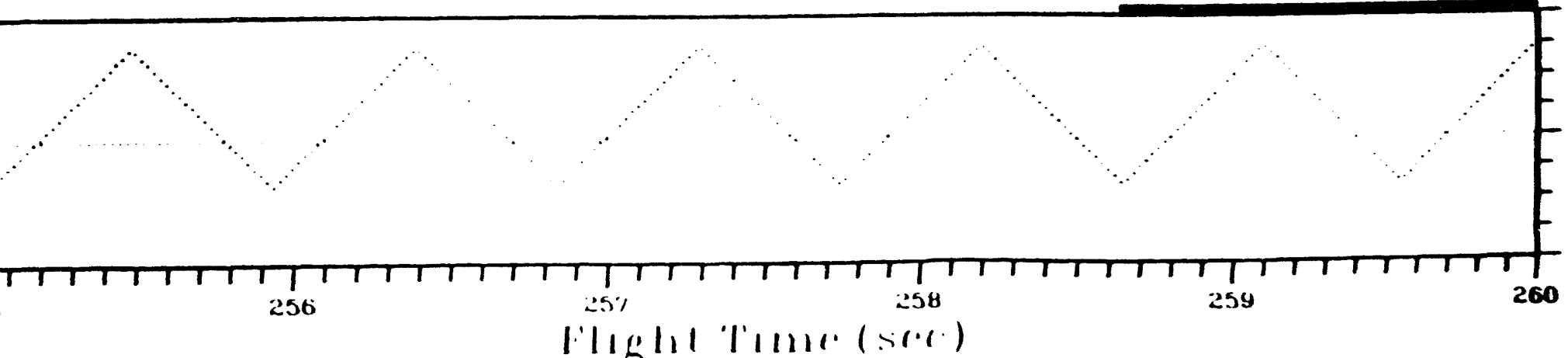


Figure II.24

BEAR...IE (E) VS TIME

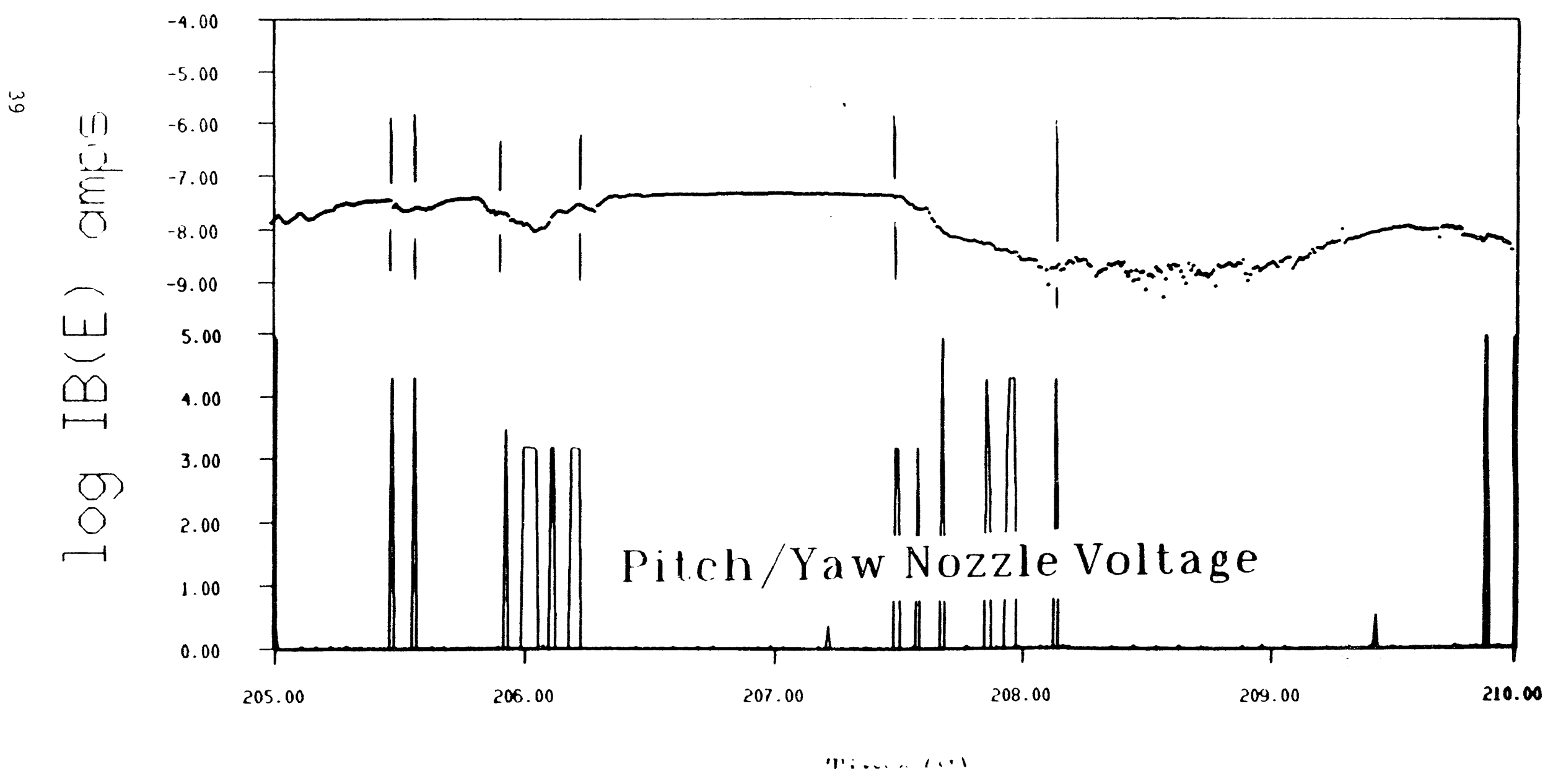




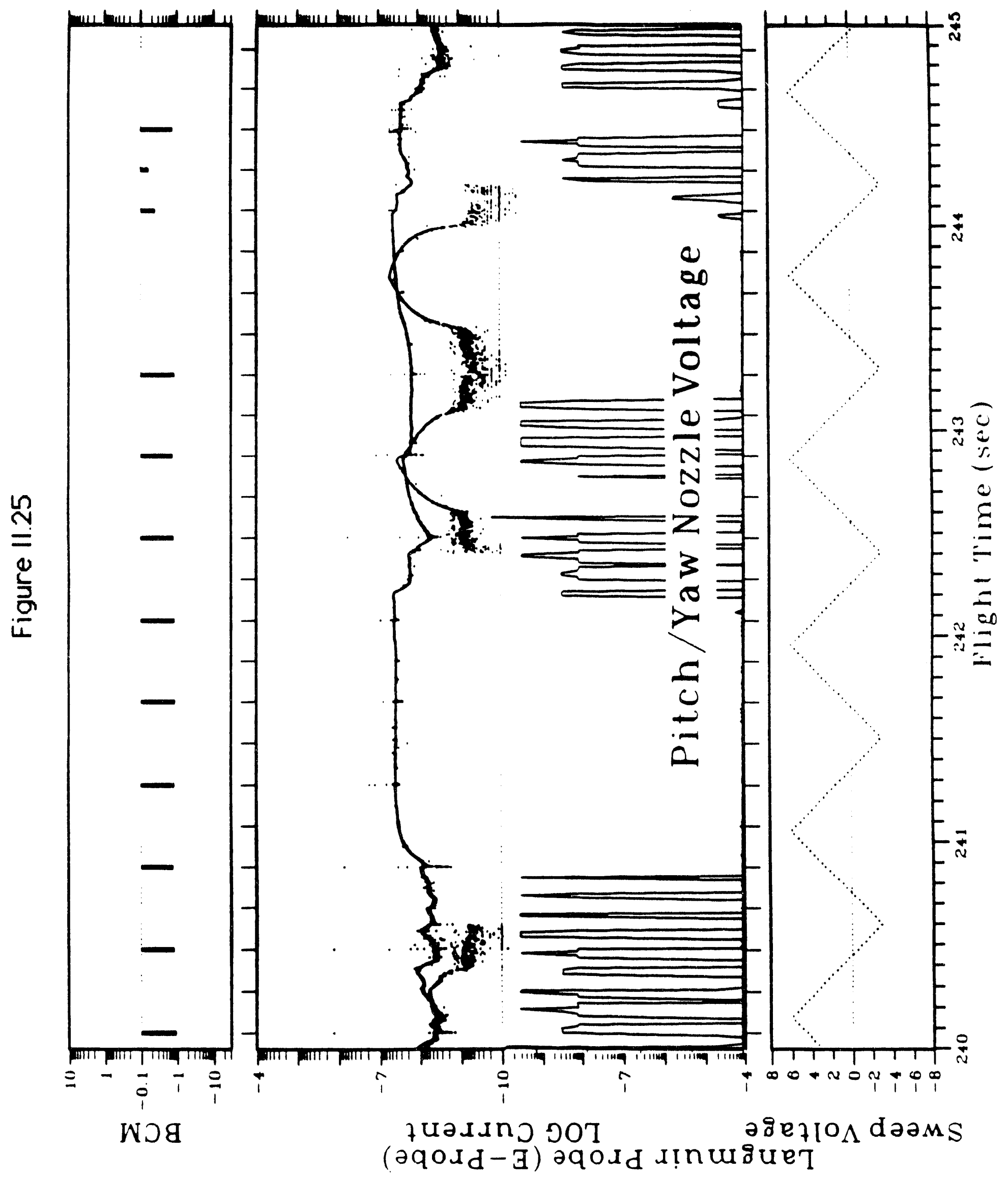


Figure 11.26
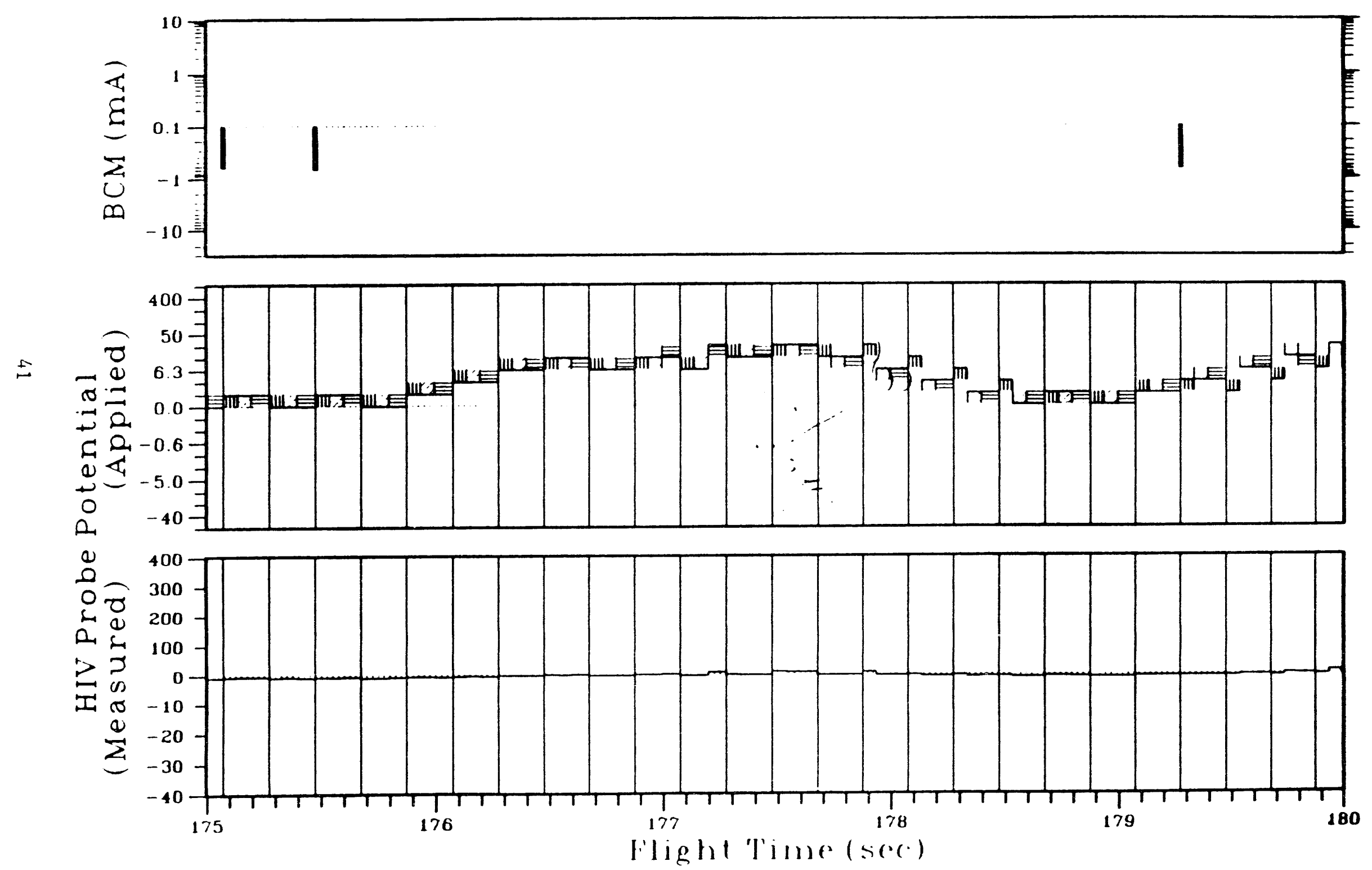
rigure $11 . \angle 1$

APPARENT DENSITY INCREASE DURING OVER-NEUTRALIZATION

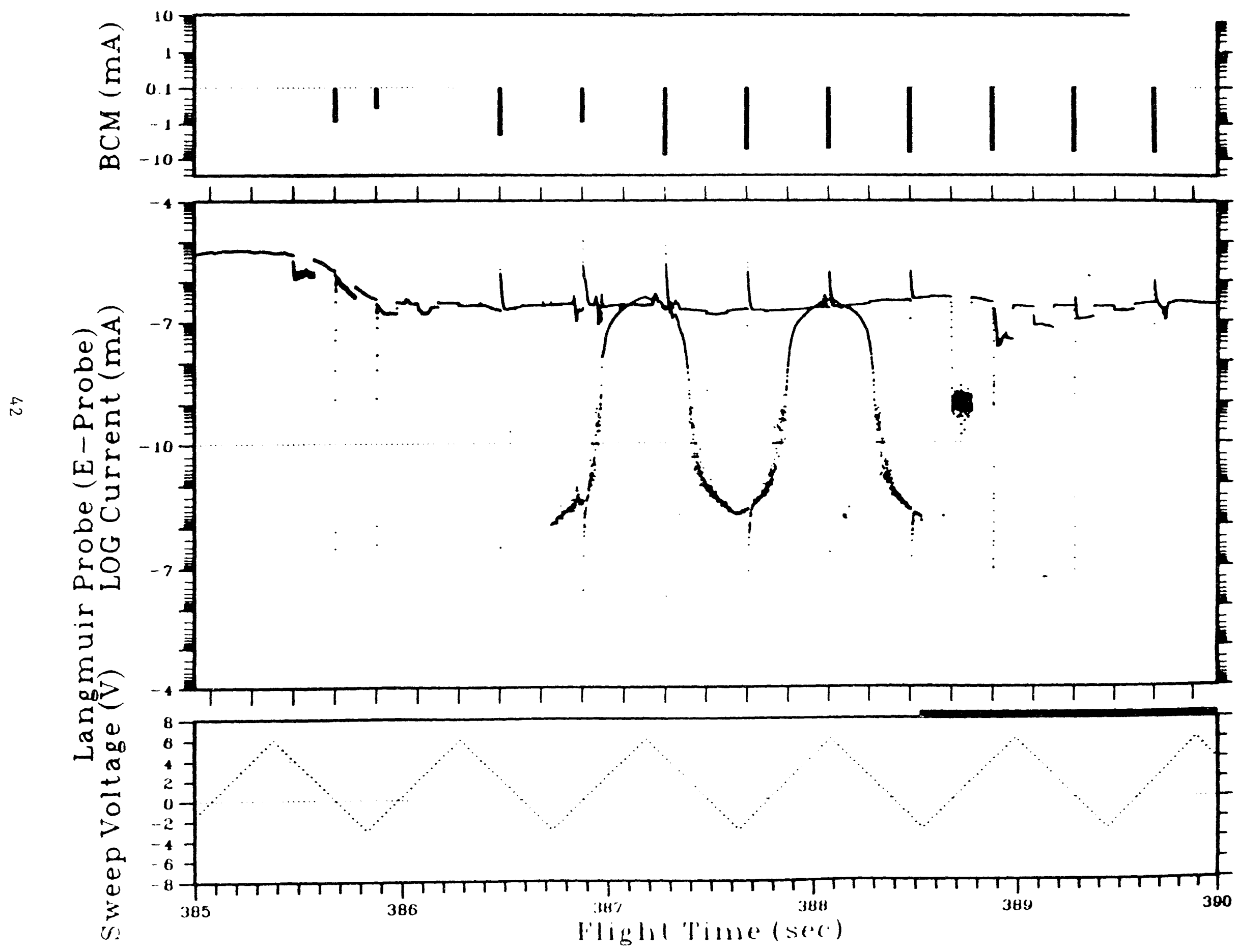




\section{BEAR. IB(E) vs time}

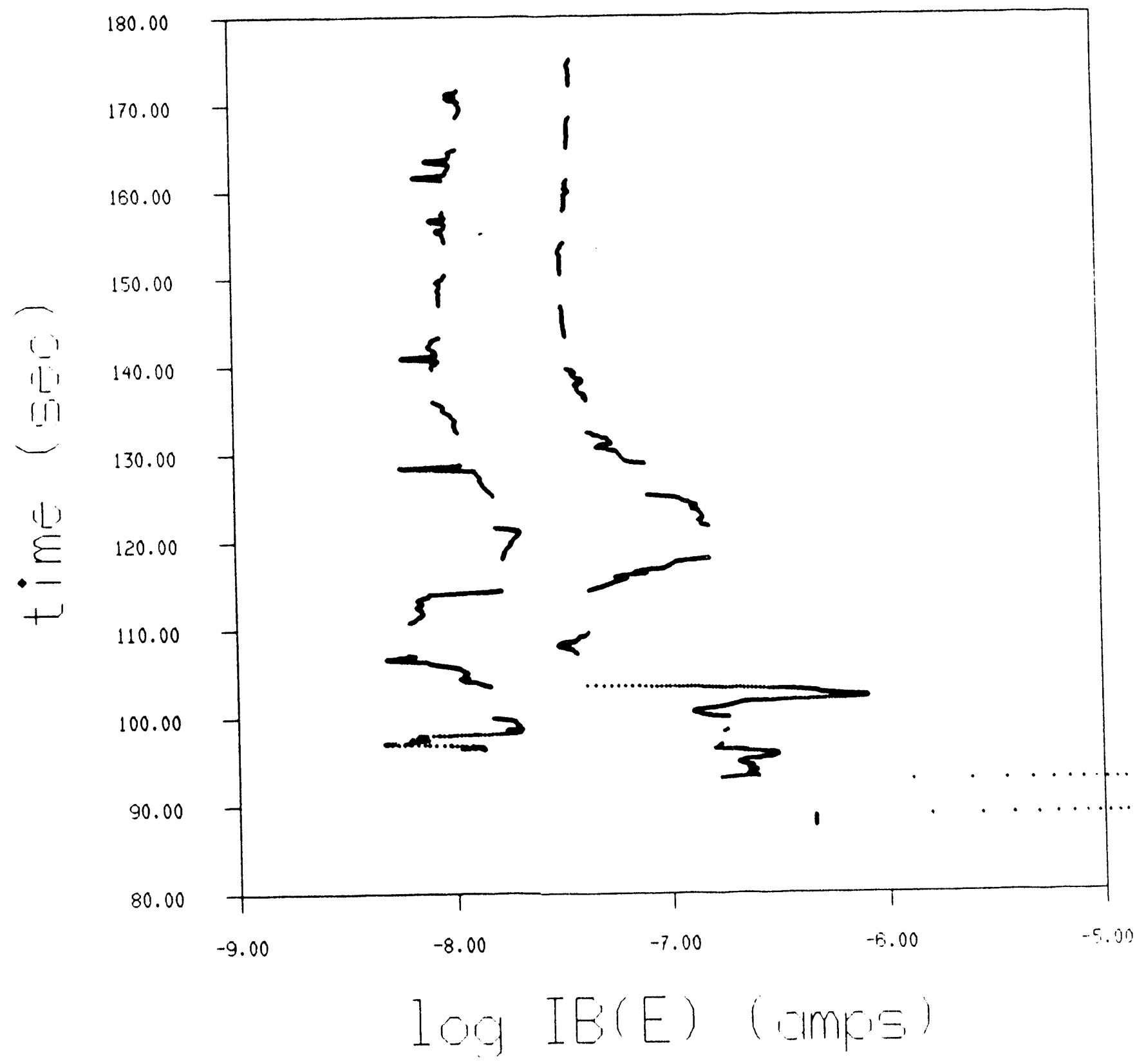

Figure 11.28 


\section{III.1 Design Considerations}

The HIgh Voltage instrument (HIV) was designed to measure the potential of the BEAR vehicle during and after operation of the BEAR neutral beam accelerator. Since the beam emitted from the accelerator contains unequal amounts of $\mathrm{H}^{+}$and $\mathrm{H}^{-}$ions, in addition to the neutral $\mathrm{H}$ particles, initial studies on spacecraft charging of the BEAR vehicle indicated that the vehicle could charge to potentials as high as $1 \mathrm{kV}$ during the $50 \mathrm{\mu s}$ of accelerator operation. Since this potential must be neutralized by return currents from the ionosphere, whose magnitude is highly dependent upon the plasma density, magnetic field configuration, and vehicle-to-plasma potential, measurements of the BEAR vehicle potential were also made 60 and $120 \mathrm{~ms}$ after firing the beam to determine the rate of charge neutralization and to determine if the vehicle accumulated any net charge and potential after multiple beam firings. Thus, the instrument had to be designed to measure potentials on the order of $1 \mathrm{kV}$ in less than $50 \mu \mathrm{s}$ while immersed in a low density plasma. Since the time required to charge the input capacity of a high impedance voltmeter to the vehicle potential is longer than the $50 \mathrm{\mu s}$ beam pulse duration, a different approach had to be taken for the BEAR HIV instrument. To eliminate instrument capacity clarging problems, a low impedance probe-current amplifier (ammeter) was configured as a bridge circuit; which, by trial and search, locates the probe voltage at which the collected current changes polarity (and hence, draws approximately zero current from the plasma). Since a probe immersed in a plasma will draw no net current when it is biased to the floating potential, the vehicle to plasma potential is approximately equal to the negative of the bias voltage on the probe when the collected current polarity reverses.

\section{III.2 Instrument Development and Testing}

The HIV instrument consists of a remote probe and main electronics package. The probe is a gold plated cylinder $5.08 \mathrm{~cm}$ long and $5.08 \mathrm{~cm}$ in diameter, mounted on a $2-m$ long insulated boom that was extended pneumatically. The electrometer circuitry was located inside the probe. The main electronics are contained in an iridited aluminum box with dimensions of $11.91 \times 20.32 \times 11.75 \mathrm{~cm}$. The main electronics package and the HIV boom are located within the Telemetry-Physics (TM) section of the BEAR payload.

The HIV instrument measured the potential of the BEAR vehicle with respect to the ambient plasma for vehicle floating potentials between -400 and +40 volts. Three independent current polarity measurements were taken for each 50-us beam pulse. The first measurement was taken $45 \mu \mathrm{s}$ into the beam pulse, the second was taken $60 \mathrm{~ms}$ after the beam pulse and the third was taken $120 \mathrm{~ms}$ after the beam pulse. After each measurement, the probe voltage was stepped to a new value, which depended upon the previously measured current polarity. 
If the previously measured current polarity was negative, the potential applied to the probe was stepped to the next more positive voltage; and if the previously measured current polarity was positive, the potential applied to the probe was stepped to the next more negative voltage. The HIV instrument can step only between adjacent voltage levels. Table 1 gives the logarithmically spaced positive and negative voltages (10 of each) that can be applied to the HIV probe. When an "equilibrium" condition was reached, such that the probe current polarity reversed between successive measurements taken at the same time relative to the beam pulses, the vehicle-to-plasma potential was assumed to lie somewhere between those two voltage steps. Each of the three measurements were designed to be totally independent. That is, three separate pairs of registers store the previous current and probe voltages for each of the three measurements; the probe voltage was stepped between different voltages for each of the three measurements; and each of the three measurements was capable of reaching an "equilibrium" condition with different potentials applied to the probe.

Figure III.1 shows a block diagram of the BEAR HIV electronics. The instrument consists of an electrometer to measure probe current, a high voltage generator to bias the probe, logic to control the bias voltage on the probe, and a telemetry interface. Figure III.2 shows the BEAR HIV electrometer circuit. Since only the polarity of the input current is measured, diodes are placed in a parallel across the electrometer inputs and feedback loop to prevent the electrometer from saturating (and increasing the response time of the circuit) when the input current is large. Since only a $10 \mathrm{pF}$ capacitor and the capacitance of the diodes across the feedback loop have to be charged by the input current with this circuit configuration, the response time of the electrometer is less than $10 \mu \mathrm{s}$. Since the probe bias can be programmed over a range of about $500 \mathrm{v}$ with respect to vehicle ground, the electrometer is optically isolated from the rest of the instrument and is powered by a floating power supply that tracks the probe bias voltage. The electrometer power supply voltages are $\pm 12 \mathrm{v}$, referenced to the probe bias voltage.

The programmable bipolar probe voltage generator consists of two sections, a high voltage amplifier section and a low voltage $( \pm 15 \mathrm{v})$ amplifier section with digitally selectable gain. Different resistors are digitally switched into the low voltage amplifier circuit, allowing the gain of the amplifier to be changed. The gain of the amplifier is $R_{2} / R_{n}$, where $R_{n}$ is the value of the particular resistor switched into the circuit. Thus, the probe voltage generator is just a simple amplifier with programmable gain. The resistor values were chosen such that each voltage step is approximately twice the previous voltage step (logarithmically spaced voltages). By designing the input section of the amplifier to be optically isolated from the high voltage section and by using a floating \pm 12 v power supply (in addition to the +400 and $-40 \mathrm{v}$ power supplies) in the high voltage section, commercially available (low voltage) operational amplifiers and transistors could be used everywhere in the circuit, except for the drive transistors on the output of the amplifier. The high voltage output of the probe voltage generator, in addition to providing the bias voltage for the probe, was compressed to a value between 0.0 and $5.0 \mathrm{volts}$ so that it could be monitored with telemetry. For probe bias voltages between $+400 \mathrm{v}$ and $0.0 \mathrm{v}$, the voltage 
monitor has an output between $0.5 v$ and $2.5 \mathrm{v}$; and for prcbe bias voltages between $0.0 \mathrm{v}$ and $-40 \vee$ the voltage monitor has an output between $2.5 \mathrm{v}$ and $4.5 \mathrm{v}$.

Figure III. 3 shows a block diagram of the timing circuitry in the BEAR HIV instrument. The timing sequence is initiated by receipt of the BEAR gun sync pulse from the beam accelerator section controller. For each gun sync pulse, the circuitry generates the timing necessary to take three measurements, each separated by $60 \mathrm{~ms}$. The sequence multivibrators generate the precise timing signals necessary for each of the three measurements. The output of the "Count-of Three" counter selects the appropriate set latches that are enabled during each of the measurements; thus, keeping the three measurements totally independent. When a particular set cf latches is selected, the values stored within the latches ( 4 bits for probe voltage, 1 bit for probe voltage polarity, and 1 bit for the previous current polarity measurement) are used for selecting the voltage that is applied to the probe (i.e., switching the appropriate gain setting resistor into the probe voltage generator circuit) and are the values read by the telemetry system. Since the gun sync pulses are asynchronous with respect to the plasma diagnostics section telemetry system, the telemetry sample rate of the HIV instrument is $67 \%$ faster ( 25 samples $\sec ^{-1}$ ) than the actual measurement rate ( 15 samples $\mathrm{sec}^{-1}$ ) to ensure that at least one valid sample is acquired by telemetry for each measurement made.

Figure III.4 shows a timing diagram for the pulses generated by the sequence multivibrators. On the trailing edge of the $45 \mu \mathrm{s}$ pulse $(t=45 \mu \mathrm{s})$, the polarity of the measured current is latched into a temporary latch. The digital value for the probe voltage is set to increment or decrement, depending upon the current polarity measurement, on the trailing edge of the 3 us pulse $(t=48 \mu \mathrm{s})$. The digital value for the probe voltage is incremented or decremented on the trailing edge of the $6 \mu \mathrm{s}$ pulse $(t=54 \mu \mathrm{s})$. Since the digital value of the probe voltage is only incremented or decremented, the probe voltage can only increase or decrease by one step between measurements (taken at the same relative time w.r.t the beam pulse). This new digital value for the probe voltage, the probe voltage polarity, and the current polarity measurement are latched into the appropriate set of latches (selected by the "Count-of-Three" counter) on the trailing edge of the $1 \mathrm{~ms}$ pulse $(\mathrm{t}=1.054 \mathrm{~ms})$. The "Count-of-Three" counter is advanced on the leading edge of the $60 \mathrm{~ms}$ pulse $(t=1.054 \mathrm{~ms})$, setting up the instrument for the next

measurement. The probe voltage generator has until the end of the $60 \mathrm{~ms}$ pulse $(t=61.054 \mathrm{~ms})$ to $\mathrm{stabilize}$ before the next $45 \mu \mathrm{s}$ current polarity measuring interval begins. The digital data (probe voltage, probe voltage polarity, and probe current polarity) will be stable during the $60 \mathrm{~ms}$ pulse.

The telemetry interface consists of one serial/digital line (VO1) and one analog line (V02). The digital representation of the voltage applied to the probe ( 4 bits), the polarity of the probe voltage ( 1 bit), and the polarity of the collected current ( 1 bit) are output on the serial/digital line and is sampled by telemetry at a rate of $25 \mathrm{samples} \mathrm{sec}^{-1}$. The compressed output of the high voltage gensiftot is output on the analog line and is sampled by telemetry at a late of "n samples $\sec ^{-1}$. 
III.3 Bench Tests and Calibration

The HIV instrument was bench tested and calibrated by applying different voltages to the probe through a $250 \mathrm{M} \Omega$ resistor. To test proper sequencing and independence of the three measurements, the HIV GSE generated three programmable (and independent) voltage pulses separc ted by $60 \mathrm{~ms}$ and synchronized gun pulse. The HIV instrument was calibrater by applying voltages between -40 and +400 volts and noting the voltage that caused the equilibrium state to change. Table III.1 shows the voltages and equilibrium states.

\section{III.4 Environmental Testing}

The instrument level environmental tests of the HIV instrument consisted of thermal cycling, shock and vibration testing, and vacuum testing. The instrument was cycled between room temperature $\left(25^{\circ} \mathrm{C}\right),-25^{\circ} \mathrm{C}$ and $+65^{\circ} \mathrm{C}$. The instrument was cycled twice, with a 1 hour soak at each temperature. The instrument performed satisfactorily at each temperature.

The vibration testing consisted of a $60 \mathrm{sec}$ randon shake with an amplitude of $0.005 \mathrm{~g}^{2} \mathrm{~Hz}^{-1}$ for frequencies between 40 and $2000 \mathrm{~Hz}$ and an $11-\mathrm{ms}$ 25-g shock. Both shock and vibration tests were performed on all three axes. The instrument was powered on during the shock and vibration testing and functioned correctly during the test and also during subsequent bench testing. Additional shock and vibration testing was performed at AFGL on the telemetry physics section of the payload, and at WSMR on the entice payload. The HIV instrument functioned correctly during all of these subsequent tests also.

Vacuum testing of the instrument was performed at NRL and in the plasma chamber at the University of Maryland Center for Fusion Research to verify operation of the instrument in a vacuum under conditions similar to what would be encountered during the actual flight. Since the HIV instrument contains a high voltage power supply, extensive tests on the prototype HIV instrument were performed in the NRL vacuum chamber to determine the susceptibility of the instrument to damage from arcing of the power supply and to determine the range of densities necessary for arcing of the power supply to occur. The results of these tests were: 1) The densities necessary for arcing to occur in the power supply were much higher than the expected density during the operational portion of the BEAR (1ight. 2) The opto-coupler in the high voltage generator was susceptible to damage from arcing, but only after being exposed to arcing for an extended peisod of time (30) to 60) minutes). As a result of these tests, protection diodes were installed in the flight instrument to make the circuits less susceptible to damage from atcing.

Figure III.5 shows a schematic of the chamber in which the plasma environmental testing was performed at lh University of Maryland and Figure III.6 is a representation of the experimrntal mrangement at the site. What is notable about the chamber setup of Figun. III.' is that in addition to a 
magnetic field produced by large Helmholtz coils, there is also a plasma source and plasma guide fields at the left end of the Figure. This allowed us to produce varlable density plasmas; in addition, it allowed us to cancel the effect of the earth's magnetic field by using the central core field. We were able to vary the neutral pressure over two orders of magnitude but operated most consistently in the range near $2 \times 10^{-5}$ Torr. Diagnostic instrumentation ranged from a series of ion gauges and controllers to digitized sweeps of a spherical Langmuir probe. A/D conversion allowed us to determine electron density and temperature at the approximate site of the sensor element with a high degree of repeatability.

From Figure III.6, it can be seen that the instrument was operated outside the plasma chamber except for the sensor which was suspended by its leads from approximate chamber center. There were four leads, as shown, which were led through a sealed connector in the chamber wall. Those leads, in addition to the electronics box and measurement instrumentation, had voltages to the 500 volt level during testing. The test voltage was connected to the instrument as shown and the return was connected to laboratory ground (i.e., the chamber wall).

The primary results of the experimentation are shown in Figures III.7 through III.9. Figure III.7 shows one set of voltage steps applied during the three pulsing intervals shown in the lower left of Figure III.6 (Note that the pertod of the 50 usec pulse $\left(V_{1}\right)$ is exaggerated on this scale). As covered above, we made three measurements during the $0.2 \mathrm{sec}$ duty cycle: one during the pulse, a second one at $60 \mathrm{msec}$ after, and a third following by another 60 msec.

Figures III.8 and III.9 are the results of measurements made versus voltage applied during the first step (i.e., within 50) usec). These are the most relevant results for the BEAR rocket as the pulsing time interval is most critical. Since the instrument searches between two levels as described above, the measurement resolution is a function of the level of applied voltage, voltage levels differing by approximately a factor of 2 . Hence during the application of $-300,-400$, and -500 volts the instrument reads its maximum range which is an approximate level between -200 and -400 volts. Note that application of a negative voltage to the sensor is equivalent to a positive vehicle-to-sensor potential (or in this case chamber-to-sensor potential)

Results of the testing verified that the instrument searched correctly for densities in the range $1 \times 10^{3}$ to $1 \times 10^{7} \mathrm{~cm}^{-3}$. Electron temperature as measured by a diagnostic Langmuir probe sphere also suspended from the chamber was usually measured near 4-5 ev, higher than expected ionospheric densities. This temperature is difficult to control in the hot filament source not only because the electrons are driven from the filament with an accelerating potential but that they also diffuse inward to the chamber area guided by the axial magnetic field.

III.5 Integration 
Integration consisted of installing the HIV instrument into the payload and verifying operation of the instrument and interface between the experiment and payload. Except for a timing problem on the serial/digital line, the HIV instrument functioned correctly during integration.

\section{III.6 Flight Performance and Data}

The HIV instrument performed as expected throughout the BEAR flight. The HIV was turned on $122 \mathrm{sec}$ after liftoff and continued to return valid data unt 11 the HIV boom was retracted and then efected from the vehicle at 390 and $401 \mathrm{sec}$ respectively. In addition to providing information on vehicle charging during the different modes of accelerator operation, the HIV provided information on the effects of thruster firings on the vehicle potential.

\section{Vehicle Charging Observations}

The top panel of Figure III.10 shows the net accelerator beam current and the time of firing for the beam, during accelerator operation with the neutralizer on. Approximately $1 \mathrm{~mA}$ of negative current is observed whenever the beam successfully fires. The HIV digital data is shown in the next panel. The HIV sample taken during the beam firing (Sample \#1) is shown as the data point immediately following the BEAR gun sync pulse (the thin vertical line through the panel). The HIV samples taken $60 \mathrm{~ms}$ and $120 \mathrm{~ms}$ after the BEAR gun sync pulse (Sample \#2 and Sample \#3) are shown sequentially following Sample $\# 1$. Whenever the accelerator fired, the HIV instrument indicated that the vehicle potential was more positive during Sample \#1, than the potential applied to the HIV probe. Since the applied probe potential can step by only one voltage level for each sample, the HIV probe potential gradually steps more negative after each accelerator firing (implying that the vehicle has charged positive), as the instrument searches for the applied potential that causes the current polarity to reverse. From approximately 271.5 to 274.0 $\mathrm{sec}$, the applied probe potential does not step to more negative values because the accelerator was firing only every other time. From 274.0 to 274.5 , three consecutive beam firings occur and the applied potential steps more negative. From 276.0 to $277.3 \mathrm{sec}$ no beam firings occur and the applied probe potential steps back to zero volts. The third and fourth panels show the Langmuir probe current and sweep voltage. The large decrease in Langmuir probe electron saturation current and the stepping of HIV Samples \#2 and \#3 to a positive applied potential during the interval from 272.0 to $274.0 \mathrm{sec}$ is caused by vehicle thruster firings.

Figure III.11 shows the response of the HIV instrument when the beam is over neutralized. The current polarity of Sample \#1 (measured during firing of the beam) indicates that the vehicle is charging to a negative potential, as would be expected with a net positive beam current. Also, in absence of thruster firings, the applied probe potential during Sample \#2 and \#3 approaches zero volts, indicating that the charge and potential on the vehicle is neutralized in a time interval hotel than $50 \mathrm{~ms}$. 


\section{The Effects of Vehicle Thruster Firings}

Figure III.12 shows the effect of nearly continuous thruster firings on the HIV and Langmulr probe data. During thruster firings, the HIV applied probe potential steps to approximately 25 volts. When the thruster firings cease, the applied probe potential steps back to zero volts. Large decreases and fluctuations in the Langmuir probe electron saturation current and the absence of an ion current for negative Langmuir probe sweep voltages are also observed during vehicle thruster firings.

\section{Summary of HIV Observations}

(i) The HIV indicates that the BEAR vehicle is charged to a positive potential during the beam pulse when the net beam current is negative, and charged to a negative potential during the beam pulse when the net current is positive.

(ii) In the absence of thruster firings, the applied potential during Samples \#2 and \#3 approaches zero volts, implying that the charge and potential on the vehicle is completely neutralized within $60 \mathrm{~ms}$ of the beam pulse.

(iii) During vehicle thruster firings, the applied probe potential typically steps to approximately 25 volts. The overall effects of thruster firings on vehicle charging requires further study. 
Table III.1: HIV Probe Potentials

\begin{tabular}{cc} 
D/A Input (Binary) & Probe Potential \\
\cline { 2 - 2 } 11001 & $360.0 \mathrm{~V}$ \\
11000 & $175.0 \mathrm{~V}$ \\
10111 & $91.0 \mathrm{~V}$ \\
10110 & $45.0 \mathrm{~V}$ \\
10101 & $23.0 \mathrm{~V}$ \\
10100 & $12.5 \mathrm{~V}$ \\
10011 & $6.5 \mathrm{~V}$ \\
10010 & $3.0 \mathrm{~V}$ \\
10001 & $1.5 \mathrm{~V}$ \\
10000 & $-0.3 \mathrm{~V}$ \\
00000 & $-0.3 \mathrm{~V}$ \\
00001 & $-0.4 \mathrm{~V}$ \\
00010 & $-0.7 \mathrm{~V}$ \\
00011 & $-1.1 \mathrm{~V}$ \\
00100 & $-1.5 \mathrm{~V}$ \\
00101 & $-2.6 \mathrm{~V}$ \\
00110 & $-5.0 \mathrm{~V}$ \\
00111 & $-9.5 \mathrm{~V}$ \\
01000 & $-19.0 \mathrm{~V}$ \\
01001 & $-39.0 \mathrm{~V}$
\end{tabular}




\section{Nrl High Voltage Instrument}

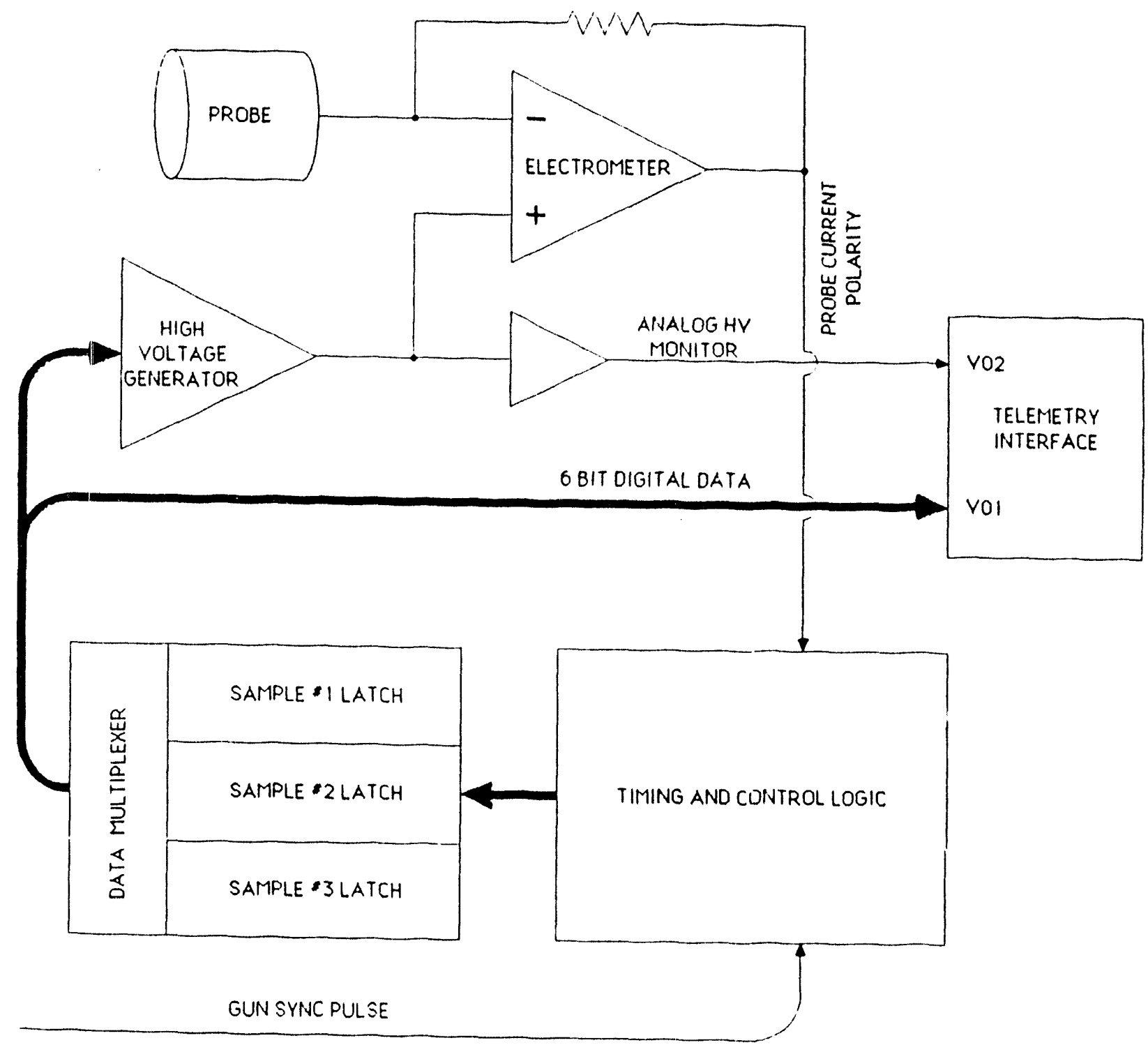

Figure III.1 


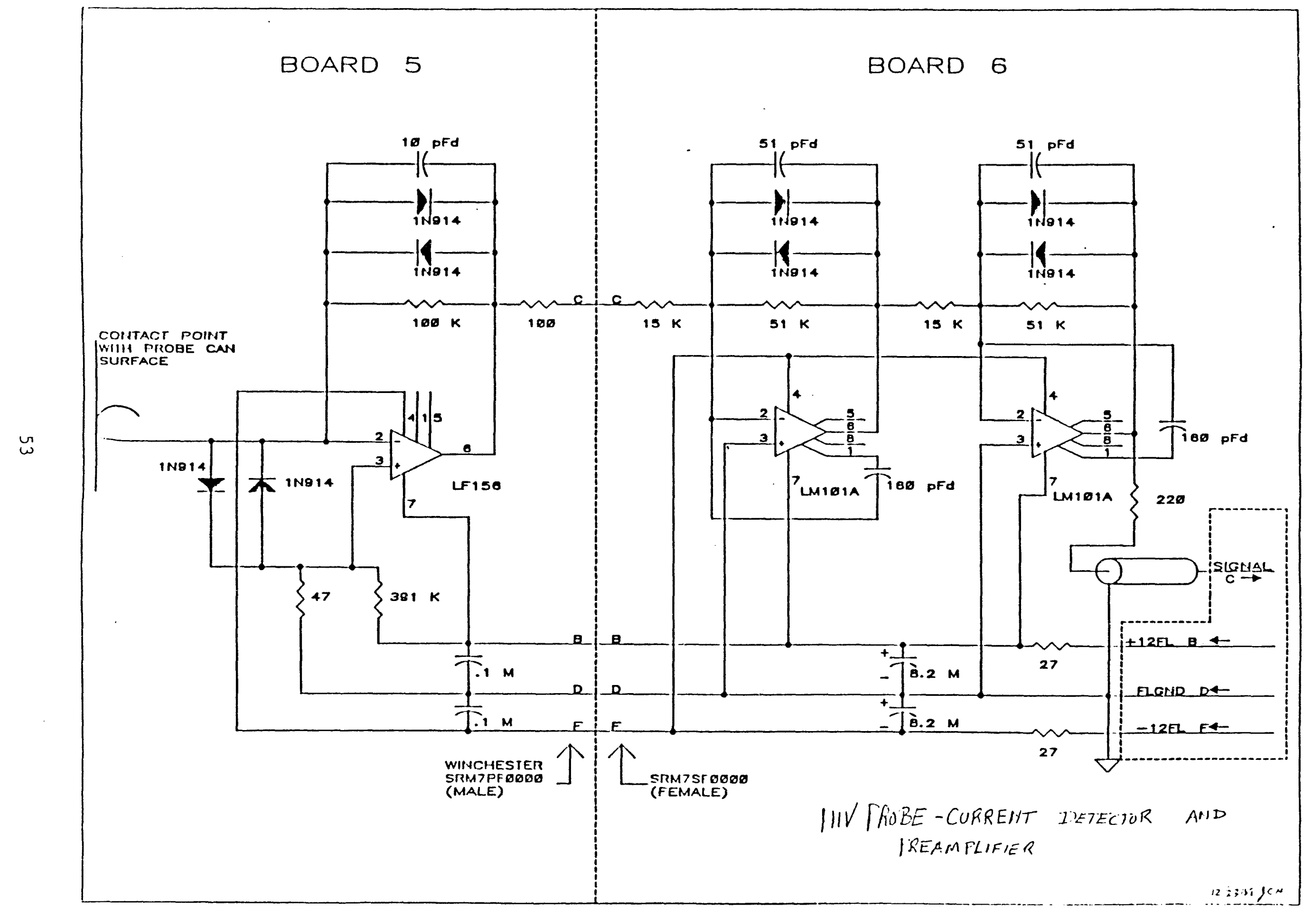

F1gure III.2 


\section{HIV TIMING SEQUENCE GENERATOR}

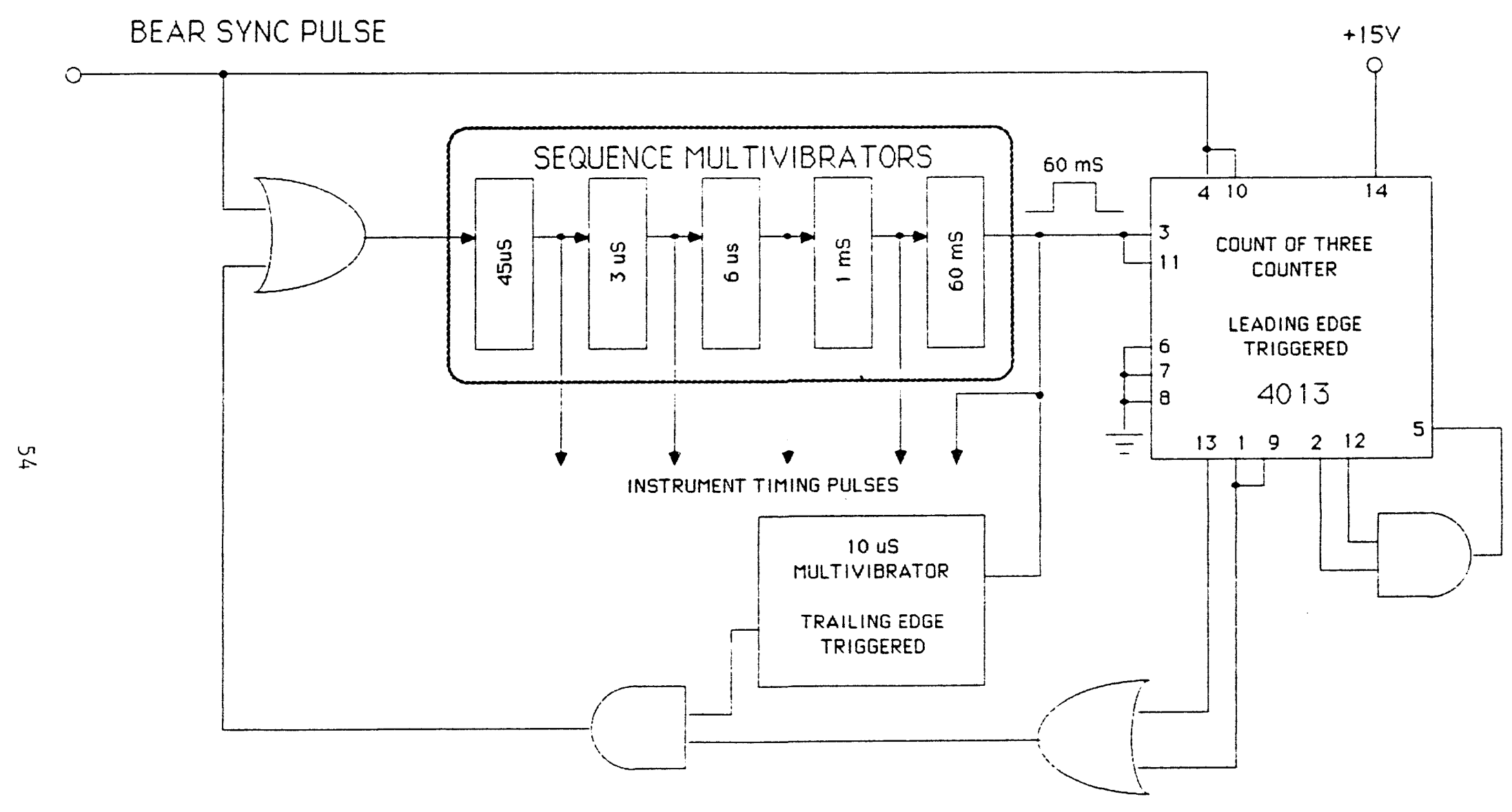

Figure III.3 


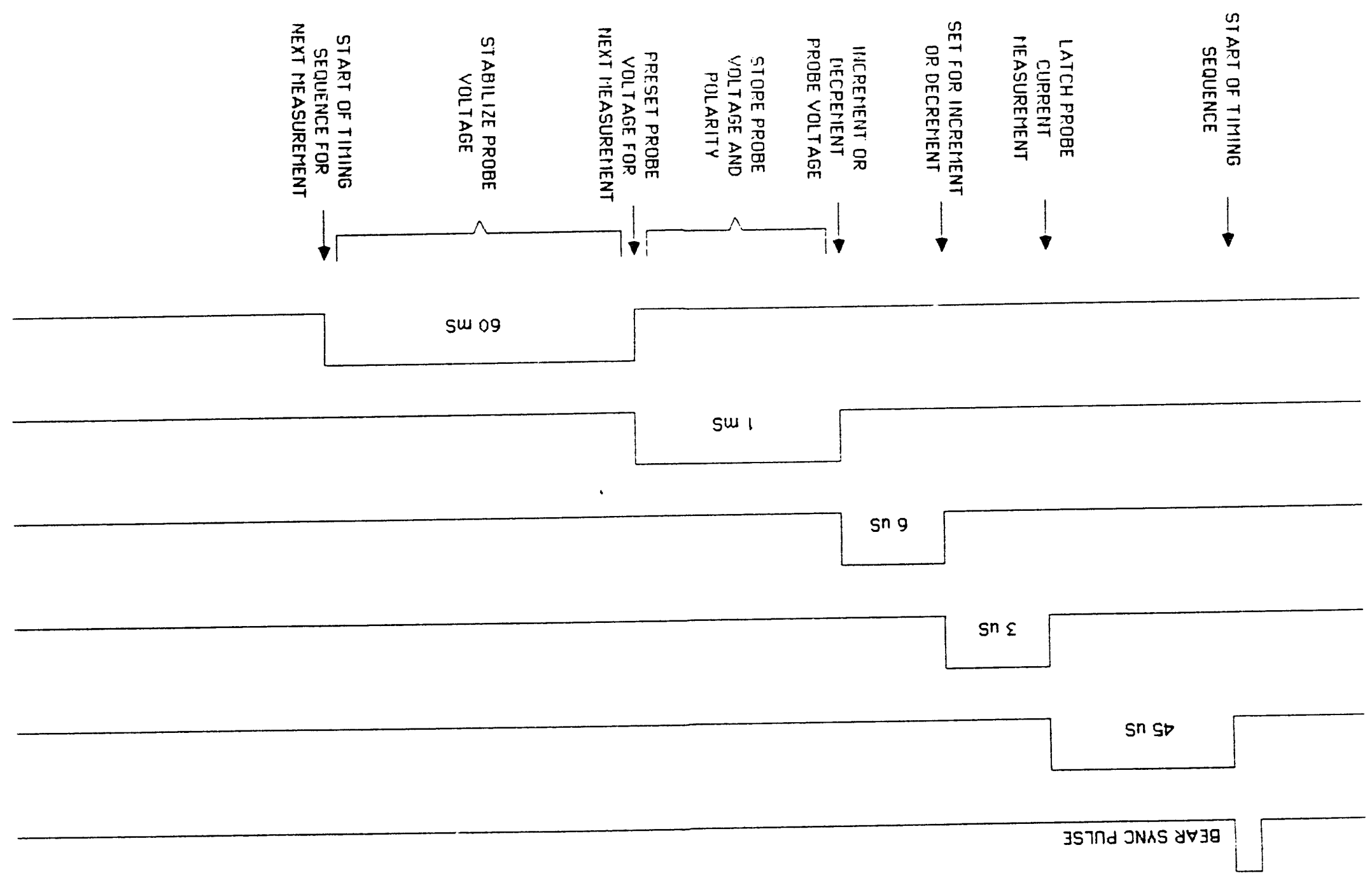

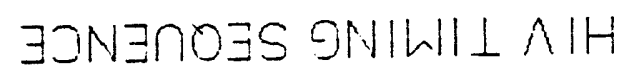




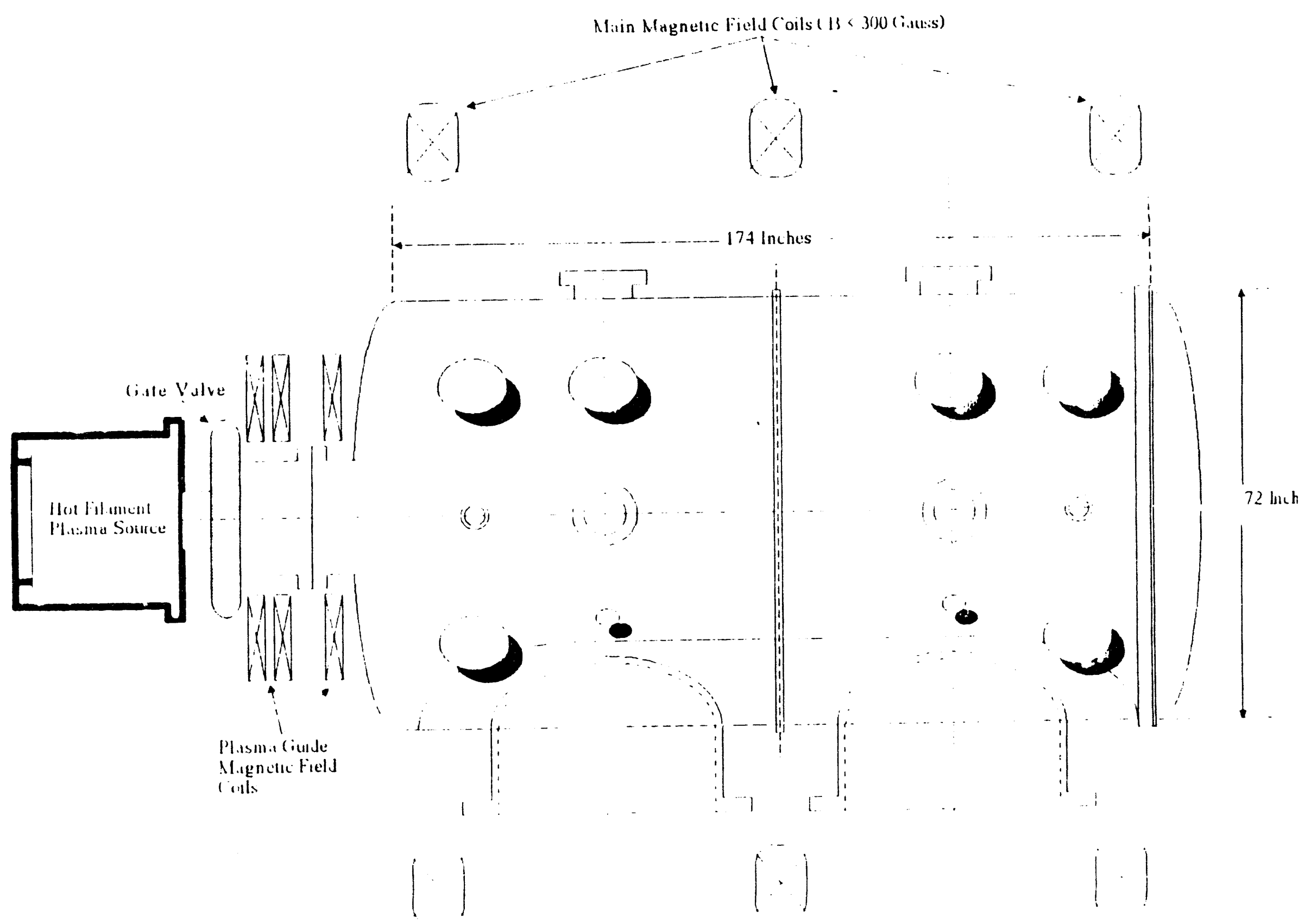




\section{UNIVERSITY OF MARYLAND LARGE VACUUM CHAMBER HIV TESTING CONFIGURATION}

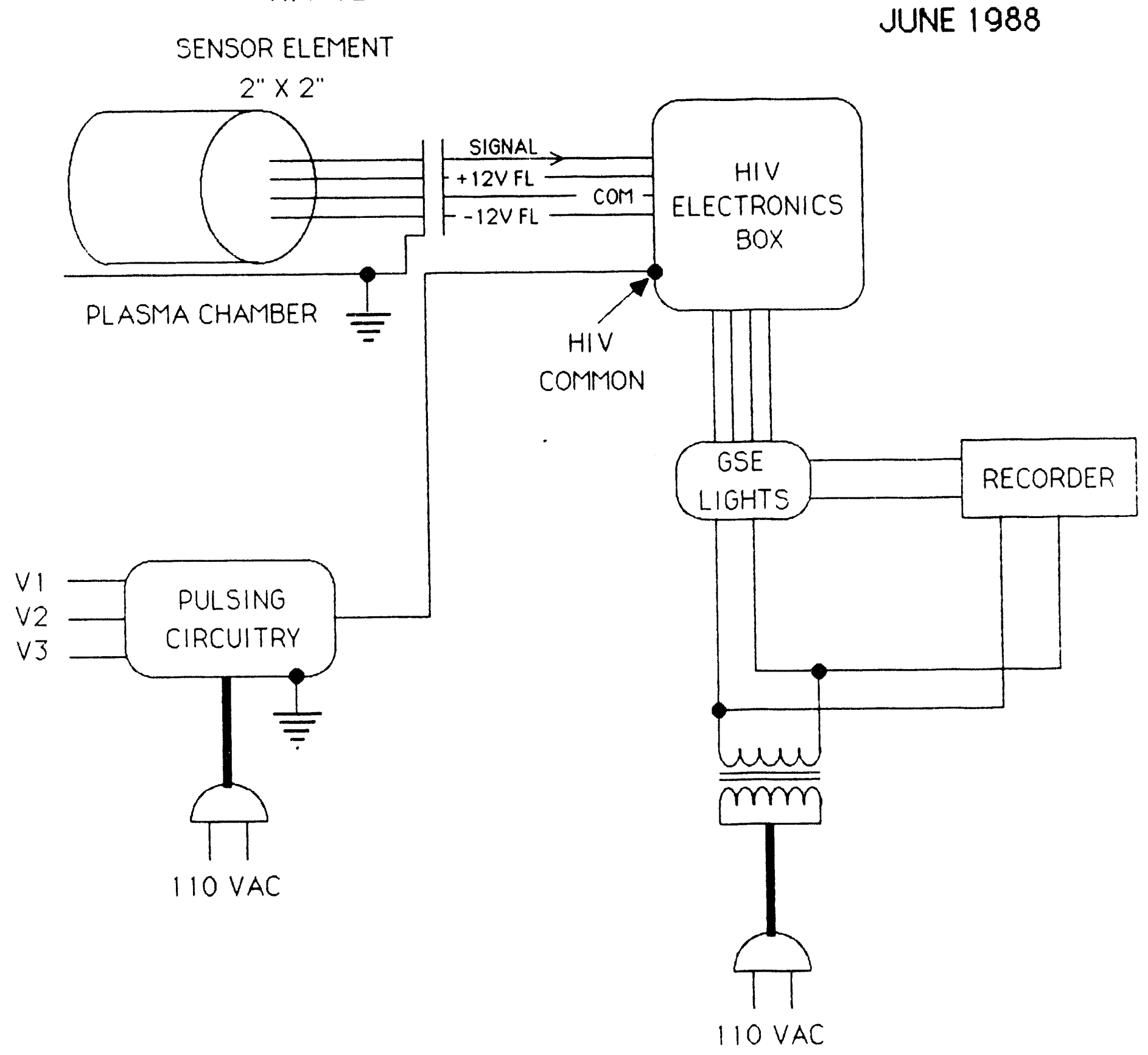

Figure III.6 


\section{APPLIED SENSOR VOLTAGE (VOLTS)}

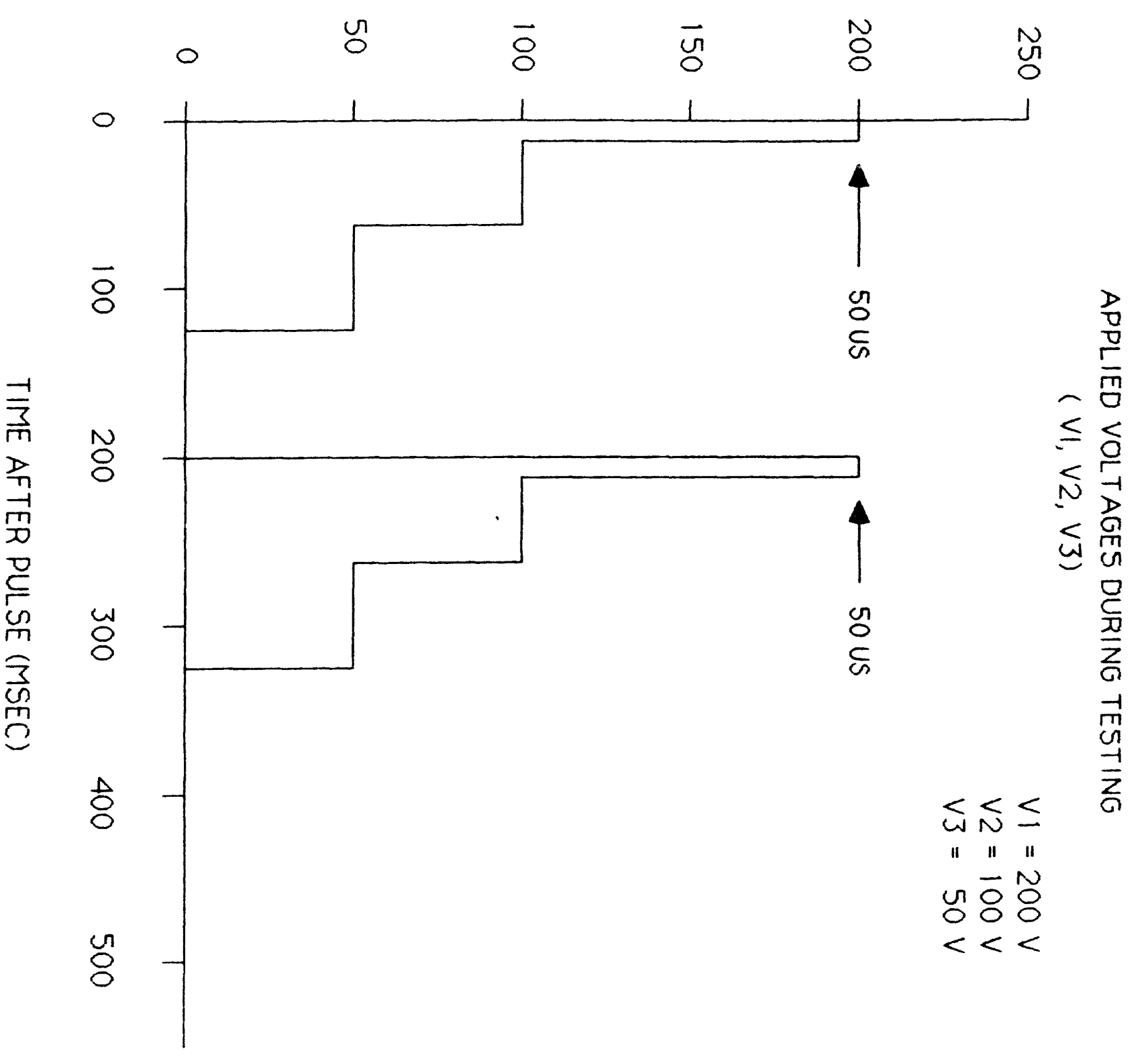


HIV DETECTOR VOLTAGE RANGE (VOLTS)

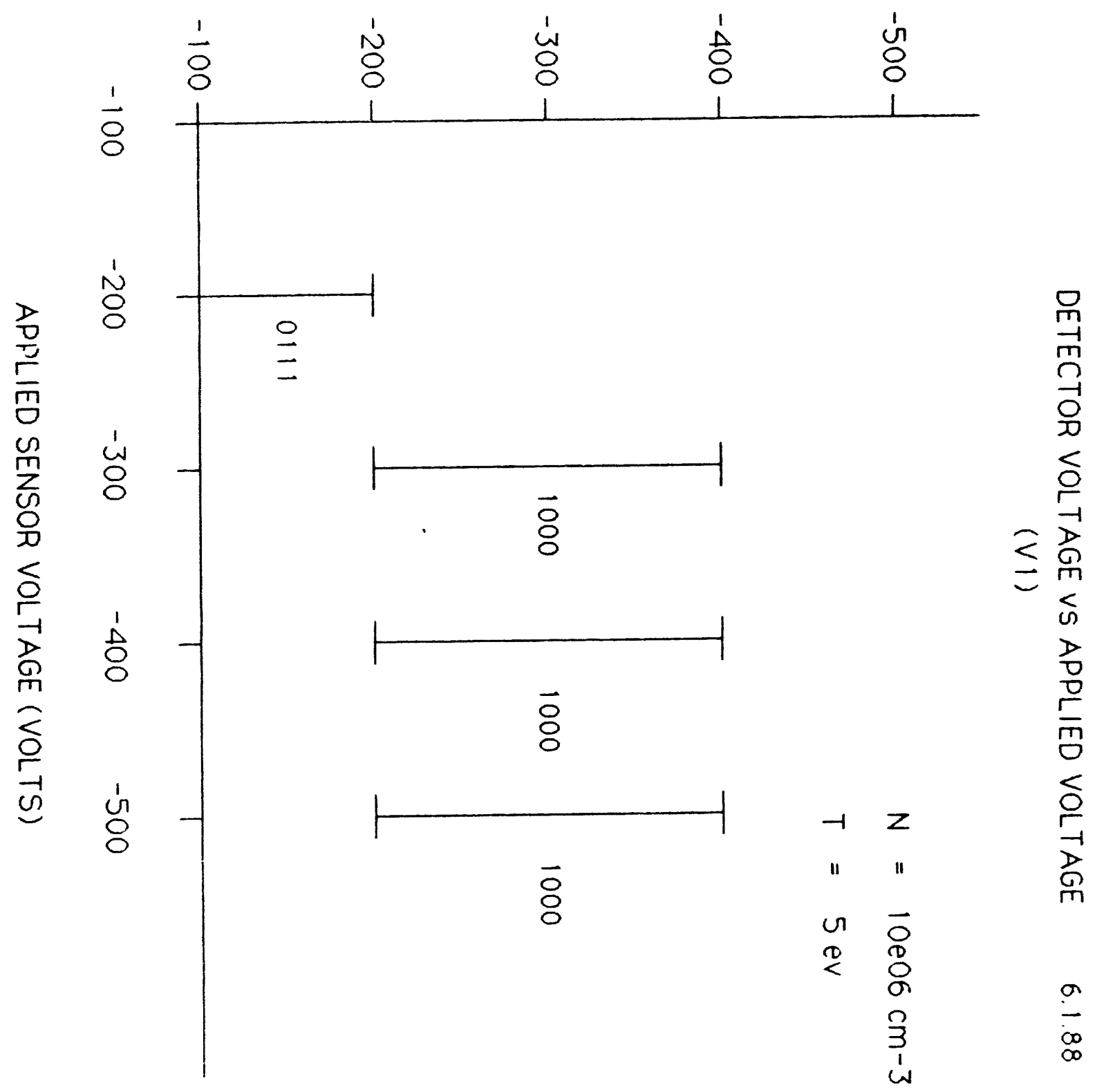




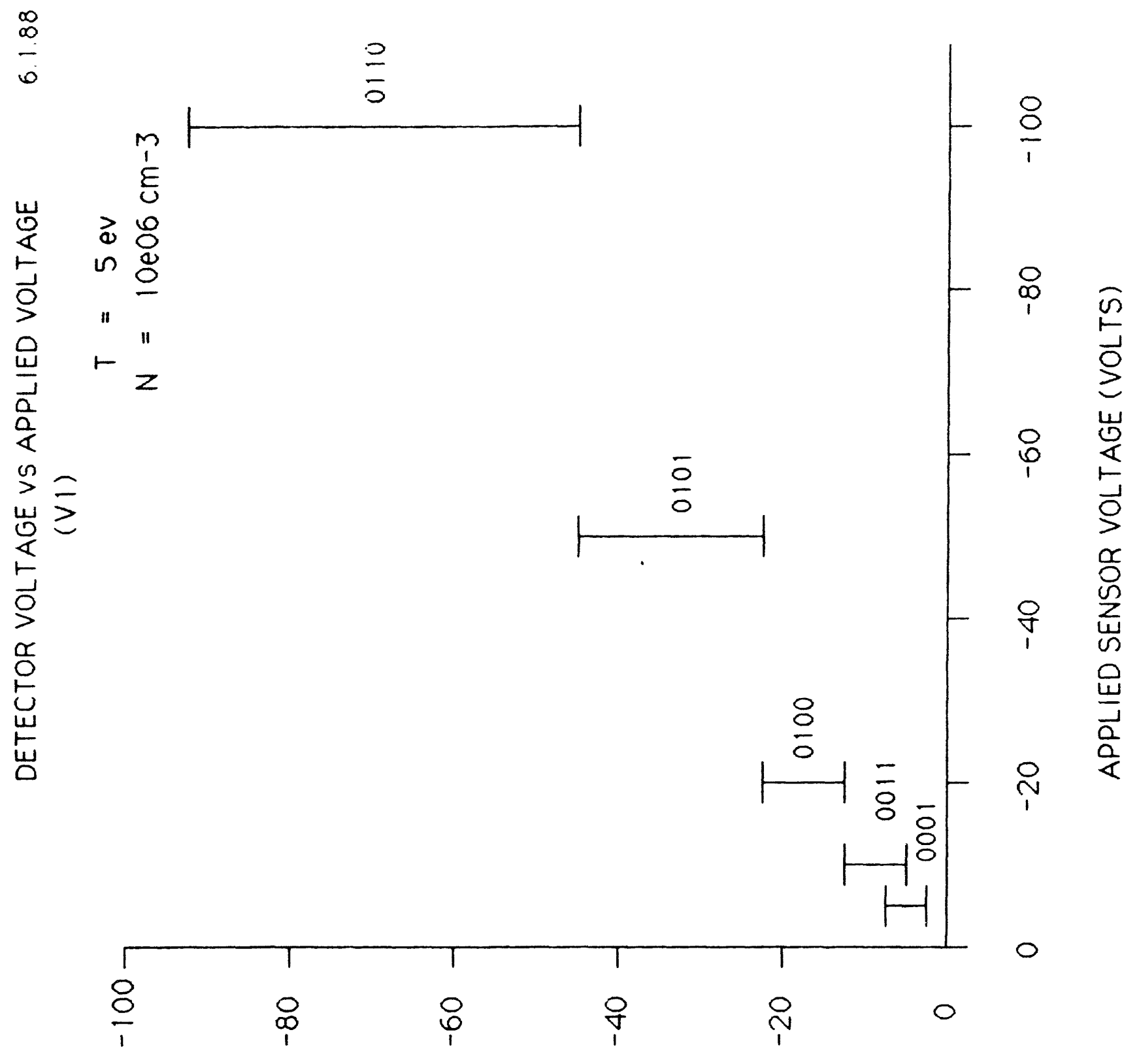

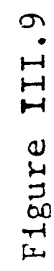

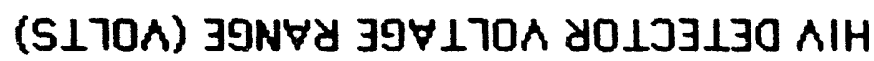



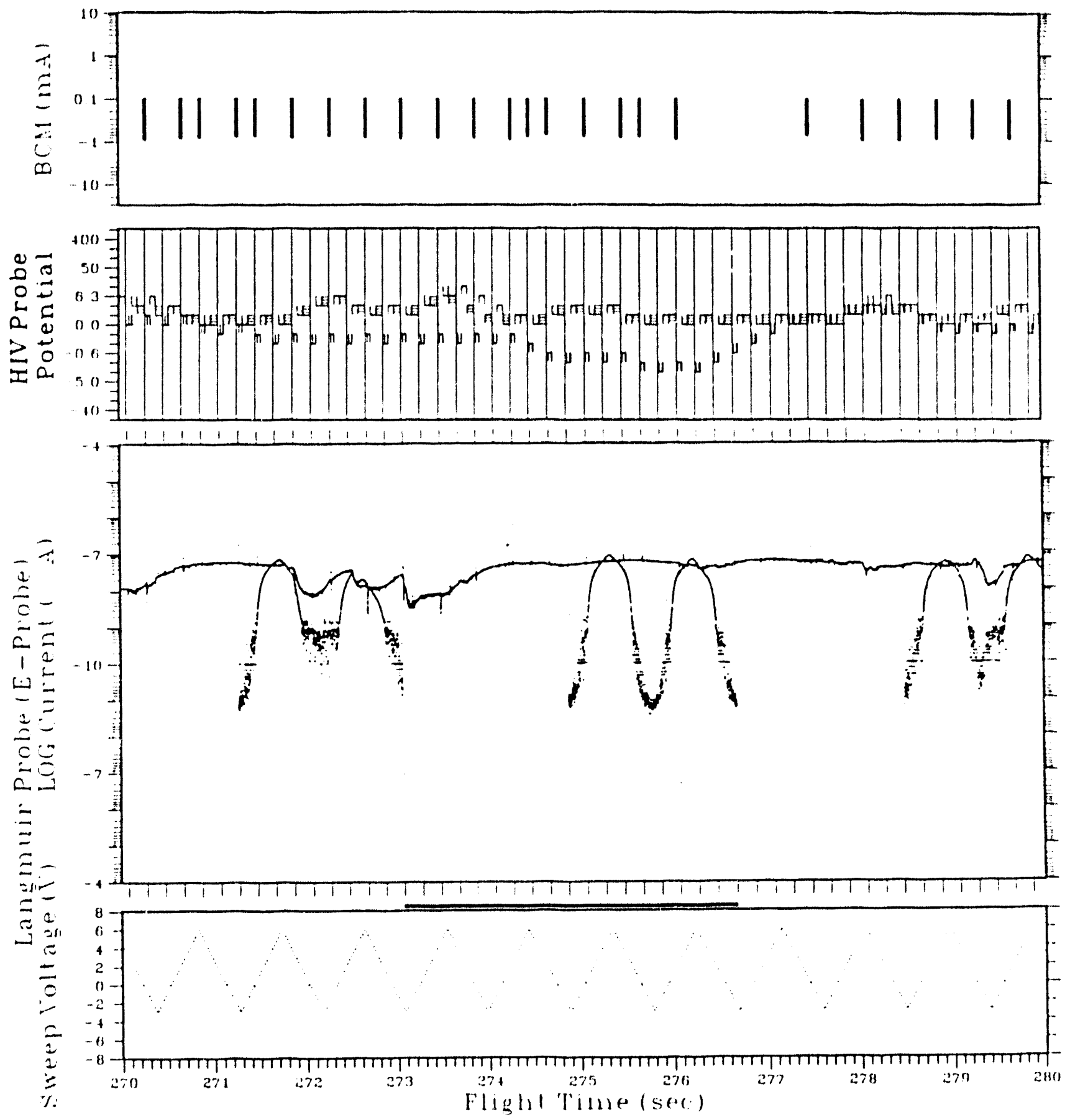

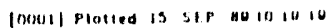

F1gure III.10 

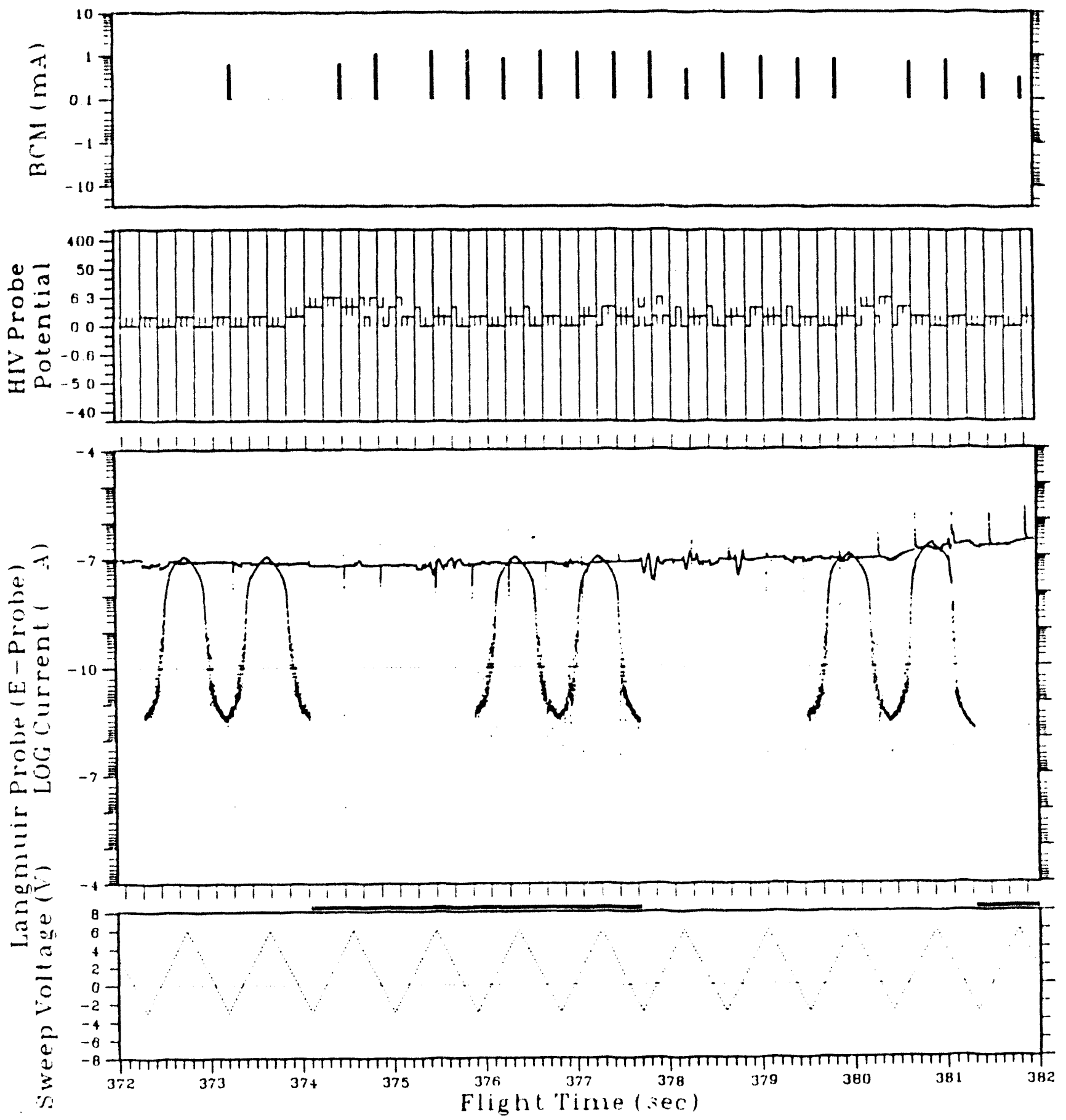

100011 riolled 19-256-10 10,202

Figure III.11 

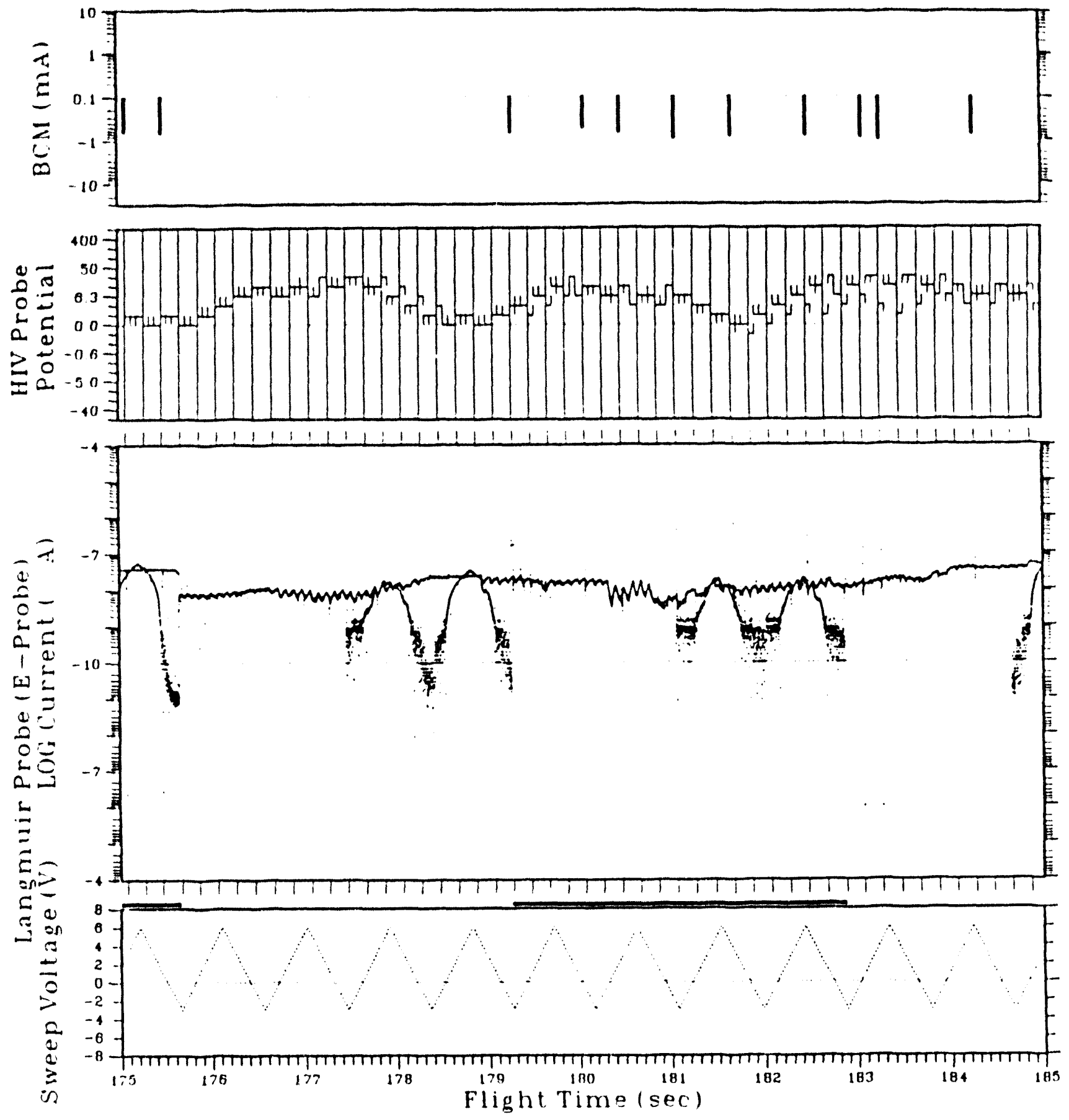

[0003) Plotied 19-3ep-80 140010

F1gure III.12 


\section{PLASMA WAVE RECEIVER}

\section{IV.1 Design Considerations}

The Plasma Wave Recelver (PWR) on the BEAR payload was designed to be a spectrum analyzer of plasma waves and plasma turbulence generated in the Ionosphere by operation of the BEAR neutral beam accelerator. The plasma wave spectrum near the BEAR accelerator will provide significant diagnostic information of the effects the tonosphere has on the propagation of the neutral beam. The range of wave frequencies expected for the BEAR experiment are shown schematically in Figure IV.1.

Positive and negative ions are normally emitted along with the BEAR neutral beam particles. It is reasonable to expect that pulsing of the neutral beam would produce charging of the payload and stimulate ionospheric plasma turbulence. The BEAR experiment can also be expected to demonstrate beam-plasma interactions similar to those seen in previous rocket and Shuttle charged particle experiments. These issues are of particular importance to the BEAR program because beam-plasma interactions can lead to anomalous electron heating and subsequent enhanced ionization of neutral beam particles, effectively stopping propagation of the beam. Similar instabilities may arise if the payload is charged to high negative potentials due to net positive charge emission. Initial model studies of spacecraft charging indicated that potentials as high as $1 \mathrm{kV}$ were possible. Under these conditions, return currents in the ionosphere would lead to instabilities which would produce a spectrum of plasma wave turbulence. Various descriptions [Gary, Borovsky, Pongratz] of plasma waves and turbulence have suggested that both high frequency (electron plasma oscillations) and low frequency waves (ion waves) are likely to occur. In particular, if the return current from the ionosphere to the charged payload supports electron currents, then lower-hybrid waves and whistler waves below about $20 \mathrm{kHz}$ are expected.

The pulsed operation of the BEAR beam resembles a mini-lightning stroke in which the $\mathrm{H}+$ and $\mathrm{H}$ - ions carry an electrical current along with the effective neutral current of $H$ atoms in the beam. Ion-ion instabilities, where the beam-associated ions are drifting through the background ions can lead to wave growth at a fraction of the ambient ion plasma frequency. Such waves would be Doppler shifted to frequencies below about $1 \mathrm{MHz}$ in the spacecraft flame. Electron-ion instabilities can produce plasma waves at higher frequencies, up to $50 \mathrm{MHz}$ in the spacecraft frame. However, ambient plasma waves are also expected, at the lower frequencies of the ion and electron gyrofrequencies, in the 10 $\mathrm{Hz}$ to $1 \mathrm{MHz}$ range. Coincidentally, the $5 \mathrm{~Hz}$ pulsing rate (200 $\mathrm{ms}$ between 50-usec pulses) of the BEAR neutral beam is neat the ion acoustic wave frequency for the ambient NO+ ions and conld provide an additional coupling mechanism for the generation of llasma fluctuations about the payload. 
Plasma waves in a magnetized environment like the ionosphere tend to become polarized with respect to the magnetic field direction. Wave propagation either parallel or perpendicular to the the magnetic fleld, along with the observed frequency, provides an important diagnostic to identify the generation mechanism for the wave. Electron plasma oscillations tend to be field-aligned as do ion acoustic waves resulting from fleld-aligned gradient instabilities such as may occur if payload charging requires electron return currents; however, plasma waves in the same frequency range can also assume perpendicular polarization (becoming Bernstein modes) if driven by cross-field currents. It is therefore important to provide for sampling of the plasma wave spectrum both perpendicular and parallel to the earth's magnetic field. The coupling between beam particles and plasma wave modes is also dependent upon the propagation direction. Thus the payload was oriented parallel to the magnetic field for a portion of the flight and later tipped to be perpendicular to the field.

The initial frequency estimates above ranged from $\mathrm{DC}$ to $100 \mathrm{MHz}$, covering the lowest ion frequencies $(\sim \mathrm{Hz})$ up to the electron plasma frequency associated with electrons that might be emitted during the beam firing (75-100 $\mathrm{MHz})$. Later estimates lowered the upper frequency limit to about $50 \mathrm{MHz}$. The 50 -usec length of the neutral beam pulses implies than an instrumental frequency resolution well above $20 \mathrm{kHz}$ is required. Based on such considerations, the PWR was designed as a set of stacked receivers covering separate frequency ranges, each receiver being designed to provide optimum frequency resolution consistent with the telemetry bandwidth available. Below $1 \mathrm{MHz}$, the PWR consists of several waveform analyzers: the DC/VLF and Baseband (BB) receivers. Standard telemetry systems restrict waveform measurements to a maximum bandwidth of about $100 \mathrm{kHz}$. However, in the BEAR experiment the frequency range near $1 \mathrm{MHz}$ was expected to contain ambient electron plasma oscillations which could be crucial to proper diagnosis of beam pulse interaction and propagation effects. Therefore, a separate dedicated telemetry link was provided for the Baseband receiver and modulated by direct-FM techniques to provide a 1-MHz bandwidth. This design made the telemetry system effectively part of the Baseband receiver design. Waveform sampling by the DC/VLF, and BB receivers throughout the BEAR flight thus allows us to acquire the maximum resolution of the spectrum possible.

Above $1 \mathrm{MHz}$, waveform sampling is no longer possible with the telemetry bandwidths available, so Stepped Frequency Receivers (SFRs) are used to sample the signal strength in discrete $30 \mathrm{kHz}$ bandwidths using the heterodyne technique. For these measurements, spectral resolution depends on the stepping rate (dwell time) and the step size. The stepping rate was maximized consistent with the required dwell time for the receiver bandwidth used, and with the desired goal of cycling each SFR through all its frequency steps between the 50-usec beam pulses. Each SFR covers about an octave in frequency with 38 steps per stepping cycle; the dwell time at each sten is $20 \mathrm{~ms}$. The stepping rate is also designed to allow each 50-usec beam pulse to occur at a different frequency step in each cycle. Ten SFRs are used to cover frequencies from about $0.8-50 \mathrm{MHz}$, and all the SFRs are stepped together 
in their respective frequency bands. At each $20 \mathrm{~ms}$ step of an SFR four measurements of the signal strength are acquired: the maximum, average, and minimum amplitudes, and the gain setting of the receiver for that step. Figure IV.2 shows the frequency ranges of the PWR in terms of the bandwidths or stepping intervals of the individual receivers.

The signal inputs to the PWR receivers are provided by sensors mounted on the tips of two mutually opposing Weitzmann booms which are deployed from the payload at about $100 \mathrm{~km}$ altitude. The tip-to-tip length of the booms is about 6 meters, and forms the baseline for electric field measurements. The PWR electric field measurement is a differential measurement between two high input-impedance preamplifiers in boom-tip spheres. Sensitivity to potential differences of $0.1 \mathrm{mV}$ are typical for this type of measurement, with a dynamic range of about 100 dB. Both Automatic Gain Control (AGC) and fixed gain channels were used in the PWR in order to maintain resolution over the large dynamic range. Preamplifiers were protected against high charging potentials (above 88 v) by shorting neon lamps. Above about $1 \mathrm{MHz}$, the input signal is derived from Weitzmann metallic boom elements that support the spheres. DC electrical contact to the plasma is assured using a $10-\mathrm{cm}$ electrically conducting strip midway along each Weitzmann boom. Except for this conducting strip, the booms are insulated, thus forming a dipole antenna for high frequency waves.

In the initial part of the flight, the BEAR payload was oriented so that the beam fired along the magnetic field direction. The PWR booms were oriented perpendicular to this direction. As apogee was approached, the payload was tipped over so that the beam was fired perpendicular to the the magnetic field. In order to sample the distribution of plasma waves with respect to the magnetic field direction, the BEAR payload was then spun up to about $20 \mathrm{rpm}$ about its long axis. This allowed the PWR booms and sensors to rotate through the magnetic field direction. Spin modulation of the plasma wave spectrum then provides a direct signature of the direction of the wave vectors.

\section{IV.2 Instrument Development}

For the BEAR program we wanted to make measurements from DC into the low VHF range and to use available antennas and booms. A large dynamic range was required although extreme sensitivity was not paramount since only relatively large signal levels were of significance. Compactness, lightness of weight, low power consumption, and ruggedness were all design considerations. A block diagram of the PWR is shown in Figure IV.3. From this figure we see the three major components of the PWR: the DC/VLF receiver, the Baseband receiver, and the SFRs. Also indicated are the frequency bands for each and the signal sources (i.e., spheres or metal boom elements). 


\section{IV.2.1 Baseband and Preamplifiers}

Preamplifiers were installed in the boom-tip spheres in order to obtain a frequency response from DC to about $1 \mathrm{MHz}$. We used a Harris HA2-5162-5 operational amplifier and carefully compensated it for a gain of 1 while driving the load of the 3 conductor shielded cable used in the deployment mechanism for the booms. These amplifiers have an input resistance $>10^{11}$ ohms and input capacitance of about $3 \mathrm{pF}$. Because of the high input impedance the spheres can float with the local plasma potential and provide a voltmeter-like measurement of electric fields. It was necessary to use precision resistors with good RF characteristics for the gain elements to guarantee the results of the differential amplifier to follow. We used a pair of $4.7 \mathrm{kohm}$ resistors in series between the spherical probe and the input of the amplifier. From the midpoint of these resistors we connected a small neon lamp to ground in order to limit the input voltage to a safe value. We intended also to use zener diodes to further limit the input, but the diode capacity was not tolerable at the higher frequencies. The neon lamp limits at 88 volts which is above the published limit for the amplifier, but tests during which 115 volt $60 \mathrm{~Hz}$ power was applied through a simulated plasma resistance of 1 Megohm convinced us that the amplifier was adequately protected.

The preamplifiers are differenced with another Harris 5162 and the difference is distributed to two of the stepping receivers and to Baseband and DC/VLF electronics which process the waveform for telemetry in a variety of low frequency bands.

What we called the "Baseband" amplifier had a bandwidth from 10 $\mathrm{kHz}$ to $1 \mathrm{MHz}$ and modulated its own S-band telemetry transmitter. The design of the baseband amplifier was dominated by considerations of dynamic range and gain control. We wanted to be able to change gain as a function of frequency so that large signals in one part of the band would not cause gain reductions and preclude observation in another part of the band. After several false starts and some unbelievably complicated rough drafts we compromised on a two-passband AGC system built with LC filters using identical components. The high pass and low pass filters overlapped at a cutoff frequency of $100 \mathrm{kHz}$. The two bands were then amplified in separate AGC amplifiers and recombined into a common signal to be telemetered. With equal gains on the two channels the recombination, including phase shifts in the filters, reproduces the original input. Large signals in one band can be turned down without affecting the other. The AGC state is allowed to change only at discrete times and the actual state is telemetered over another channel. Figure IV.4 is a block diagram of the PWR showing the preamplifiers in the boom tip spheres, the Baseband receiver, the DC/VLF receiver (described below), the digital controls (described below), and the interface to telemetry. 


\section{IV.2.2 DC/VLF Receiver}

The DC/VLF receiver measures the electric field waveform with a maximum bandwidth of $10 \mathrm{kHz}$. The system is very similar to those successfully used by Cornell University for may years and was provided by Systems Unlimited of Ithaca, NY. As shown in Figure IV. 4 the instrument consisted of six data channels, each with different gains and band passes. The DC/VLF receiver measures the potential difference between the two boom-tip spheres. With high input impedances to the preamplifiers, very little current is drawn by the spheres and they assume the floating potential of the local plasma. Under these conditions, the electric field is obtained by dividing the potential difference between the spheres by the sensor separation. At low frequencies and long wavelengths the measurement is accurate to within $10 \%$, typically.

Three of the channels are DC-coupled and therefore able to measure electric fields with frequencies as low as the spin rate of the rocket $(0.3 \mathrm{~Hz})$. These "DC" channels each have different gains to allow for a large dynamic range. Since the telemetry rates are low it is easier to allow for multiple channels than to provide gain control.

AC-coupled measurements with a lower cutoff frequency of $10 \mathrm{~Hz}$ allow for filtering of the large amplitude spin-related signals. Therefore, much higher gain and sensitivity is possible. Two AC-coupled channels are utilized in the DC/VLF receiver; one with a fixed gain (10 $\mathrm{Hz}-2 \mathrm{kHz})$ and one with $\operatorname{AGC}(10 \mathrm{~Hz}-10 \mathrm{kHz})$.

One channel was dedicated to measuring the potential difference between sphere \#1 and the rocket body. The frequency response was DC to $500 \mathrm{~Hz}$ and the measurement limited to voltages below about $5 \mathrm{~V}$.

\section{IV.2.3 Stepped Frequency Receivers}

Because of telemetry limitations stepped frequency receivers (SFRs) were used for high frequency measurements. A design goal of 1 $\mu \mathrm{V}$ at the input to the receivers was set, a level typical of commercial radio equipment. We knew that RF noise generated in the rocket would be serious. To avoid interference we chose a rather narrow bandwidth ( 30 $\mathrm{kHz}$ ) which could be stepped through frequency-space avoiding known interference. A block diagram of the receivers is shown in Figure IV. 5 .

\section{$\underline{\text { RF Amplifier and Antenna Coupling }}$}

Preamplifiers in the boom tips ars useful to about $1 \mathrm{MHz}$ and thus, for lower frequencies it is possible to make a "voltmeter" measurement of electric fields in the plasma. The outputs of the 
preamps are differenced and coupled into two of the scanning receivers as well as the DC/VLF and Baseband receivers. For frequencies above 1 $\mathrm{MHz}$ we used the metallic arms of the Weitzmann booms as antennas. These booms are formed from coiled metal strips much li.e the wand of a tape measure. After deployment, each boom forms a $2.75-\mathrm{m}$ long tube with a 1 $\mathrm{cm}$ diameter at the base. Electrical connection between overlying strips of the boom is fair, but is not known to be reliabie at radio frequencies where the inductive effect of the coil may be significant. The entire deployment mechanism of the boom is insulated from the structure of the rocket by Delrin standoffs which were designed to reduce electrical capacitance to the payload. The coupling between various plasma waves and the boom antenna remains the largest unknown in the ultimate calibration of the receiver. Laboratory experiments indicate that the antenna is tuned in the 1 to $10 \mathrm{MHz}$ range.

Fortunately, much can be learned about the local plasma waves (spectral shape, growth and decay times, etc.) without an absolute calibration.

The two antennas are electrically connected through a six turn coil with a ferrite rod core material, similar to those used for broadcast band AM receivers. The center of the coil is grounded to the rocket frame to minimize effects of high charging voltages. The ferrite rod loosely couples energy into 8 secondaries tuned at different frequencies. The secondaries vary from 195 turns to 2 turns depending on the frequency range of the associated receivers. The lower frequency coils are placed at the ends of the ferrite rod and the primary (antenna) coil is at the center. In principal the secondaries accept energy only at their resonant frequency and this 8-way splitter does not have losses associated with directing RF energy to a receiver which cannot use it. Other than to check function, we made no sophisticated tests of the coupler or its efficiency.

Each secondary of the coupler is connected at high impedance to an FET amplifier stage which is in turn transformer coupled to a 50 ohm output. A DC voltage from each stepping receiver controls the capacitance or varactor diodes to provide tuning. The required voltage is determined experimentally and then generated from a lookup table by the microprocessor responsible for stepping the receivers. We provided for temperature compensation of this voltage to correct for thermal effects in the ferrite, but it was not necessary. The tuning of the secondaries is significant in reducing the sensitivity of a receiver to the emissions from the local oscillators of its neighbors.

\section{IF Amplifier}

Receiver selectivity and most of the receiver gain is concentrated in a two-stage intermediate frequency (IF) amplifier which runs at $12 \mathrm{MHz}$. With permission, we used a design originally developed for aeronautical communications radios by Narco Avionics of Fort Washington, PA. Bandwidth is determined by crystal filters using 12 pole quartz resonators with a bandwidth of about $30 \mathrm{KHz}$. Interstage coupling is accomplished by means of tuned transformers. This tuning is 
broad and does not contribute to the selectivity of the receiver. To control gain a Motorola MC1350 integrated IF amplifier was chosen because of its relatively low power consumption and ease of gain control.

We included diode detectors at the output of each of the two tuned stages to allow low gain data to be recovered in the event of very large signals. The low gain channel passes through only one of the two crystal filters and thus has less out-of-band rejection than the high gain channel.

The receivers use a dual gate field effect transistor (3N200) as a combined IF amplifier and mixer to drive the first crystal filter at the intermediate frequency. The FET requires very little power in the local oscillator and has proven adequate for keeping local oscillator energy out of the antenna circuit.

\section{Local Oscillators}

An easily controllable local oscillator was designed using a digital phase-locked loop. This design had the additional advantages of keeping local oscillator power to a minimum, reducing radiated energy and conserving battery power. It was also possible to choose frequencies for observation which would avoid known sources of interference. We used a Hartley oscillator with an unbuffered CMOS inverter as the active element and a dual varactor diode with a parallel coil for tuning. We were then able to use the other inverters on the chip as the buffer amplifier for the mixer, sine-to-square converter for the digital counter, and servo filter for the phase-locked loop. The oscillator fits in its own milled cavity separate from other electronics and runs from a single 5-volt power source. Feedback for stabilization is provided by a frequency synthesizer (Motorola MC145147). Using a computer program to simulate the loop, we were able to select filter components to optimize stabilization time at about 2 ms.

The tuning range of the local oscillator is limited by the capacitance range of the varactor diode and we were not able to get more than one octave without changing the inductance of the coil. For most cases this limitation sets the available stepping range of each receiver.

\section{Audio Amplifiers and AGC}

The detected outputs from the IF amplifiers are filtered and amplified by the "audio" stages, which are DC-coupled low-pass filters. They prepare the detected signal from each channel for conversion to digital form. Tracking analog-to-digital converters follow these signals to measure the maximum, minimum. and average amplitude at each frequency step. 
The "audio" stage also filters pulse-width modulated information created by the digital electronics to permit intelligent control of the IF and RF gain of the receiver. Decisions to increase or decrease gain are made at the end of the measurement interval of each step, so that even if a particular frequency step produces off-scale results there is no confusion due to gain changes and associated transient effects during the measurement interval. of $\mathrm{f}$-scale points are not lost entirely because the low gain output from the IF amplifier is also digitized. We were very careful not to program frequencies in the stepping cycle which would respond to known sources of interference and cause unwanted reduction of gain.

\section{IV.2.4 Digital Design Considerations}

The stepped frequency receivers were controlled digitally by a microcomputer based circuit located in a control box and by logic circuitry located on a digital board in each of the 10 SFR boxes. A significant step in reducing the size and complexity of the printedcircuit boards was made by the decision to use a new digital logic technology called the Logic Cell Array (LCA), developed by Xilinx Inc. The LCA is a CMOS digital integrated circuit that may be user-configured (programmed) to provide a large amount of custom digital circuitry. The device provided a high-density low-cost alternative to the standard SSI/MSI CMOS devices that were initially used in the design. The programmable nature of the devices also allowed for circuit changes without requiring any physical modifications to the circuit boards. Since the configuration of an LCA is stored in internal RAM, the device must be configured each time power is first applied, and may be reconfigured at any time. This feature was used in the design of the stepping receivers to allow the instrument to be reconfigured via an RS232 line from an external personal computer.

A block diagram of the control box electronics is shown in Figure IV.6. The control box interfaced with the rocket telemetry system and provided the receiver boxes with the required power, frequency selection, and timing signals. An RS-232 interface was provided to connect the instrument to a personal computer to provide a convenient way to test, modify, and verify the operation of the instrument. This interface was carried through the rocket pull-away umbilicals so that these functions could be utilized at various times during the period between integration and launch.

The $80 \mathrm{C} 31$ single-chip microcomputer from Intel Corp. was selected as the heart of the control box microcomputer circuit. The selection was based on its speed, low power, ease of use, and on-chip peripheral features. The microcomputer cilcuit included memory for program, instrument configuration data, and frequency selection storage. An LCA provided the interface circuitry nesded to generate and multiplex the receiver box control signals. 
The digital boards in the receiver boxes shown in Figure IV.5 directly controlled the frequency step, gain, and preamp tuning of the receiver. It provided 8 -bit analog-to-digital (A/D) conversions of the receiver's high and low gain output signals, and the circuitry to transfer the data to the telemetry system via the control box. All the custom digital logic was implemented on three LCAs.

The $A / D$ conversions in the receiver boxes were accomplished by implementing a tracking A/D converter algorithm. Two LCAs were used to implement 6 tracking $A / D$ converters to measure the average, maximum, and minimum values for the high and low gain outputs. Shift registers were also implemented on the LCAs for transferring the data to the control box.

The third LCA was used to provide automatic gain control (AGC), preamp tuning, and frequency selection circuits. Shift registers were implemented to transfer frequency selection and preamp tune data from the control box, and to transfer $A G C$ and frequency settings back to the control box. The AGC and preamp tune control outputs made use of pulsewidth modulation circuits for converting the digital values into analog control voltages. Frequency control of the receivers were accomplished by loading the phase-locked loop frequency synthesizers with the selected divider values in the frequency selection shift registers.

\section{3 Bench Test and Calibration}

The testing and calibration of the PWR scanning receivers was supported with personal. computer (PC) based simulators and calibration system. The simulators were implemented using a custom multifunction telemetry plug-in board for the PC which consisted of PC bus interface circuitry, a single-chip microcomputer, and a programmable logic cell array (LCA). The function of the board could be changed by down-loading different LCA configurations to the board from the PC.

The multifunction telemetry board was used to implement a telemetry simulation system for bench testing the scanning receivers. The simulator provided all the telemetry interface signals to the instrument, and the telemetry data was transferred to the PC. The telemetry data was shown on the PC in one of several graphic or text displays, and could be stored on disk for analysis and documentation purposes.

With a change in the LCA configuration, the simulator became the ground station display for the stepping receivers. The multifunction telemetry board served as the interface between the PC and the digital output of a Conic Data Systems' D/PAD Three unit. The D/PAD processed the serial PCM bit stream and provided the desired stepping receiver data in a parallel digital format. 
Testing and verification of the stepping receivers was also facilitated by the RS-232 monitor port on the receiver control box. A monitor program was run on the PC which could control the operation of the instrument via the port. Internal test routines in the control box could be selected as well.

The calibration of the stepping receivers was accomplished with an automatic calibration system, controlled by a PC, equipped with a multifunction telemetry board and an IEEE-488 interface board. The telemetry was simulated for the receivers while the program varied the amplitude and frequency of a signal generator, which was connected to the $R F$ input of the receivers. The results of the calibrations were processed by the PC and stored on disk. Figure IV.7 shows a typical calibration curve for one of the stepping receivers. The solid line plot is for the high gain measurement and the dotted line is for the low gain measurement. The curves are folded as the gain step changes; each new gain step is coded by a number or letter. Beyond about $10 \mathrm{mV}$ the receiver is in saturation.

The DC/VLF and Baseband receivers are both designed to measure electric field waveforms in the ionosphere. Because the measured data must be filtered (to prevent aliasing, modulating transmitters out of band and increase the signal-to-noise ratio) it is necessary to carefully measure the effect that these filters have on signals. Thus, the receivers were calibrated by determining gain and phase shift of each of the data channels.

In the case of the Baseband receiver, a precise signal generator and digital oscilloscope were used to perform the calibrations. The Baseband had AGC in two different frequency bands with seven discrete steps in each band. Thus calibrations were performed for each possible combination of gains. A typical calibration plot is shown in Figure IV.8. The plot is for gain state 0-2, that is, a gain state of 0 for the band $10 \mathrm{kHz}$ to $100 \mathrm{kHz}$ and a gain state of 2 for the band $100 \mathrm{kHz}$ to $1 \mathrm{MHz}$.

The DC/VLF receiver calibration was performed similarly to the Baseband but was automated using an computer controlled signal generator and $A / D$ system. The DC/VLF receiver is not a single data channel but instead a series of channels with different gains and pass bands. A calibration plot for one of the channels is shown in Figure IV.9.

IV.4 Environmental Tests

The instrument level environmental testing of tise PWR consisted of vibrational test (shake and shock) and thermal cycling. In all of these tests the PWR was 'powered on'. Sevcral data channels were monitored during the tests to confirm the hoalth of the instruments and all channels were checked after each test. Separate system environmental checks were also made at both AFGL and LANL. 
The specifications for the environmental testing were provided by LANL and are listed in the Appendix. The vibrational tests consisted of a random shake (spectrum: +6dB/octave for $20-40 \mathrm{~Hz}, 0.005 \mathrm{~g}^{2} / \mathrm{Hz}$ for 40-2000 $\mathrm{Hz}$, with composite amplitude of $3.1 \mathrm{~g}-\mathrm{rms}$ ) for $60 \mathrm{~s}$ and a $25-\mathrm{g}$ shock for $11 \mathrm{~ms}$. The tests were performed in all three axes. All PWR components worked normally during the vibrational testing. The circuit boards were examined under a microscope after testing for visual signs of damage and none were found.

Thermal tests consisted of temperature cycling starting at room temperature $+25 \mathrm{C}$, cooling to $-24 \mathrm{C}$, heating to $+65 \mathrm{C}$, and returning to $+25 \mathrm{C}$. Three cycles were used with varying times for each cycle and varying length holds at the temperature extremes. During thermal testing a problem with the baseband receiver was discovered. The low frequency 3-dB point varied with the temperature. After completing the thermal test an examination showed that two capacitors were the wrong type. Capacitors with low temperature coefficients were installed and the baseband receiver was put through one temperature cycle. All other components operated normally during thermal testing.

\section{IV.5 Integration}

Integration consisted of several steps:

1) The instruments were installed in the payload.

2) PCM, power and signal interfaces were checked.

3) GSE interfaces and operation were verified.

4) Instruments were checked for proper operation in various configurations.

5) Performance of the instruments were compared to laboratory calibrations.

6) Performance of the Baseband transmitter, receiver and recording systems were verified.

7) Noise baselines were recorded and interference tests with other instruments were preformed.

8) After any environmental testing (e.g., shake or shock tests) the instruments were tested for proper operation.

The integration of the PWR instrument into the BEAR payload was accomplished with only a few minor technical difficulties. The PCM bit clock was keeping CMOS circuits powered and thus not allowing the SFRs to reliably reset at power up. Also long cable lengths for digital signals were causing bit errors. To solve these problems level shifters and line terminations were added to the SFR control circuits on all telemetry digital signal lines.

Line losses in the $5 \mathrm{~V}$ supply to the SFR.s required the addition of extra $5 \mathrm{~V}$ and $5 \mathrm{~V}$ RETURN wires. 
During integration it became apparent that a system was needed for setting receiving and recording levels associated with the baseband instrument. We decided to add a ground controlled calibration signal for this purpose. This $600 \mathrm{kHz}$ sine wave was turned on shortly before launch, received and recorded. The system assured that normal outputs of the baseband system would not saturate receivers and recorders. It will also allow for a post flight calibration of the instrument.

FM receivers and analog recorders are an integral part of the baseband receiver and provide the limitation on the frequency response of the system. We had set a goal of $1 \mathrm{MHz}$ maximum frequency response. The response of the entire system was carefully checked and showed decrease in signal amplitude of about $3 \mathrm{~dB}$ from laboratory calibrations at $1 \mathrm{MHz}$. The loss in signal strength was less than expected and we have confidence that the system can make useful measurements up to 1 $\mathrm{MHz}$.

\section{Local Electrical Noise and Beam Interference Tests}

The PWR was operated in several test configurations to investigate whether EM noise generated by beam operation would affect the instrument. The PWh operated normally under all circumstances. Several data channels equipped with Automatic Gain Control (AGC) were capable of measuring the local electrical noise environment. Careful examination of the noise levels in various configurations (e.g., w/extractor, w/o extractor, w/motor adapter, w/bell jar, etc.) showed no beam related interference outside of acceptable levels.

Noise levels in the SFRs operating in the frequency range above about $3 \mathrm{MHz}$ limited the sensitivity of these measurements. The instrumental noise levels peaked near $12 \mathrm{MHz}$ (the IF frequency for the SFRs). The antenna was tuned near $3 \mathrm{MHz}$ (free space) thus coupling efficiency was low in the same frequency rarige where noise levels were high.

Noise generated by the ESA high voltage sweep were detectable on one channel $(10 \mathrm{~Hz}-10 \mathrm{kHz})$ with a level of about $100 \mathrm{uV}$ at the receiver. This could have affected the maximum sensitivity of the measurements, however, the beam-related signals during the flight where several hundred times this noise level.

In some configurations the Baseband instrument would reach its highest gain state and go into oscillation. This phenomena was never noticed in bench testing and may be related to the layout of the payload (i.e., boom, transmitter, line driver, cable locations). During the data taking period, as expected, the baseband was never in the highest gain state and thus this phenomena did not affect the performance of the instrument.

IV.6 Flight Performance and Data 
The Plasma Wave Receiver acquired electric field spectral information throughout the flight of BEAR when the boom antennas and tip spheres were deployed. All receiver boxes functioned as planned and no anomalies or instrument problems have been detected in the flight data thus far analyzed. Since this experiment was the largest user of telemetry PCM data, approaching the $800 \mathrm{kbps}$ maximum, and also used a dedicated analog telemetry link, the amount of data available for analysis is very large. Consequently, the analyses efforts envisioned for this experiment will last for some months to come. Below, we discuss some of the initial results based primarily on several channels of the low frequency waveform data.

Figure IV.10 shows a 15 -second interval of the $10-2000 \mathrm{~Hz}$ electric field data obtained while the BEAR payload was aligned with the magnetic field and the neutral beam was emitted parallel to the magnetic field. The electric field waveform measurements have been converted into a dynamic spectrum by Fast Fourier Transform techniques. The power spectral density is coded by the grey scale. In the panel below the dynamic spectrum, the Beam Current Monitor (BCM) of the Los Alamos National Laboratory diagnostics has been plotted. The net charged current for each beam pulse is plotted as a vertical bar. The next panel below the BCM is the Langmuir Probe (LP) measurements of the Naval Research Laboratory. This panel plots the probe currents for two probes, one held at a fixed bias and the other with a two cycles of a sawtooth bias, applied with a $50 \%$ duty cycle. The bottom panel is the sawtooth sweep monitor of the Langmuir probe. By examining the BCM data, we can see that the beam current pulses did not occur every $200 \mathrm{~ms}$ as planned; however, most of the beam pulses that did occur are near the -1 mA current that was planned. Associated with each beam pulse, we can see that a broadband burst of electrostatic noise is detected by the PWR. The relationship is such that the beam pulse is aligned with the leading edge of the PWR noise burst. Each electrostatic noise burst is about $0.25 \mathrm{sec}$ wide at the lowest frequencies; the 50 -usec width of the beam pulse cannot be resolved in the time scale of the plot. Thus, electric field fluctuations in the plasma surrounding the BEAR payload last about 5000 times longer than the beam pulse.

In Figure IV.11, we show the PWR data in the same format as the previous figure for the time interval when the beam neutralizer was turned off. The beam neutralizer-off period is from about 260-269 $\mathrm{sec}$ of the BEAR flight, and corresponds to the times when the BCM is at about $-10 \mathrm{~mA}$. At this time in the flight, the BEAR payload was spinning at $20 \mathrm{rpm}$ and oriented so that the beam pulses were emitted perpendicular to the magnetic field. The PWR spectrum shows intense broadband noise for this period associated with every beam pulse. The two data figures shown also indicate the effects of the gas thrusters used for at titude control. Low frequency lines in the PWR spectrum, from about $10 \mathrm{~Hz}$ to $200 \mathrm{~Hz}$ appear to be associated with thruster firings. These lines may be acoustic modes stimulated in the plasma by the thrusters, however, these effects have not been analyzed. The LP measurements in both figures also show lepletions of the local plasma density associated with each thruster firing. Both the PWR and LP 
measurements need further analys is in order to determine how such density depletions affect the vehicle charging when the beam is pulsed.

The investigation of charging of the BEAR vehicle was one of the main scientific objectives of the diagnostics measurements. The PWR included one $\mathrm{DC}$-coupled $500-\mathrm{Hz}$ channel which provided measurements of the potential of one of the tip spheres with respect to the vehicle. The measurements from this channel around the period of beam neutralizer-off are shown in Figure IV.12. Two outstanding features may be noted in these measurements: the polarity of the voltage pulses reverses during the neutralizer-off period, and the electric field associated with each potential pulse is primarily aligned with the magnetic field direction. The latter effect is shown by the signal from the roll magnetometer sensing the payload $y$-direction, shown in the bottom panel. The PWR booms were aligned with the payload y-direction, and when the detected electric field occurs at the extreme positive or negative excursion of the magnetic field we know that the electric field is aligned with the magnetic field direction. Both before and after the neutralizer-off period, the electric field directions of the vehicle charging shows no clear relationship to the magnetic field direction. Again, because the $500-\mathrm{Hz}$ channel cannot respond to the 50 usec time scale of the beam pulse, the potential measurements in Figure IV.12 relate to late-time vehicle charging after the initial charging transient is over. LP measurements, discussed elsewhere in this report, suggest that the initial charging transient (i.e., during the first 50 usec) produces both positive and negative potential swings.

The PWR Baseband receiver has a $1 \mathrm{MHz}$ response and was intended to detect electron plasma oscillations resulting from the impulsive charging of the BEAR vehicle during beam pulses. The Baseband measurements are differential measurements between two tip spheres and thus do not provide vehicle potential measurements. However, this waveform measurement is related to the time variation of the vehicle potential. An example of these measurements during a beam pulse is shown in Figure IV.13. The nearly coherent oscillations are clearly associated with the beam pulse and appear to represent a ringing of the plasma stimulated by the impulsive charging of the vehicle. Various theoretical studies suggest that these waves are electron plasma oscillations. A direct measurement from the plot indicates a plasma frequency of $200-250 \mathrm{kHz}$, corresponding to an electron density of $500-$ $800 \mathrm{~cm}^{-3}$. As seen in the figure, the frequency of the oscillations increases slightly after the pulse if over, and then decays with a $e^{-1}$ decay time of about $30 \mathrm{usec}$, or about 6 plasma periods.

The data discussed above show that the flight performance of the PWR experiment exceeded expectations and has provided a large data base with much information about the operation of neutral beam accelerators in space. We are planning to analyze this data and continue reports on the results for some time to come. 
Ionospheric Characteristic Plasma Frequencies

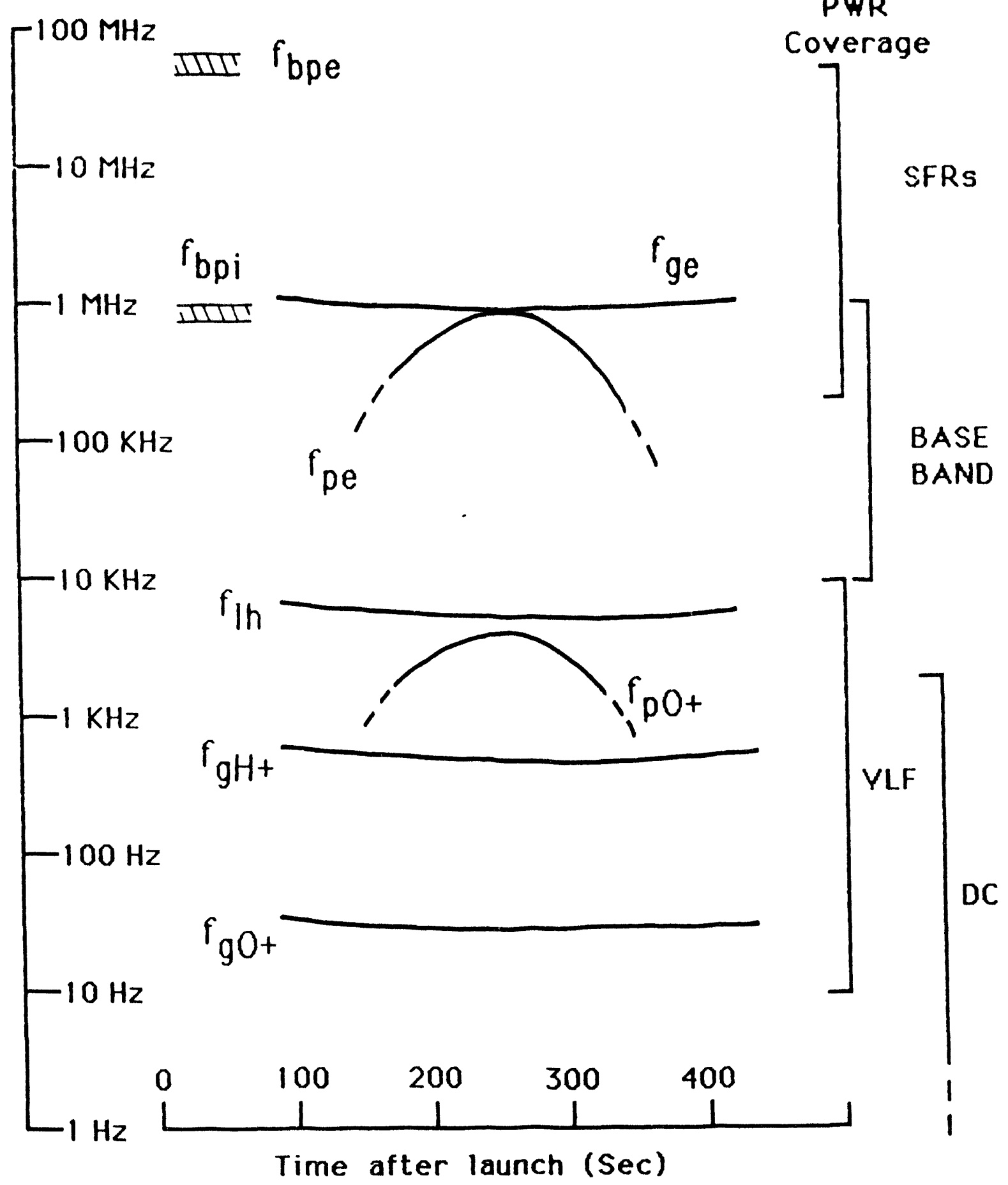


BEAR PWR Electric Field Spectrum Analyzer

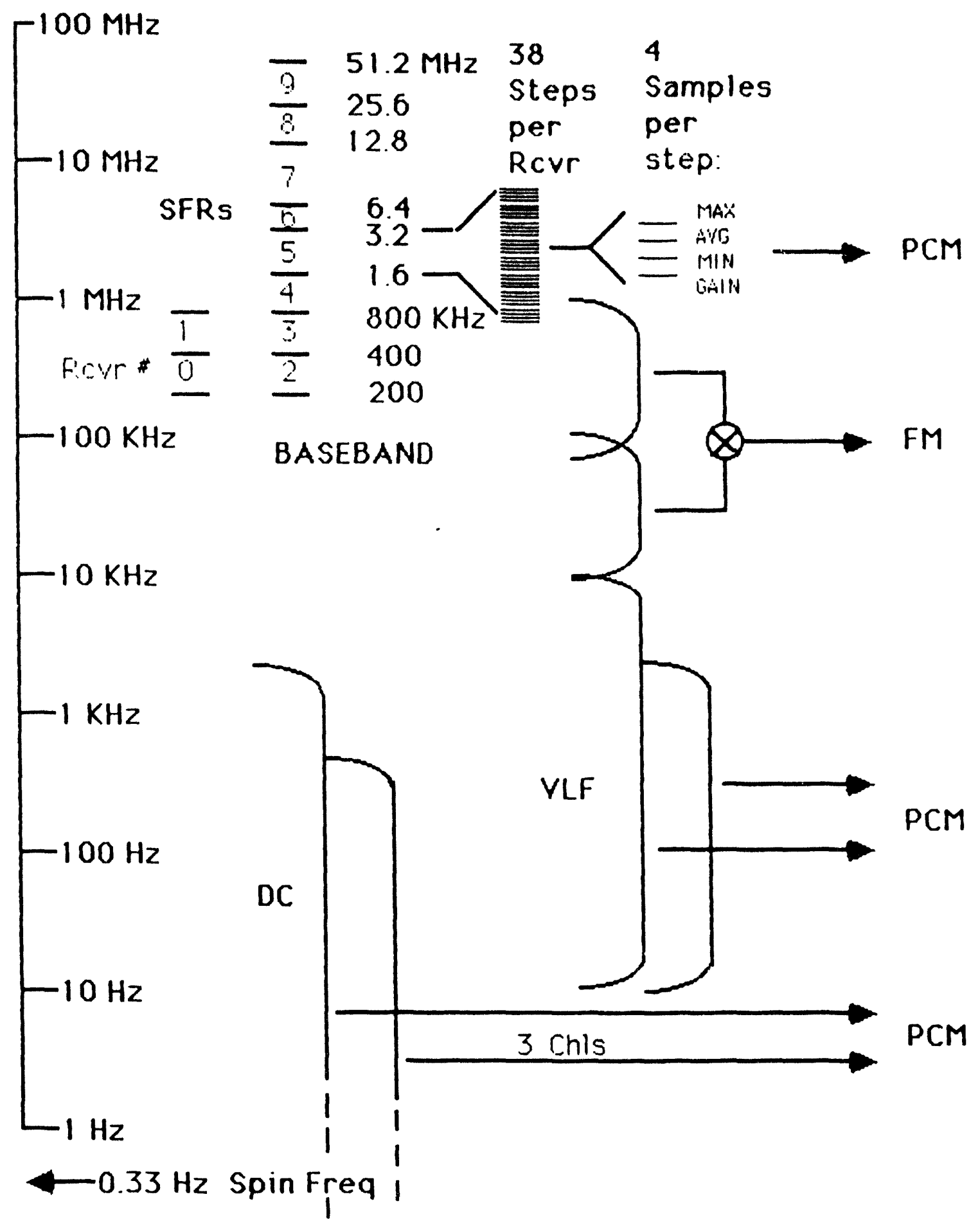

Figure IV.2 


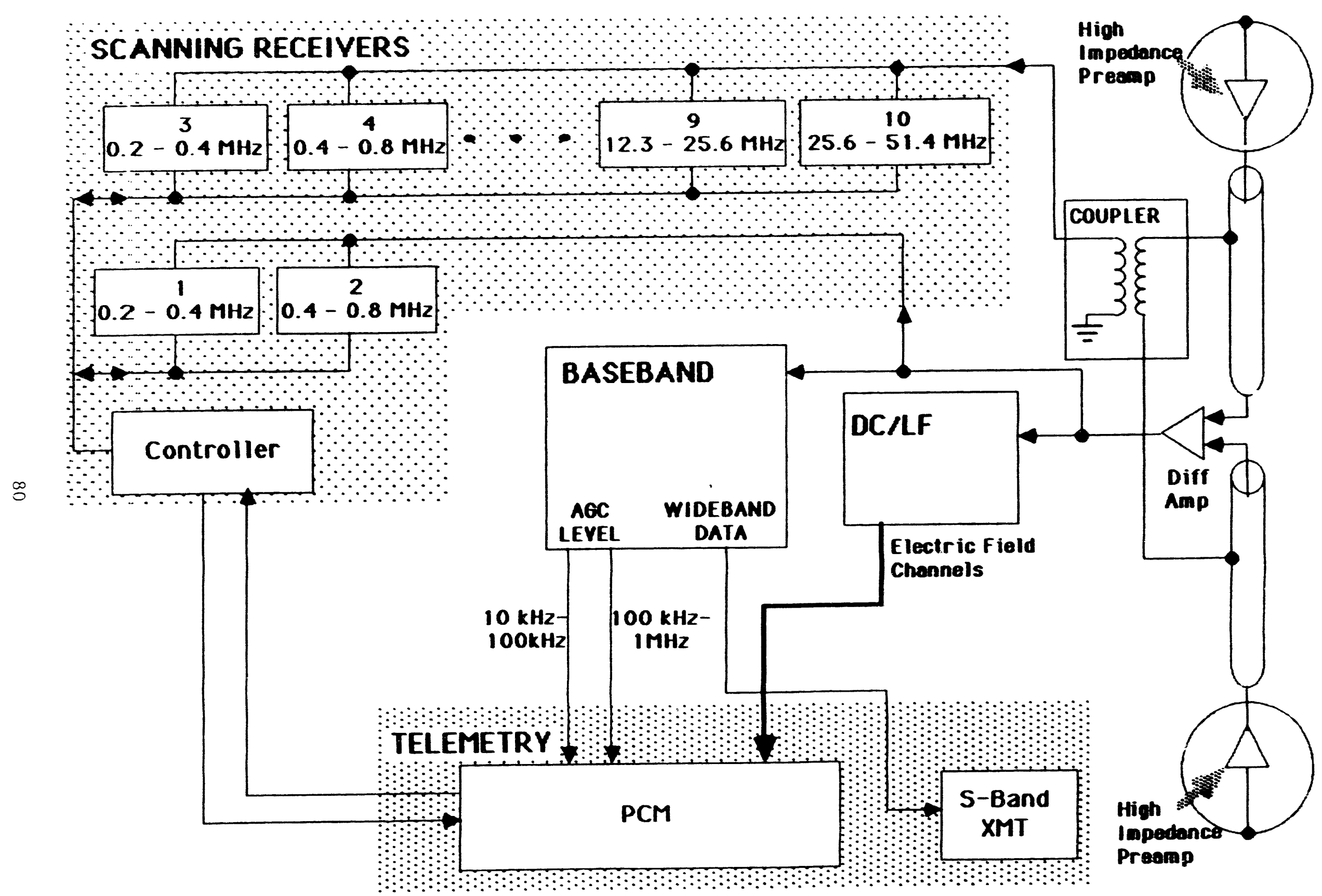




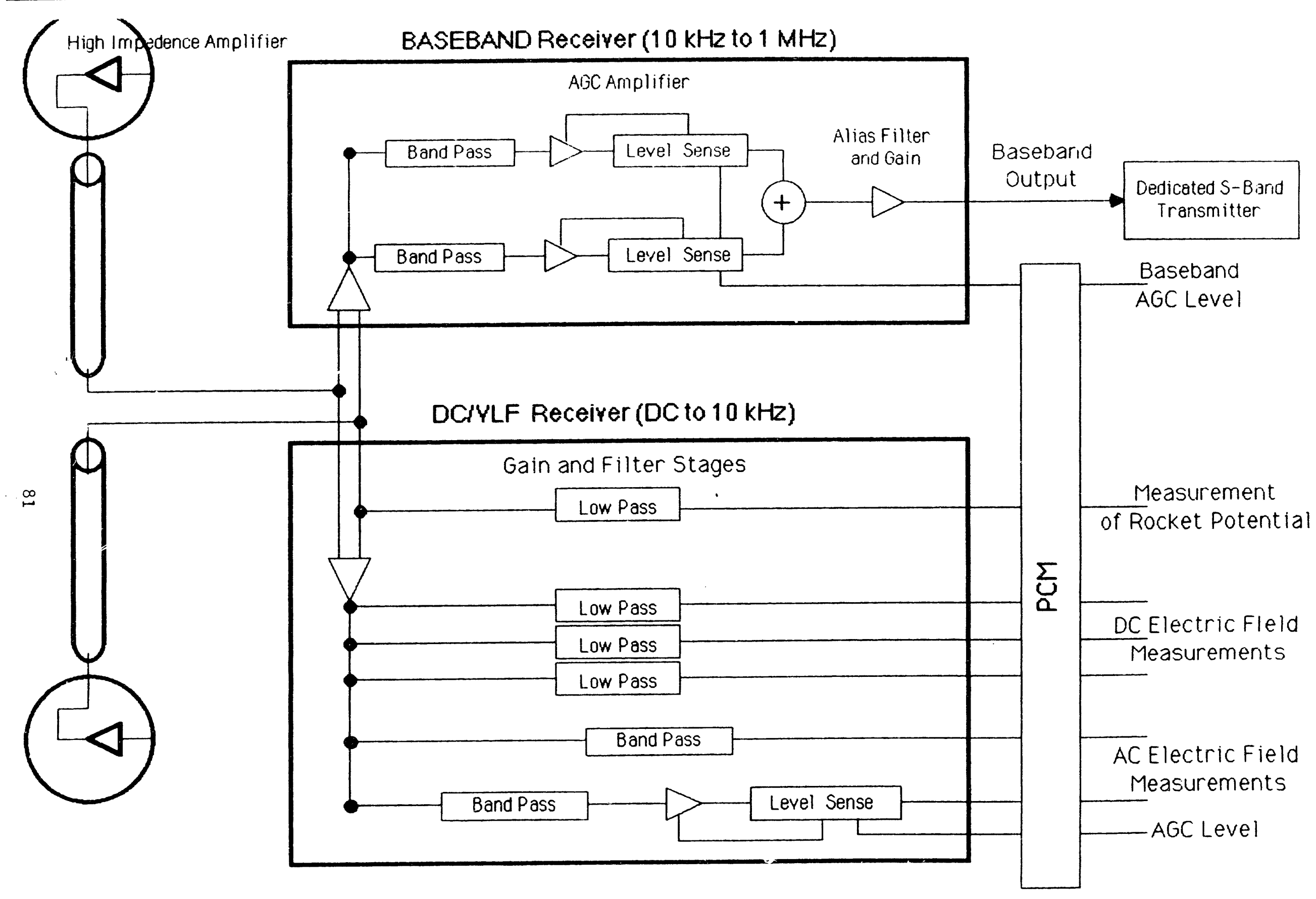

NRL Baseband and DC IVLF Receivers

Figure IV.4 


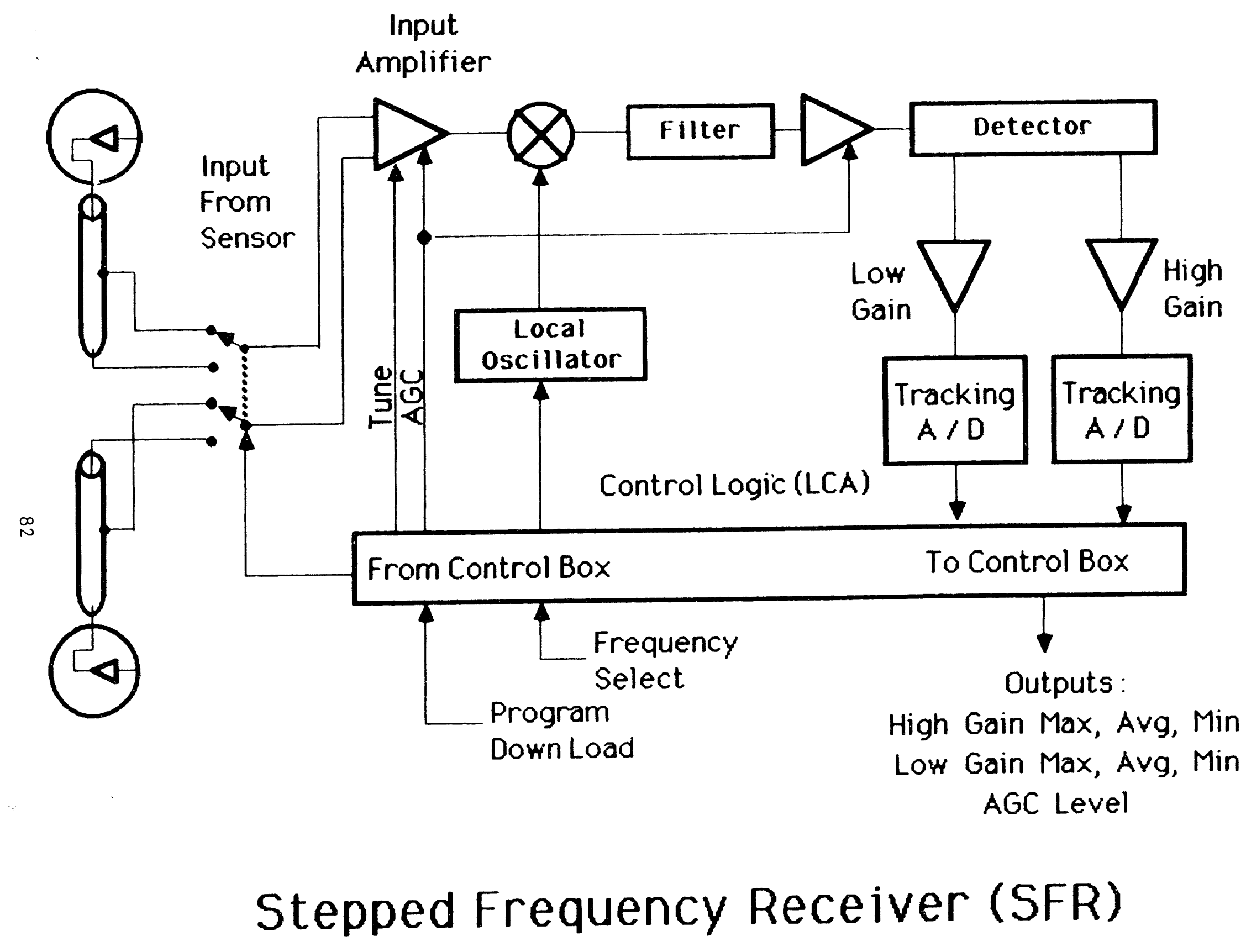

Figure IV.5 


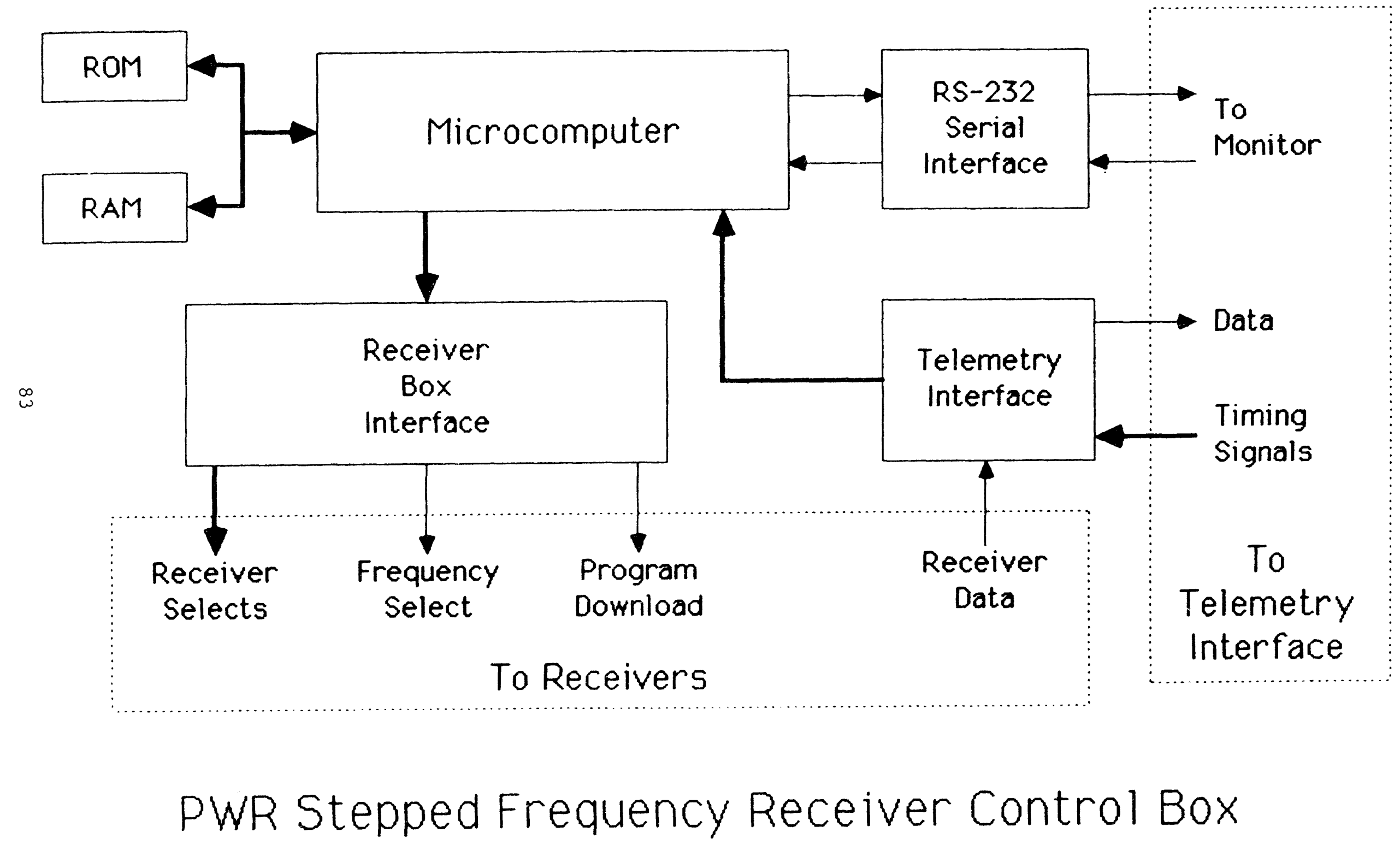




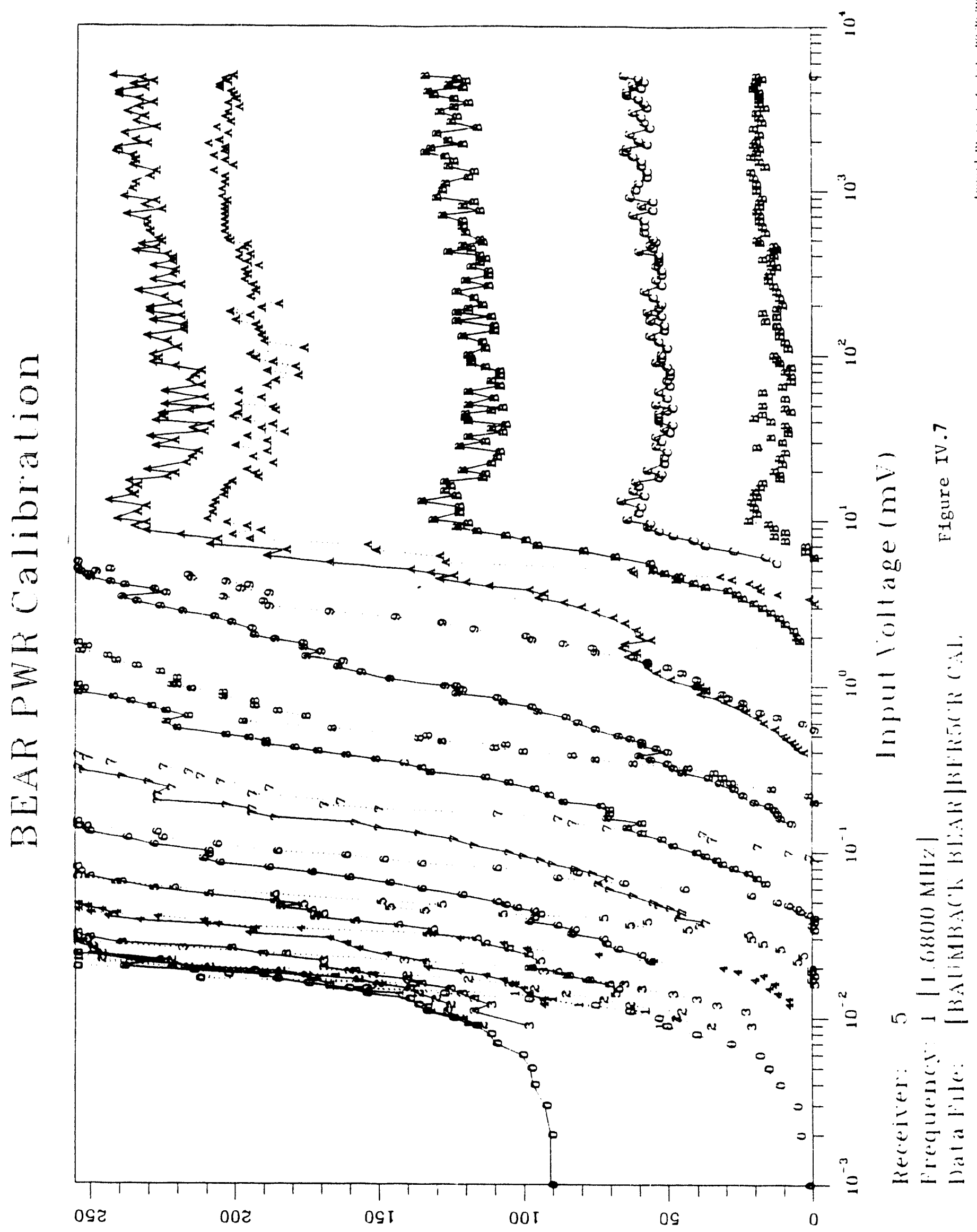

sfunoj zndzno 


\section{BEAR Baseband Calibrations}
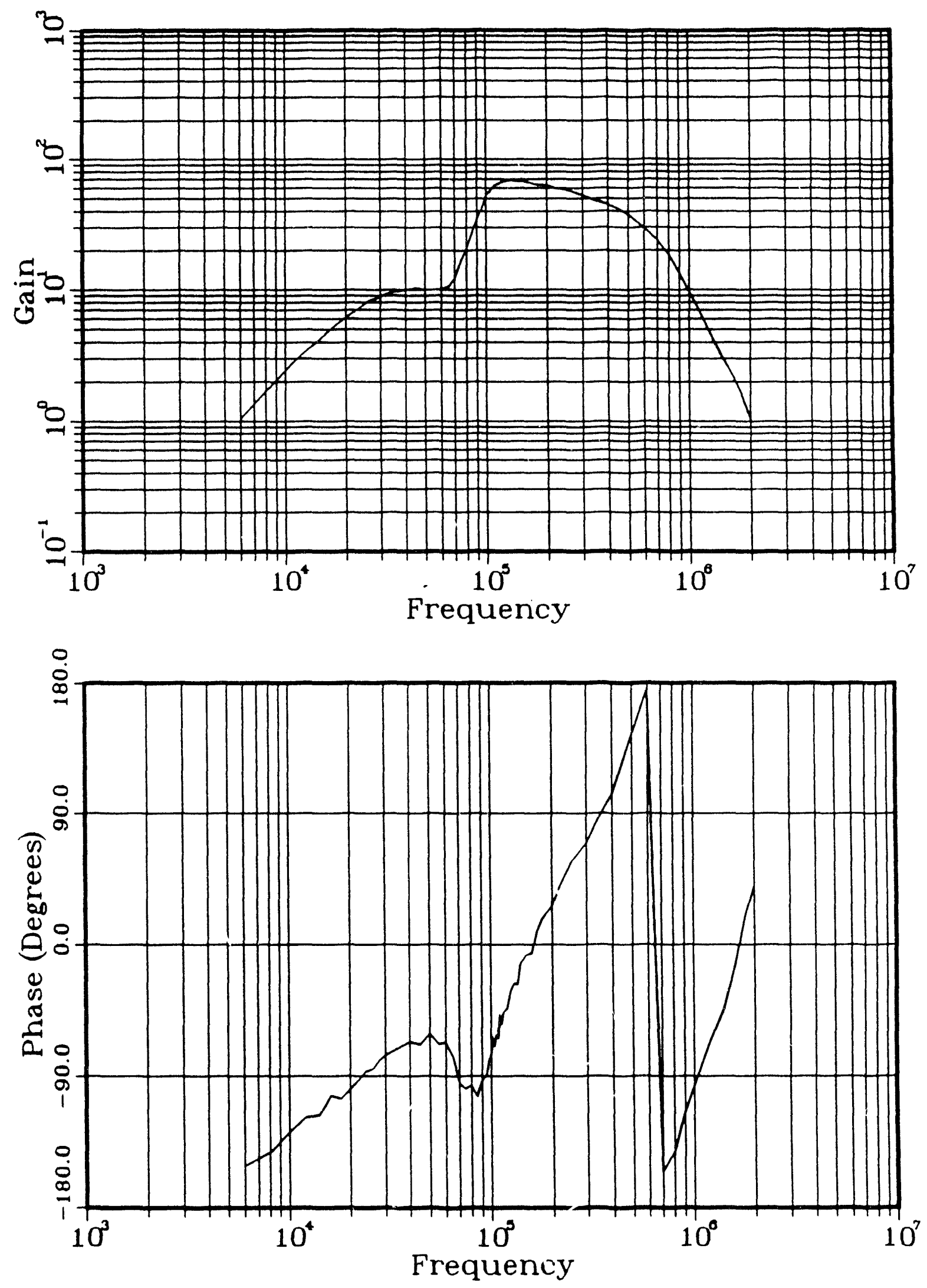

Eigure IV. 8 
NRL BEAR

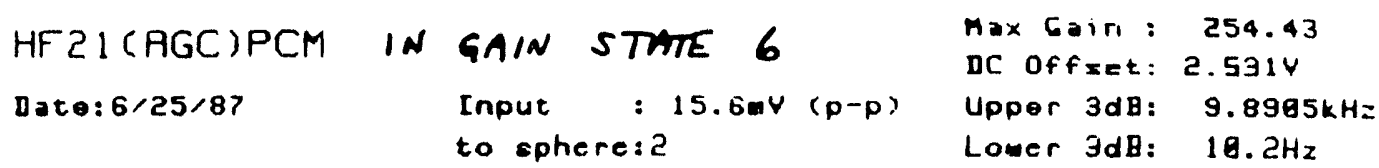

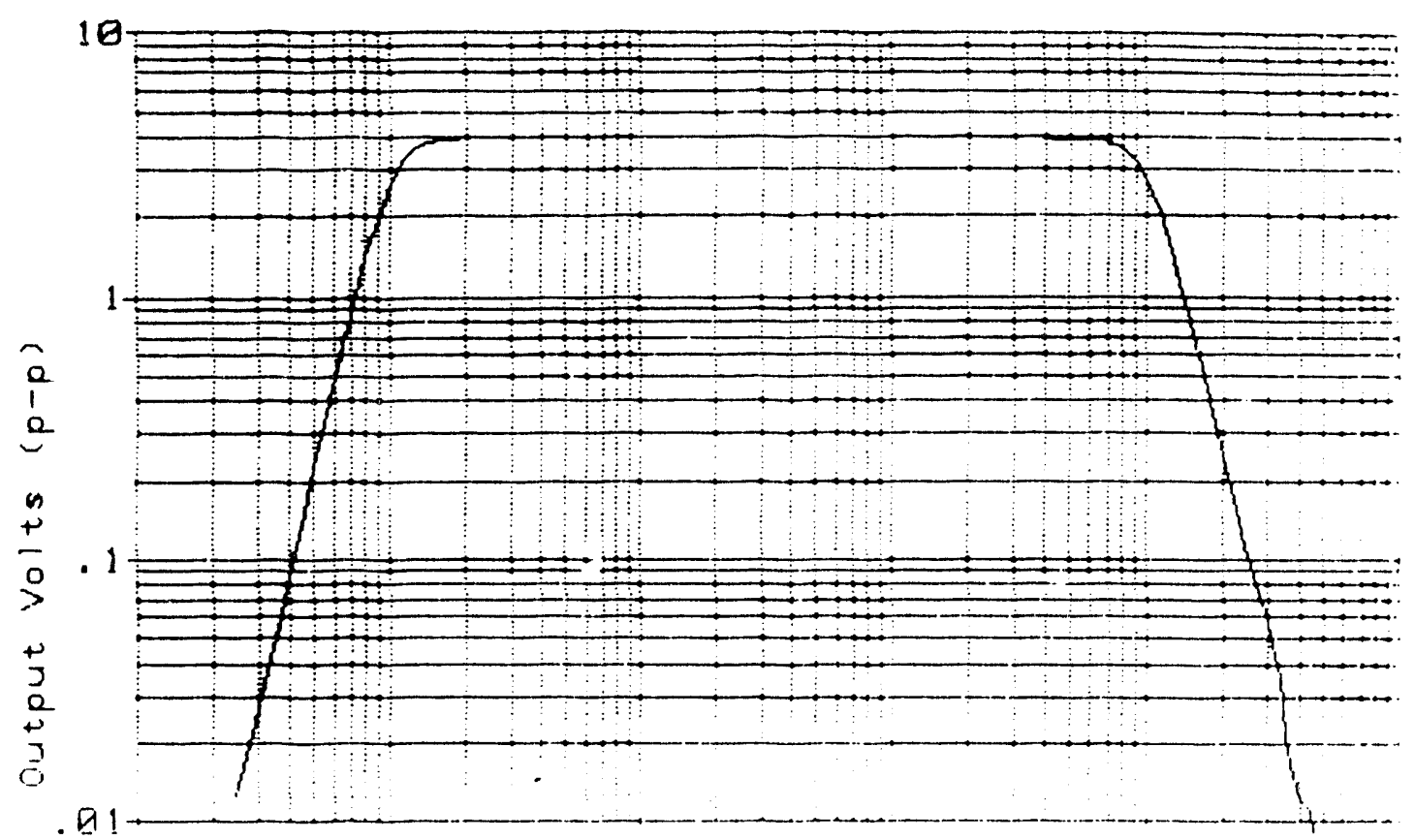

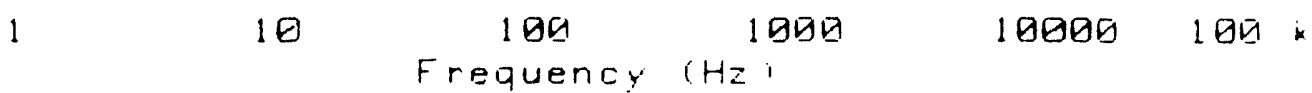

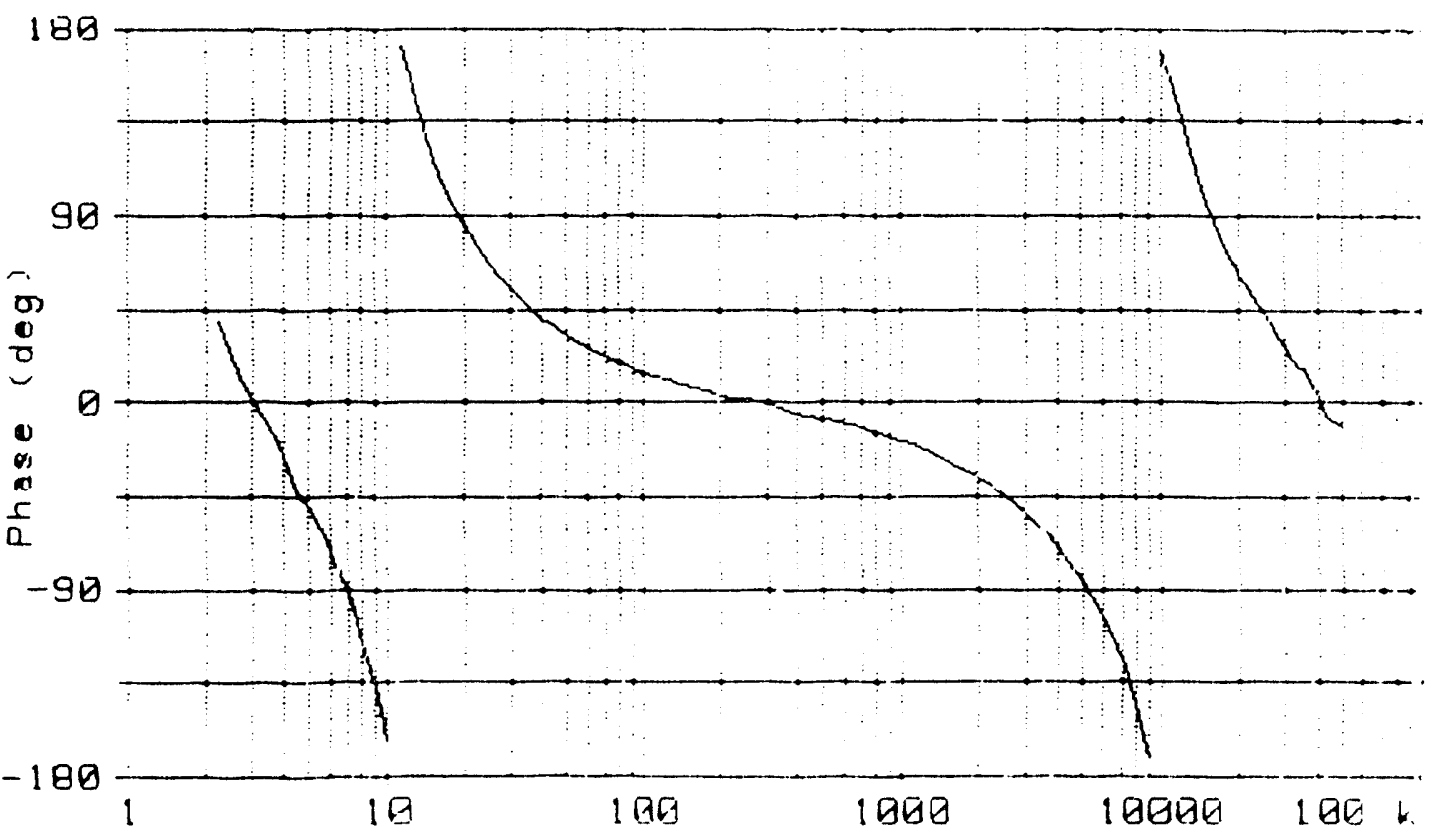

Figuce IV.9 

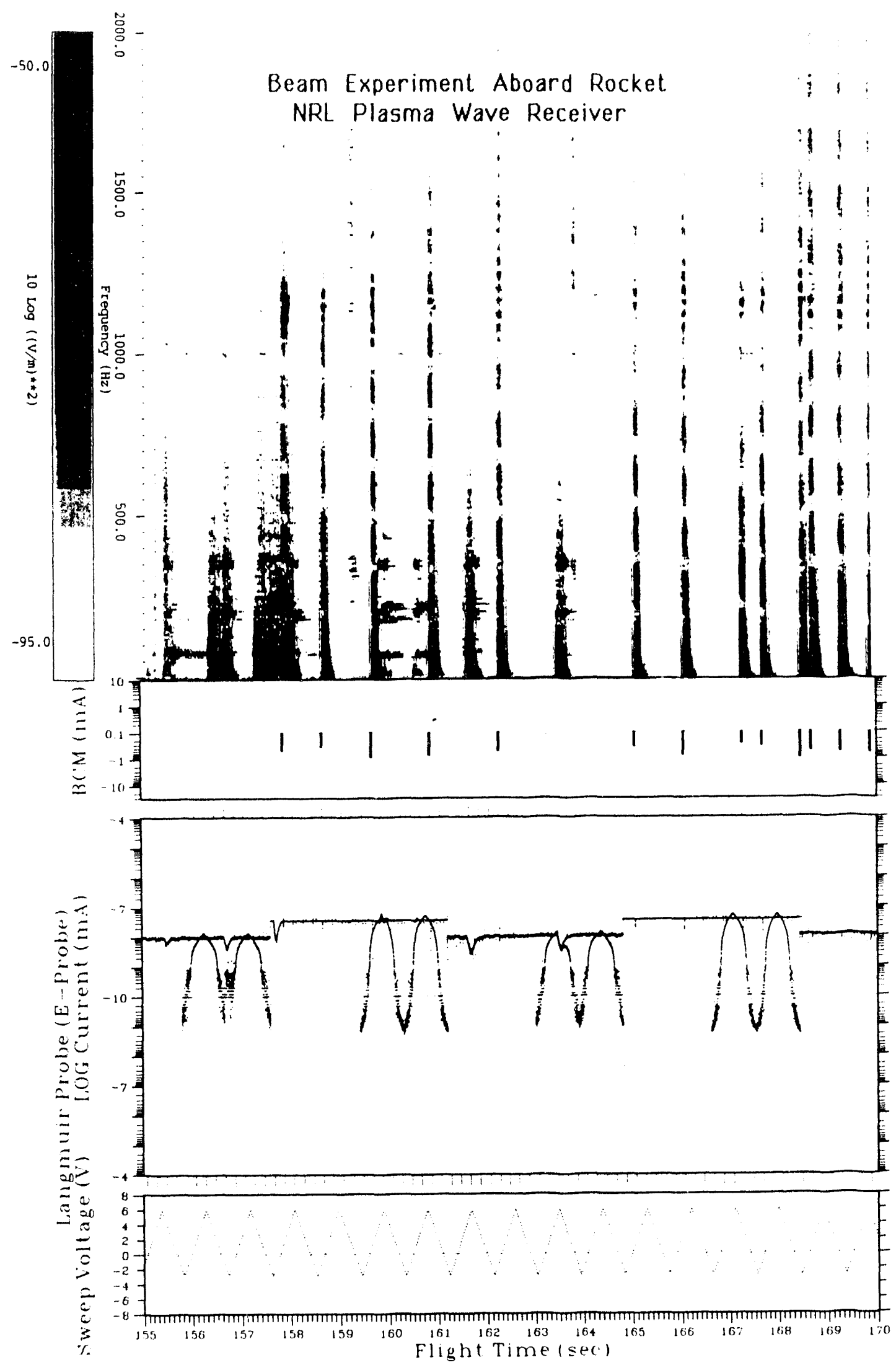

Figure IV.10 


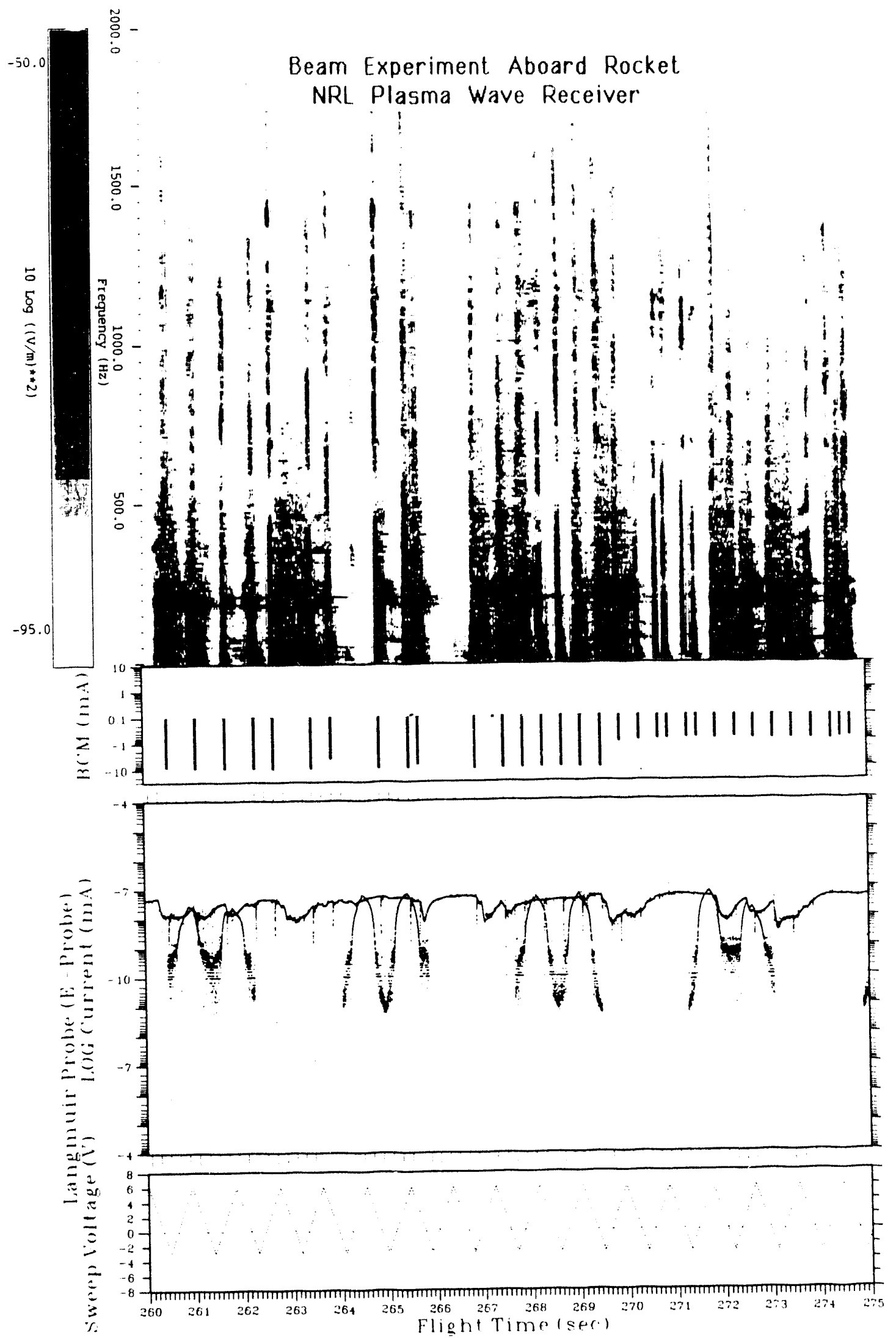

Figure IV.11 


\section{Beam Experiment Aboard Rocket}

NRL Plasma Wave Receiver

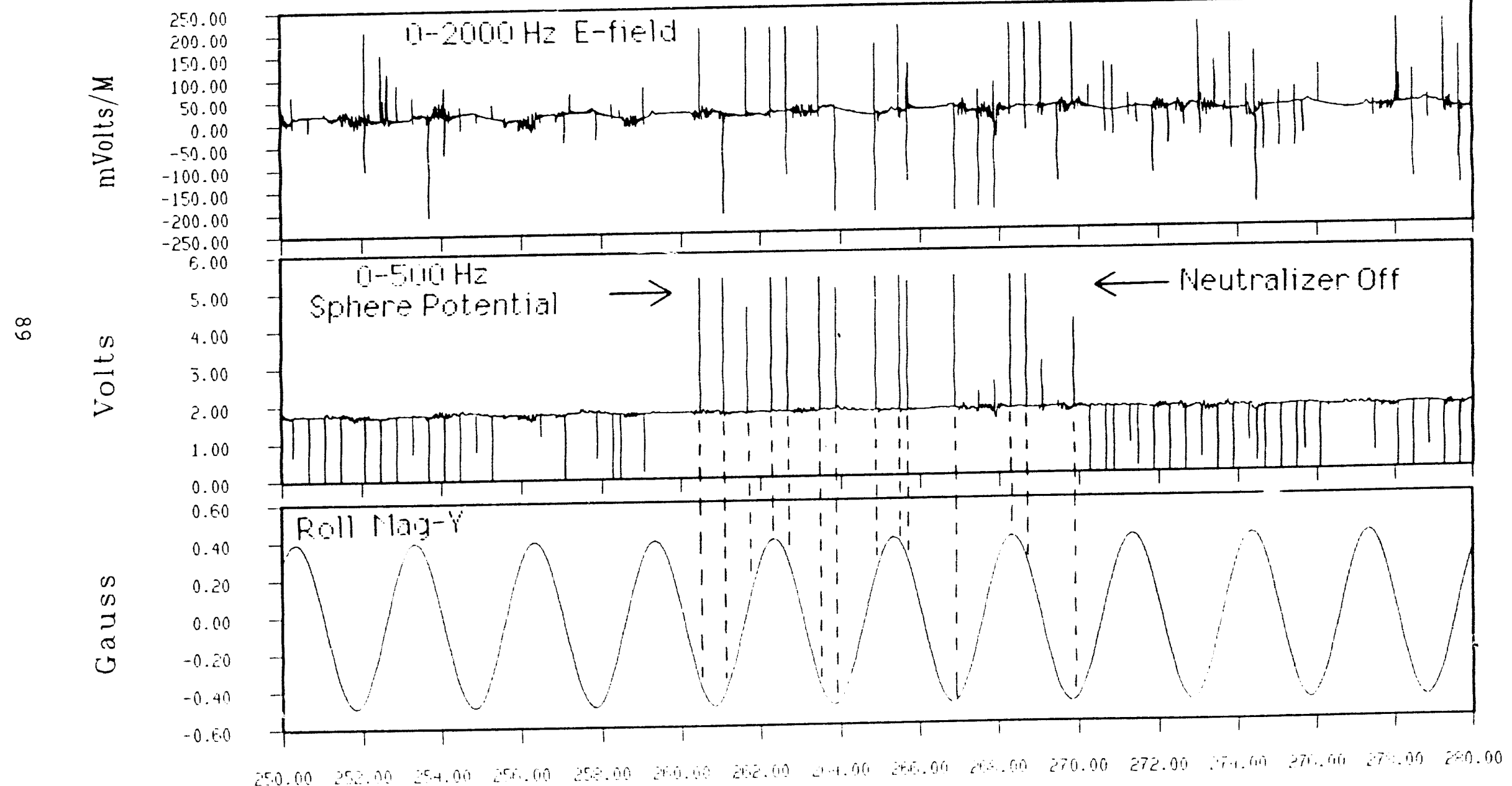

Time(s)

F1gure IV.12 


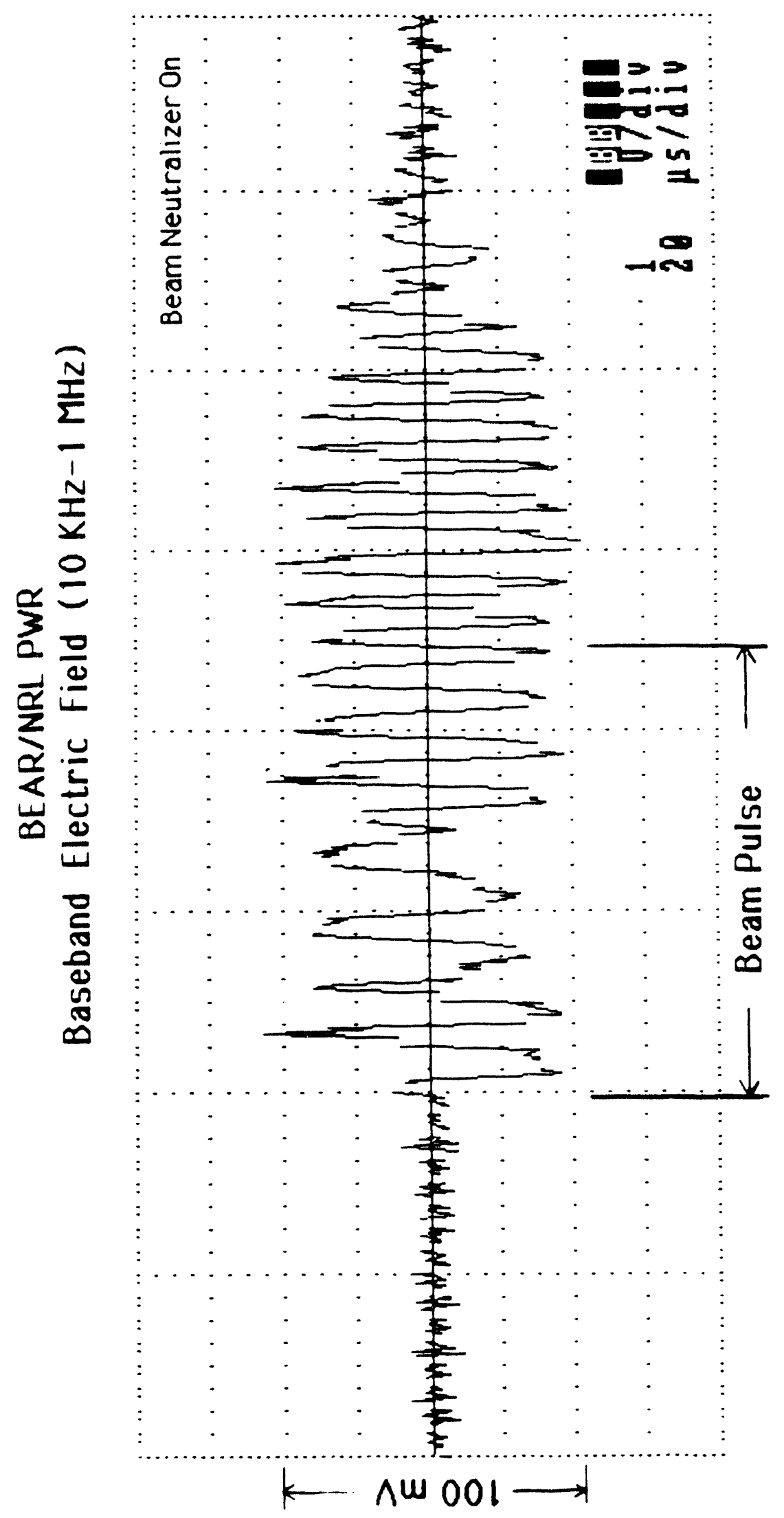

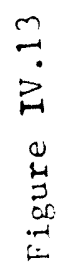


V. Appendix 


\section{Langmuir Hrobe Lalibration BEAR}

\begin{tabular}{|c|c|c|c|c|c|c|c|}
\hline AMP & $\mathbf{R}$ & MON & NPTS & SLOPE & $\operatorname{SIGA}$ & INT & $S I G B$ \\
\hline $01000 E+03$ & $0.5074 E+03$ & $0.1000 E-01$ & $0.5610 E+03$ & $0.2143 E-08$ & $0.9245 E-11$ & $-0.9527 E-09$ & $0.1382 E-10$ \\
\hline$-0.1000 E+03$ & $0.5074 \varepsilon+03$ & $0.2340 E+01$ & $0.4310 E+03$ & $-0.2114 E-08$ & $0.1280 E-10$ & $0.8635 E-09$ & $0.1756 E-10$ \\
\hline $0.3100 E+02$ & $0.1038 E+03$ & $0.1900 \mathrm{E}+00$ & $0.3480 E+03$ & $0.7002 E-08$ & $0.13495-10$ & $0.3609 E-08$ & $0.2091 \mathrm{E}-10$ \\
\hline$-0.3100 E+02$ & $0.1038 E+03$ & $0.2560 E+01$ & $0.3090 E+03$ & $-0.6968 E-08$ & $0.1826 E-10$ & $-0.3733 E-08$ & $0.2745 E-10$ \\
\hline $0.1000 E+02$ & $0.2984 E+02$ & $0.1200 E+00$ & $0.3150 E+03$ & $0.2077 E-07$ & $0.2328 \mathrm{E}-10$ & $0.2415 E-07$ & $0.3534 E-10$ \\
\hline$-0.1000 E+02$ & $0.2984 E+02$ & $0.27905+01$ & $0.2870 E+03$ & $-0.2076 E-07$ & $0.2694 E-10$ & $-0.2427 E-07$ & $0.10118-10$ \\
\hline $0.3000 E+01$ & $0.9960 E+01$ & $0.6400 E+00$ & $0.3500 E+03$ & $0.6904 E-07$ & $0.5478 E-10$ & $0.8051 \mathrm{E}-07$ & $0.8306 \mathrm{E}-10$ \\
\hline$-0.3000 E+01$ & $0.9960 E+01$ & $0.3010 E+01$ & $0.2720 E+03$ & $-0.6912 E-07$ & $0.7942 E-10$ & $-0.8009 \varepsilon-07$ & $0.1115 E-09$ \\
\hline $0.1000 E+01$ & $0.3010 E+01$ & $0.8600 \Sigma+00$ & $0.27708+03$ & $0.2090 E-06$ & $0.2008 \Sigma-09$ & $0.2765 \varepsilon-06$ & $0.2909 E-09$ \\
\hline$-0.1000 E+01$ & $0.3010 E+01$ & $0.3230 E+01$ & $0.2730 E+03$ & $-0.2088 E-06$ & $0.2458 E-09$ & $-0.2761 E-06$ & $0.3565 E-09$ \\
\hline $\mathbf{I}$ & $G(I)$ & \multicolumn{2}{|c|}{$R(O(I+1) / G(I))$} & $R(-G(I+1) /-G(I))$ & \multicolumn{2}{|c|}{ VSWI0/G(I) } & $v 00 / G(I)$ \\
\hline 1 & 100 & \multicolumn{2}{|c|}{$0.3268 E+01$} & $0.3296 E+01$ & \multicolumn{2}{|c|}{$-0.22 \leqslant 1 E-01$} & $0.4267 E+00$ \\
\hline 2 & 31. & \multicolumn{2}{|c|}{$0.2966 E+01$} & $0.2980 E+01$ & \multicolumn{2}{|c|}{$-0.6121 E-02$} & $-0.5255 E+00$ \\
\hline 3 & 10. & \multicolumn{2}{|c|}{$0.3324 E+01$} & $0.3329 E+01$ & \multicolumn{2}{|c|}{$-0.1766 E-02$} & $-0.1166 E+01$ \\
\hline 4 & 3. & \multicolumn{2}{|c|}{$0.3027 E+01$} & $0.3020 E+01$ & \multicolumn{2}{|c|}{$0.2101 \varepsilon-02$} & $-0.1162 E+01$ \\
\hline 5 & 1. & \multicolumn{2}{|c|}{$0.0000 \varepsilon+00$} & $0.0000 E+00$ & \multicolumn{2}{|c|}{$0.6348 E-03$} & $-0.1323 E+01$ \\
\hline
\end{tabular}

\section{(sensitive electrometer)}

TABLE $\mid 1.1$ 


\section{Langmuir Probe Calibration BEAR}

\begin{tabular}{|c|c|c|c|c|c|c|c|c|}
\hline AMP & $\mathbf{R}$ & MON & NPTS & SLOPE & SIGA & INT & SIGB & CORA \\
\hline $0.1000 E+03$ & $0.1190 E+01$ & $0.1: 20 E+01$ & $0.2120 E+03$ & $0.6524 E-06$ & $0.1107 \mathrm{E}-08$ & $-0.2759 \varepsilon-06$ & $0.1942 \mathrm{E}-08$ & C. $9997 E+00$ \\
\hline$-0.1000 E+03$ & $0.1190 E+01$ & $0.3500 E+01$ & $0.2050 E+03$ & $-0.6521 E-06$ & $0.1052 E-08$ & $0.2747 E-06$ & $0.1834 E-08$ & $-0.9997 \mathrm{E}+00$ \\
\hline $0.3100 \varepsilon+02$ & $0.3166 \varepsilon+00$ & $0.1350 E+01$ & $0.3020 \varepsilon+03$ & $0.2039 E-05$ & $0.1801 E-08$ & $0.1068 \mathrm{E}-05$ & $0.2776 \mathrm{E}-08$ & $0.9999 E+00$ \\
\hline$-0.3100 E+02$ & $0.3166 \varepsilon+00$ & $0.3720 E+01$ & $0.29208+03$ & $-0.2039 E-05$ & $0.1976 E-08$ & $-0.1066 E-05$ & $0.3026 E-08$ & $-0.9999 E+00$ \\
\hline $0.1000 E+02$ & $0.99548-01$ & $0.15708+01$ & $0.3180 E+03$ & $0.6437 E-05$ & $0.5507 E-08$ & $0.7474 \varepsilon-05$ & $0.8371 E-08$ & $0.9999 E+00$ \\
\hline$-0.1000 E+02$ & $0.9954 \mathrm{E}-01$ & $0.3940 E+01$ & $0.2040 E+03$ & $-0.6433 E-05$ & $0.7285 E-08$ & $-0.7422 E-05$ & $0.1065 E-07$ & $-0.9998 \varepsilon+00$ \\
\hline $0.3000 E+01$ & $0.3012 E-01$ & $0.1790 \mathrm{E}+01$ & $0.3260 E+03$ & $0.2175 E-04$ & $0.1877 E-07$ & $0.2524 E-04$ & $0.2859 E-07$ & $0.9999 \varepsilon+0 \mathrm{~J}$ \\
\hline$-0.3000 E+01$ & $0.30125-01$ & $0.1160 \mathrm{E}+01$ & $0.2860 z+03$ & $-0.2171 E-04$ & $0.2410 E-07$ & $-0.2535 E-04$ & $0.3453 E-07$ & $-0.9998 E+00$ \\
\hline $0.1000 E+01$ & $0.1001 E-01$ & $0.2020 \varepsilon+01$ & $0.3580 E+03$ & $0.6924 E-04$ & $0.1768 E-07$ & $0.9155 E-04$ & $0.7188 E-07$ & $0.9999 E+00$ \\
\hline$-0.1000 E+01$ & $0.1001 \mathrm{E}-01$ & $0.43908+01$ & $0.2600 E+03$ & $-0.6916 E-04$ & $0.9526 \mathrm{E}-07$ & $-0.9166 E-04$ & $0.1296 \varepsilon-06$ & $-0.9998 E+00$ \\
\hline
\end{tabular}

$\begin{array}{ccc}I & G(I) & (O(I+1) / G(I)) \\ 1 & 100 & 0.3126 E+01 \\ 2 & 31 & 0.3156 E+01 \\ 3 & 10 & 0.3380 E+01 \\ 1 & 3 . & 0.3183 E+01 \\ 5 & 1 . & 0.0000 E+00\end{array}$

$R(-G(I+1) /-G(I))$
$0.3127 E+01$
$0.3155 E+01$
$0.3375 E+01$
$0.3186 E+01$
$0.0000 E+00$

VSWI0/G(I)
$-0.6991 E-03$
$0.3027 E-03$
$0.2607 E-02$
$-0.1613 E-02$
$-0.5359 E-03$

$000 / G(I)$

$0.1221 \varepsilon+00$

$-0.52345+00$

$-0.1157 E+01$

$-0.1164 E+01$

$-0.1324 E+01$

\section{(insensitive electrometer)}




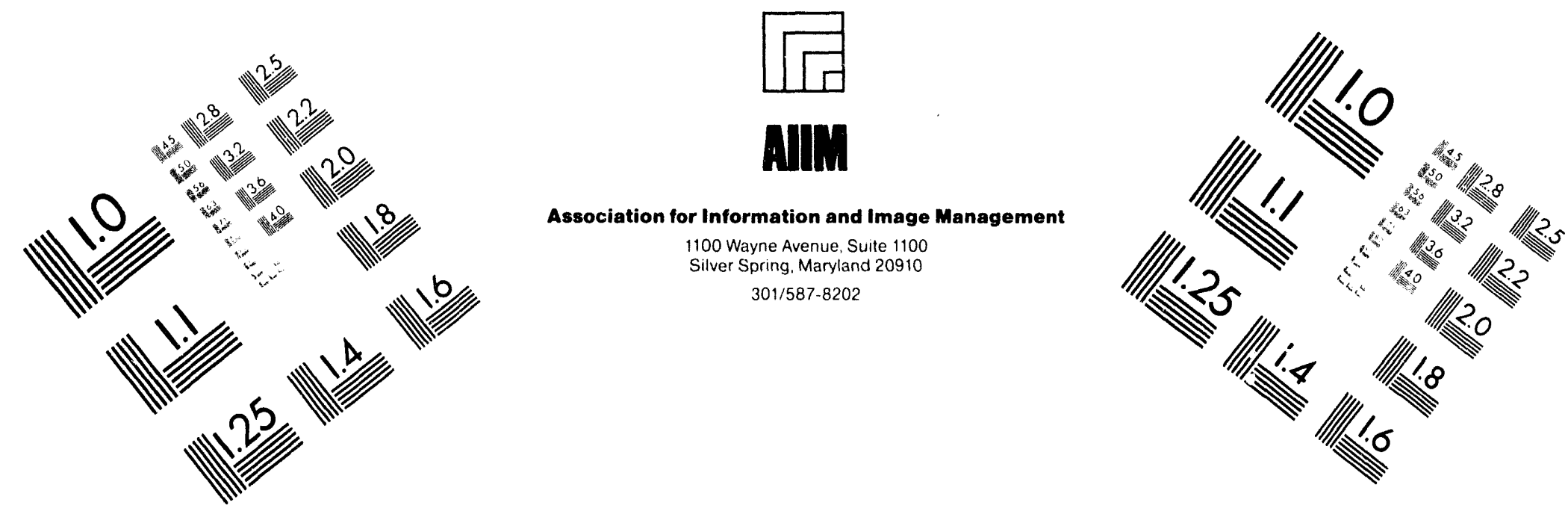

\section{Centimeter}

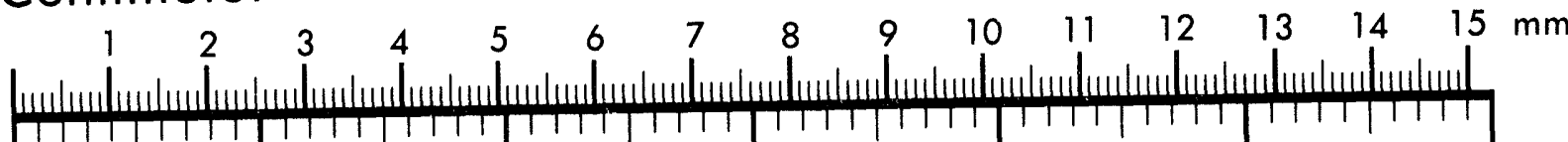

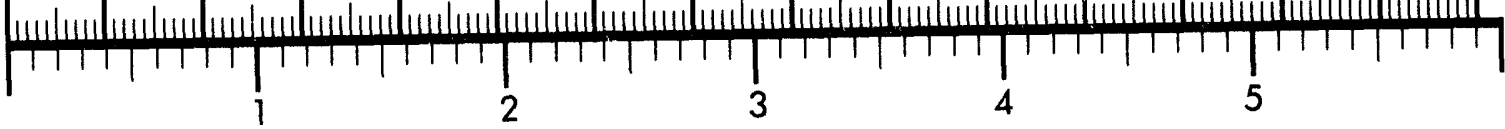
Inches
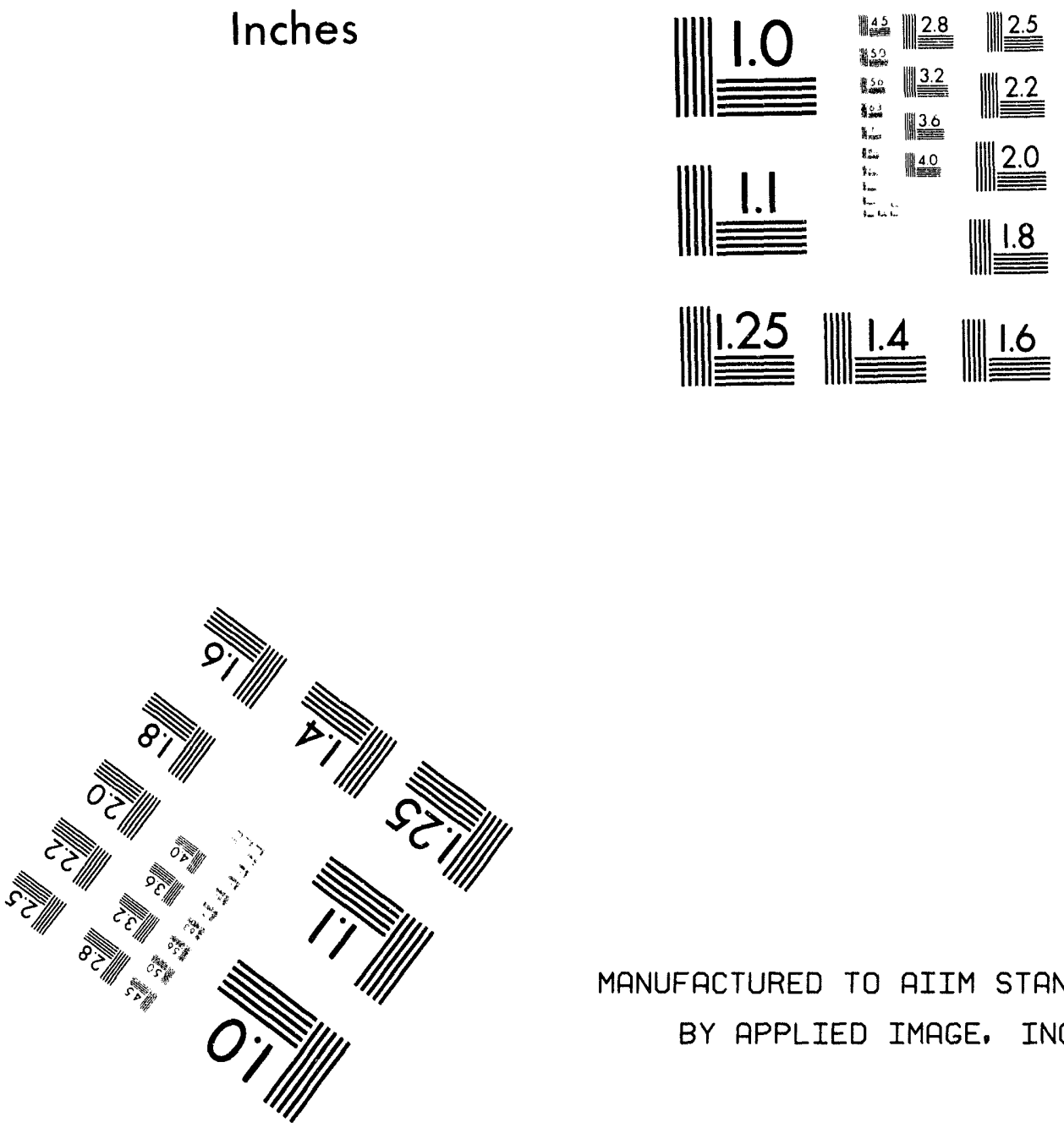

MANUFACTURED TO AIIM STANDARDS BY APPLIED IMAGE, INC.

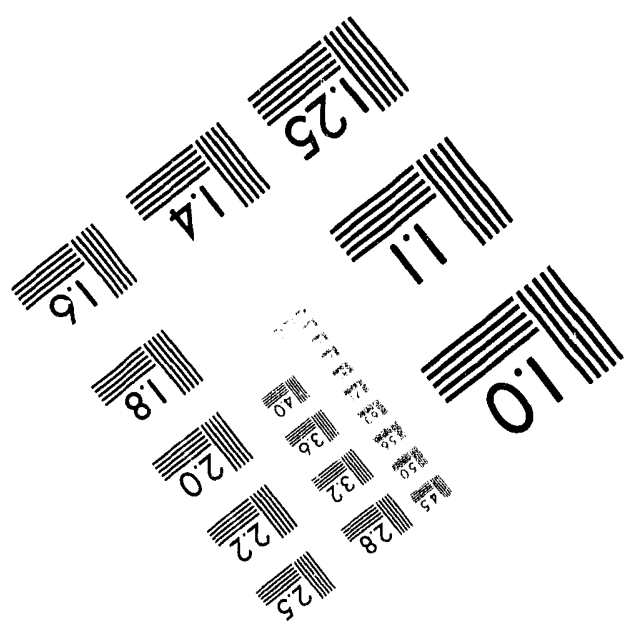


$E$
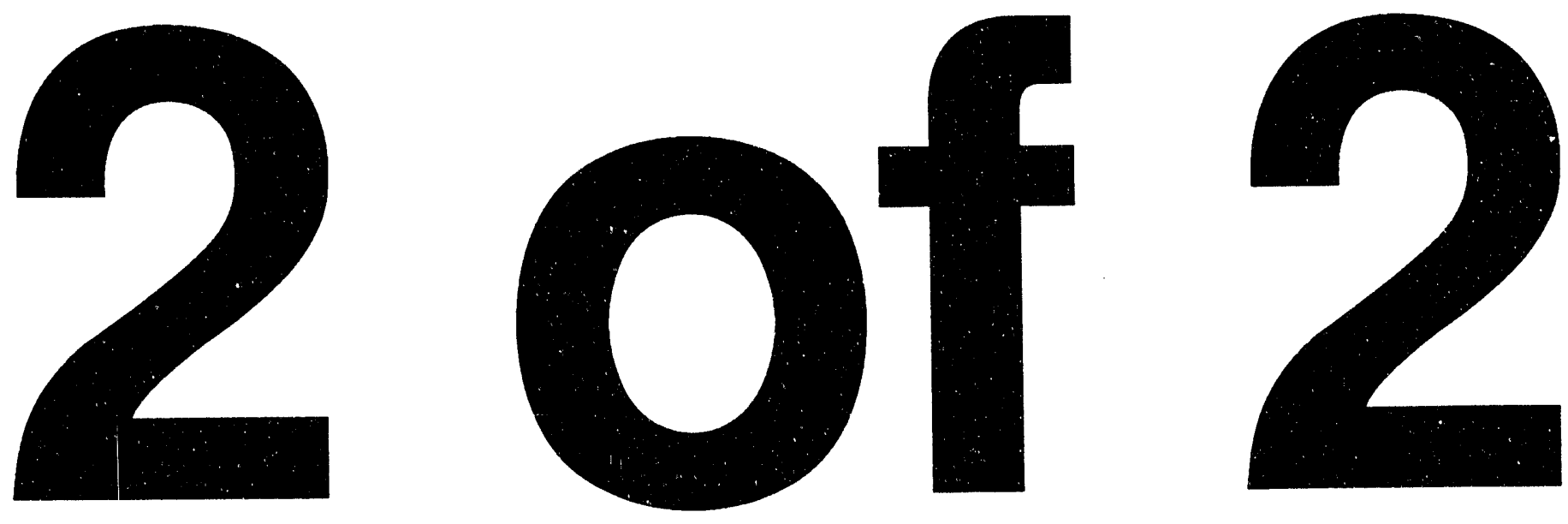


\section{Laingmuir Probe Pinout}

10:

NAME: LANGMUIR PROBES

NOTES:

PIN FUINTTION

28 VDC

-E* PROBE SIGNAL

- PROBE SIGNAL

-E- RANGE MONITOR

$\because$ RANGE MONITOR

-E" SWEEP MONITOR

$\because$ " SWEEP MONITOR

BOOM DEPLOYMENT MONITOR

GUN PULSE SYNC

PREGUN PULSE SYNC

DATA SYNC (2KHZ)

DATA SYNC RESET $(500 \mathrm{HZ})$

BOOM SWITCHING MONITOR

$\mathrm{N} / \mathrm{C}$

$\mathrm{N} / \mathrm{C}$

TO BOOM SWITCH *1

$\mathrm{N} / \mathrm{C}$

28 VOC RETURN

"E" PROBE SIGNAL RETURN

$" \because "$ FROBE SIGNAL RETURN

"E" RANGE MONITOR RETURN

"I" RANGE MONITOR RETURN

"E" SWEEP MONITOR RETURN

"I" SWEEP MONITOP RETURN

EOOM DEPLOYMENT MONITOR RETURN

GUN PULSE SYNC RETURN

PREGUN PULSE SYNC RETURN

DATA SYNC RETURN

DATA SYNC RESET RETURN

BOOM SWITCHING MONITOR RETURN

$N / C$

$N / C$

FROM BOOM SWITCH * 4

SHIELD

SHIELO

SHIELD

SHIELD

SHIELD

SHIELD

SHIELD

SHIELD

SHIELD

SHIIELD

SHIELD

SHIELD

SHIELD

WI

$1,:=$
CONN: DDM-5OP

MATE: DDM-50S

MFGR: CANNON

PINS: $\mathbf{5 0}$

NOTES

TWPR 1

TWPR 2

TWDR 3

TWPR 4

TWPR 5

TWPR 6

TWFR 7

TWPP 8

TWPR 9

TWPR 10

TWPR 11

TWPR 12

TWPR 13

CO-AX CABLE CONDUCTOR

TWPR 1

TWPR 2

TWPR 3

TWPR 4

TWPR 5

TWPR 6

TWPR 7

TWPR 8

TWPR 9

TWPR 10

TWPR 11

TWPR 12

TWPR 13

CO-AX CABLE CONDUCTOR

TWPR 1

TWPR 2

TWPR 3

TWPR 4

TWPR 5

TWPR 6

TWPR 7

TWPR 8

TWPR 9

TWPR 10

TWPR 11

TWPR 12

TWPP 13

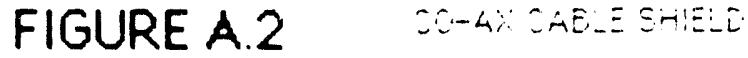




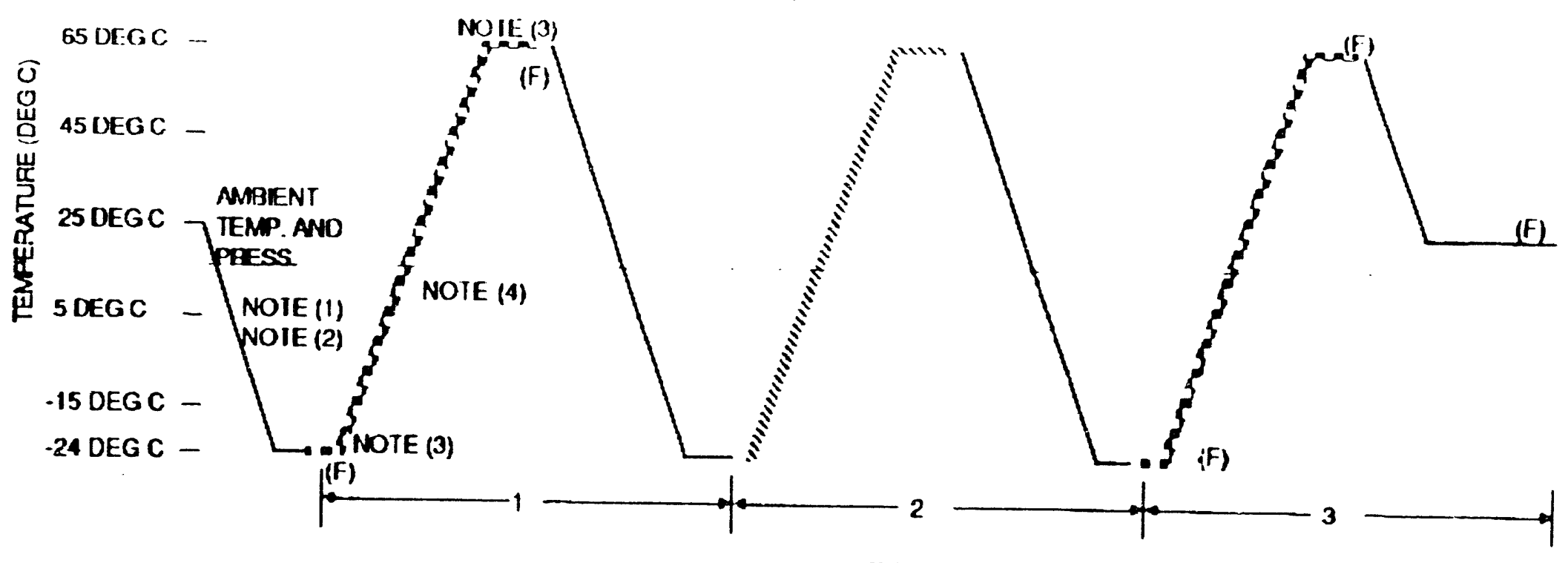

TIEPML CYCIES

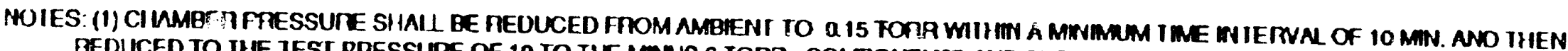
PREDUCED TO IHE TEST PRESSURE OF 10 TO TIE MINUS 6 TORR COMPONENIS AND SUBSYSTEMS TILT ANE ORERAIMG AT LAUNCH

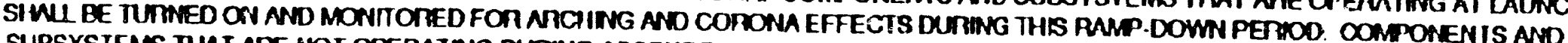
SUPSYSIEMS THAT ARE NOT OPERATING DURIIG ASCENT TO THE IEST ALIIIUDE WUL BE TURNED OFF UNIL THE IEST PRESSURE
IS REACIIED.

(2) WITH TIEE IEST ARTKCLE TURNED OFF. THE IEST ARTICLE IEMPERATURE WIL BE REDUCED FPOM AMBIENT TO -24 DEG C AT A PATE OF AT LEAST 3 DEG C PER MMN. (3) WIERMML STABMIZAIION PE? HOD (STABIIZAIION IS REACHED WIEN THE TEMPERATUAE RATE OF CHANGE IS LESS IHAN OREOUAL TO
2 DEG C. PEA HOUT).

(4) THERMN RUMP UP AND DOWN SHALL BE AT LEAST 3 DEG C PER MNN.

(F) FUNCIKONAL TEST

2 DURING THE FIRST AND LAST CYCLES, AFIER LOW IEMI'CMUIURE SIABILLAIION. THE UNIT WILL BE IUPNED ON ANO OPERATED FON IIIOUR.

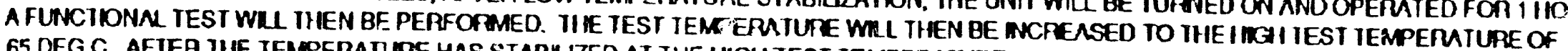
65 DEG C. AFIEA IHE TEMPERATURE HAS STABHIZED AT THE HIGI TEST IEMPEAAIURE, A FUNCIONAL IEST WML BE PERFORMED. IAS SIABILIZED AT AMIBIENT TEMPERATUFE.

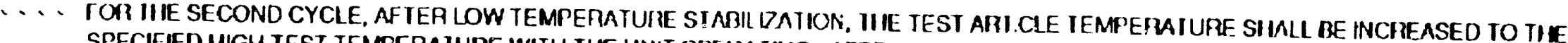
SPECIFIED HGH I TEST IEMPERAIURE WITH THE UNIT OPEHA TING. AFTEA IHE TEMPERATURE HAS SIABR RED AT THE HIGH IEST IEMPERATURE, THE UNII WILL BE IUTANED OFF.

FIGURE A.3 


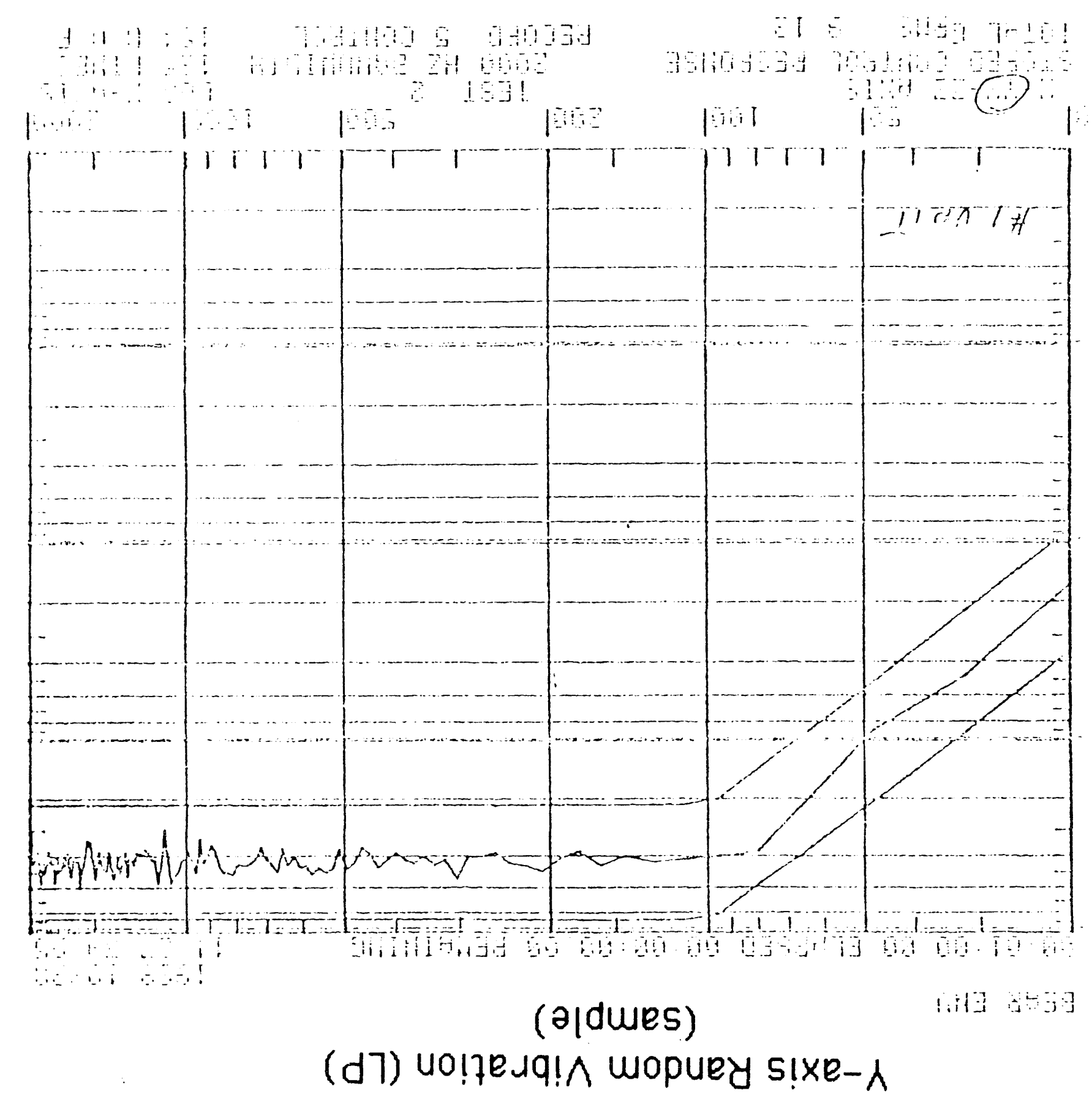


$N I \backslash N D$

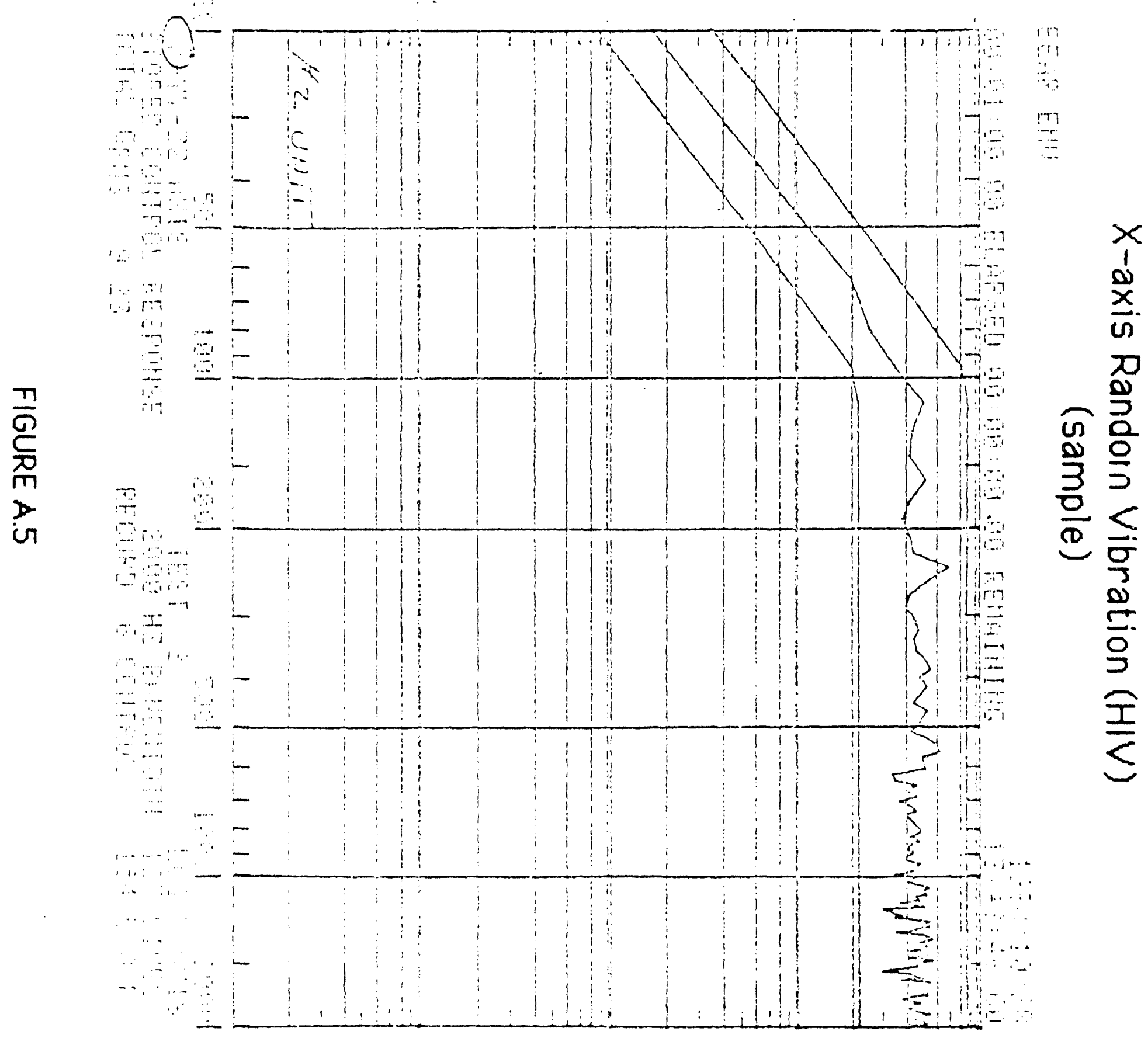




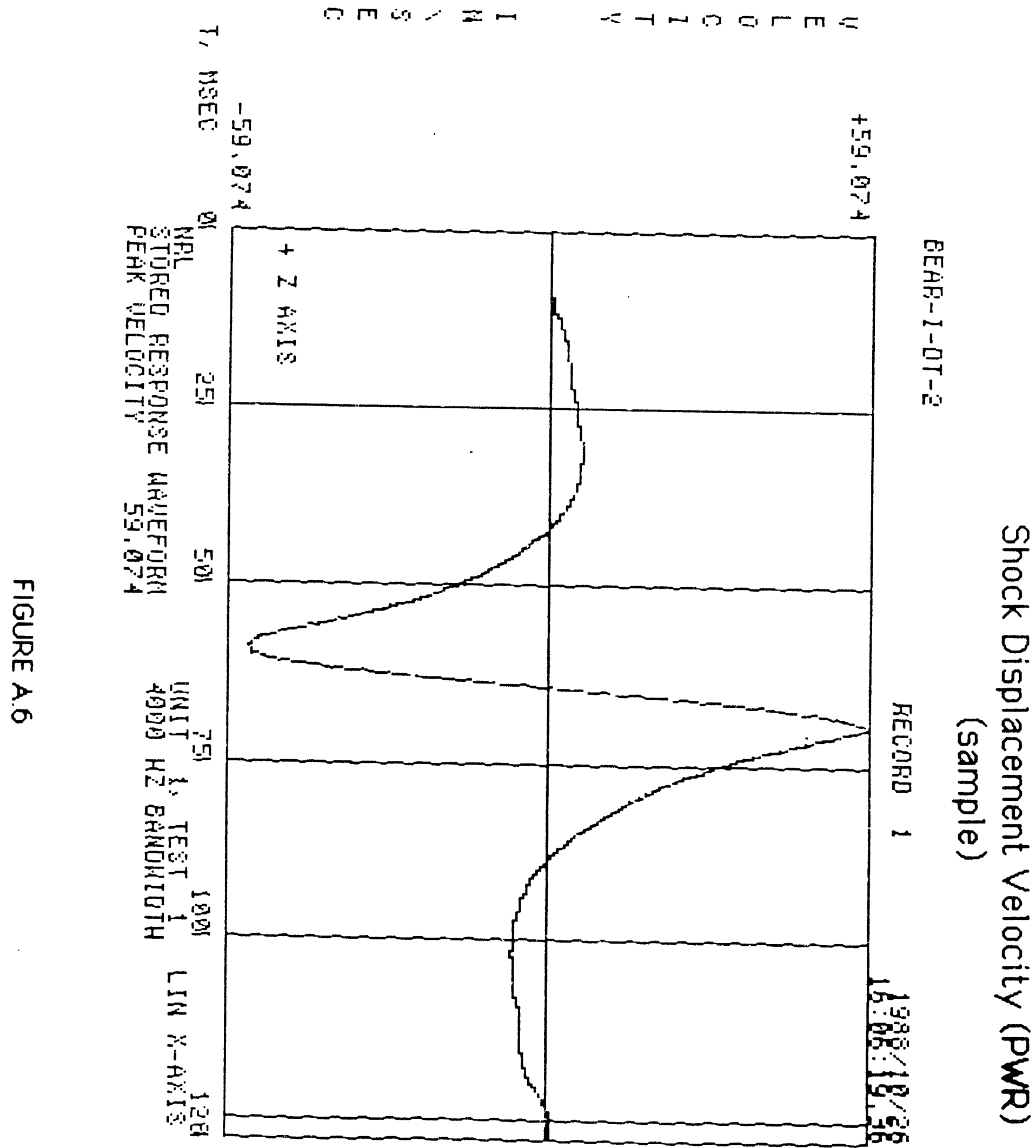




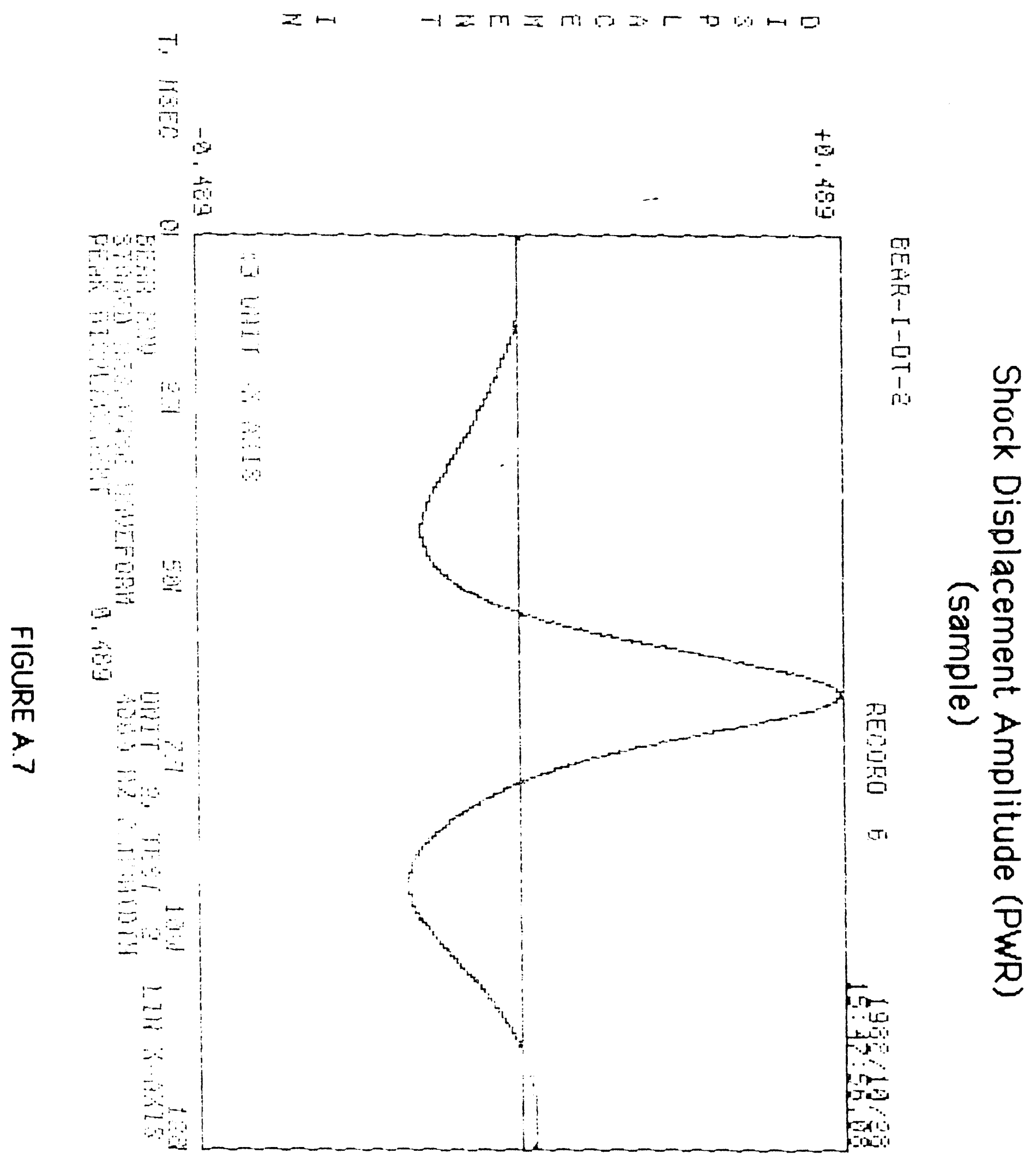



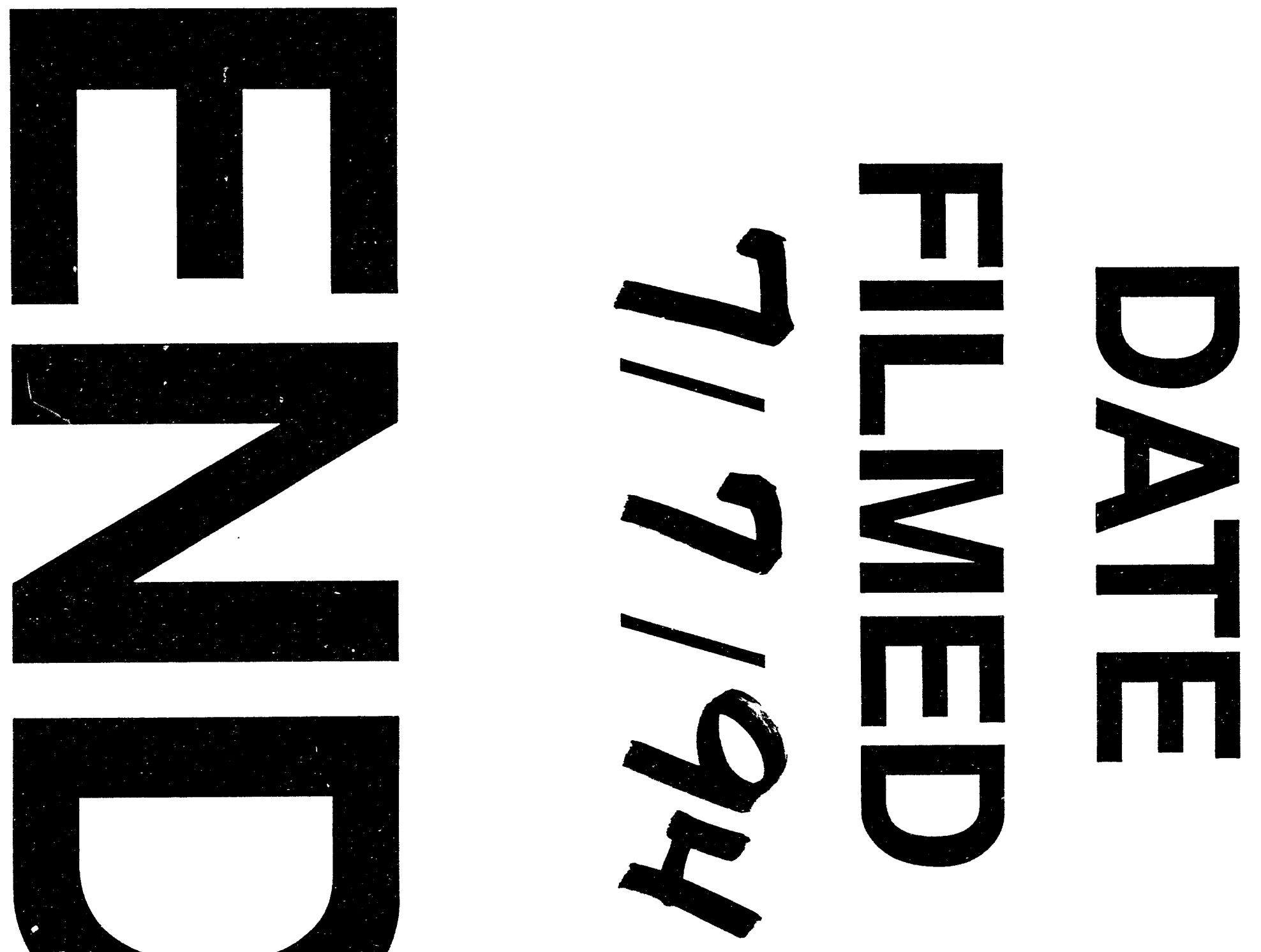

$\Sigma$

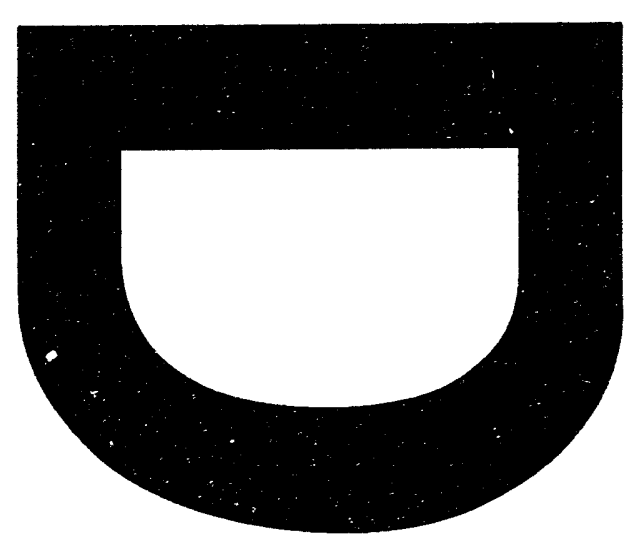


